\title{
Analysis of factors that influence the ICT adoption by SMEs in Colombia
}

\author{
Carlos Andrés Osorio-Gallego(iD), John Hildebrando Londoño-Metaute (iD), Esteban López-Zapata (iD) \\ Universidad de Antioquia (Colombia) \\ caosoga@gmail.com,jobn.londono@gmail.com,esteban.lopez@udea.edu.co
}

\section{Abstract}

Purpose: The objective of this article is to analyze the factors that influence the adoption of Information and Communication Technologies (ICT) by SMEs in Colombia.

Design/methodology: To prove empirically these hypotheses, we administered a questionnaire to a sample of 474 SMEs in Colombia regarding the adoption of ICT and independent variables identified in the literature. Then, we conducted a series of multiple regression models using the data we obtained.

Findings: We developed a model that identifies a positive relation between new business opportunities that ICT offer and their adoption level. Likewise, this research identified that a lack of confidence in ICT's security and privacy, a perception of ICT cost-benefit unbalance have a negative impact on the implementation of these technologies.

Research limitations/implications: This study has limitations that are inherent to crosssectional research. These restrict the possibility of conducting dynamic analysis between independent and dependent variables. Likewise, the generalization of results is circumscribed to a geographical context and the type of enterprises surveyed. 
Practical implications: The results of this research provide valuable contributions to decisionmaking by SMEs' directors and to public policies that foster the implementation of ICT in SMEs based on an analysis of benefits and barriers found in this study.

Originality/value: In view of a lack of empirical work that analyzes the implementation of technology in enterprises in emerging countries, this study provides a valuable contribution to the creation of knowledge when attempting to explain the implementation of ICT using a representative sample of SMEs in Colombia identifying the quantitative form of the impact of the benefits and barriers of these technologies.

Keywords: Technological adoption, Diffusion of innovation, ICT, SMEs, Colombia

Jel Codes: O32, O33, M15

\section{Introduction}

Information and Communication Technologies (ICT), and specially, the use of Internet and Web services are changing rapidly the traditional way for companies to do business. With the progressive globalization of economy, ICT are among the most versatile tools to allow small and medium enterprises to compete profitably in larger and more dynamic markets.

A continuous growing interest in the ICT adoption is also attributed to the exponential growth of the number of internet users worldwide, and a greater increase of users reported in developing countries when you compare Latin America and the United States of America, Europe and Asia. In fact, currently, 3.2 billion people are using internet, and of these, 2 billion are from developing countries (ITU, 2015).

Even though the countries with a higher GDP per capita, literacy rates, well-established telecommunications infrastructures and political stability have greater internet diffusion, the gap with developing countries has begun to decrease. First of all, internet penetration rates have begun to decrease in the former and have begun to grow in the latter. Second, this is because new mobile broadband technology has become more accessible to countries in via of development (ITU, 2015). 
For instance, in countries like Colombia, broadband Internet Connections have been growing at a rate of 20.4\% inter-annually and Internet penetration in December 2014 was 22.3\% (10.6 millions of users) (Ministerio de TIC, 2015). This equals approximately the fifth part of the population in comparison with 4.4 million users in 2010 or 874 thousand users in 2000 (Orduz, 2011).

The government of Colombia has been conducting a digital plan named 2010-2014 Live Digitally plan (in Spanish, Plan Vive Digital 2010 - 2014) which is focused on large-scale internet access. For instance, with a project named, National Fiber Optic Network (Red Nacional de Fibra Óptica) the number of townships connected multiplied 5 times using fiber optic networks, and auctioning of the radio spectrum has allowed $4 \mathrm{G}$ coverage in 50\% of the country (Ministerio de TIC, 2014). Therefore, serious attention is perceived in the development of telecommunications infrastructure and a context for ICT to guarantee that the country may advance rapidly into the information age.

Perhaps, the information technology that has penetrated SMEs most is Internet. In fact, just in 2010 alone, 60.6\% already used Internet to purchase supplies, make financial transactions and conduct marketing, etc. (Ministerio de TIC, 2014).

In Colombia, $99.9 \%$ of the companies are SMEs, this equals approximately 1.6 million corporate units, and just as it happens in other places, these companies concentrate $81 \%$ of all employees while their contribution to the GDP is just 37\% (Cámara Colombiana de Informática y Tecnología \& Fedesarollo, 2013). Even though the growing penetration of the use of Internet is quite clear in Colombian SMEs, it is necessary that they align these ICT with their business objectives and include technological tools as web 2.0 as essential alternatives in their activity or production plans (Cabarcas Álvarez, Martelo Gómez \& Puello Marrugo, 2013). It is important for SMEs to consider the use and adoption of ICT to reach a larger group of potential clients domestically and globally.

While several authors have identified various factors that affect the adoption or not of websites and ecommerce, there are still some doubts on how to approach this. Studies conducted up to now provide a narrow approach regarding SMEs, mainly, regarding the adoption of e-commerce.

There are theoretical models that have been used in previous studies to explain users' behavior when adopting technological innovations for instance the DOI - diffusion of innovations model, which has become popular in this field (Rogers, 1995; Tan, Chong, Lin \& Eze, 2009), or the Theory of Reasoned Action, the Theory of Planned Behavior and the Technology Acceptance Model. In some studies, these models are combined to construct new models (Benham \& Raymond, 1996; Limthongchai \& Speece, 2003; Tan \& Teo, 2000), and this has led to different results. 
Although previous studies have shared certain geographic proximity regarding the countries analyzed, we have identified that various studies that use the DOI model have rendered contradictory results (Hussin \& Noor, 2005; Kendall, Tung, Chua, Ng \& Tan, 2001; Limthongchai \& Speece, 2003). Furthermore, there is a scarcity of research combining DOI model variables and other constructs related to the benefits and barriers of ICT adoption.

Conducting a study of this type in a developing country as Colombia is a highly relevant factor. Despite the fact that the government has been investing actively in developing required infrastructure and that this has contributed to an increase in the number of internet users and the promotion of providing internet-based services, it is also true that the technological constraints that SMEs have been facing are mainly in part a lack of education and skills in ICT which is an important means to obtain a competitive advantage in this global market (Cámara Colombiana de Informática y Tecnología \& Fedesarollo, 2013).

Therefore, this study was conducted to answer the questions mentioned above. This study has as its objective to analyze the factors which influence the degree of ICT adoption by SMEs in Colombia.

In the context of this study, the adoption of ICT is understood as the definition proposed by Tan et al. (2009) regarding internet- based ICT. It considers the use of technologies as hardware, software and networks by SMEs to connect and use Internet. Although internet-based ICT are considered a new innovation, terminology is not the same like the one that was used by other authors when conducting technology- related studies (Chapman, James-Moore, Szczygiel \& Thompson, 2000; Hsieh, Jones \& Lin, 2008; Kumbakara, 2008; Lettieri \& Masella, 2005; Lucchetti \& Sterlacchini, 2004). Therefore, in this study, terminology for the adoption of ICT and the adoption of internet -based ICT is used indistinctively.

\section{Theoretical framework}

ICT have been approached from different contexts. The United Nations has considered ICT from a socio-economic approach. The International Telecommunications Union has defined ICT as an economic sector, and the United States and other countries have strongly related the use of ICT to an educational context. However, from a business point of view, ICT are technologies used by people and organizations to process their information and for communication purposes (Zhang, Aikman \& Sun, 2008). 
Internet- based ICT Adoption refers to how a company uses Internet to innovate in business and the use of websites as a communication platform within the company and with stakeholders (Narayanasamy, Santhapparaj \& Eze, 2008; Tan et al., 2009).

Companies that have been late ICT adopters are characterized by a lesser degree of penetration in ICT. This means that the diffusion of ICT in SMEs follows Rogers' S-curve theory of the technology diffusion pattern (Lucchetti \& Sterlacchini, 2004). Hepworth and Ryan (2000) argue that SMEs follow a pattern to normalize the diffusion of ICT, so ICT development gives credence to the idea that Internet is a platform that enables the growth of SMEs.

Regarding other studies conducted on the ICT adoption in SMEs, Bayo-Moriones and Lera-López (2007) have centered their attention on characteristics that identify the key determinants of the ICT adoption in Spanish companies. They paid special attention to variables like workers' ages, competitive pressure, international markets, competitive strategies or differentiation and ISO certification, amongst others.

On the other hand, while reviewing the literature of the various studies on the ICT adoption in SMEs, which have been conducted in other countries different from Colombia, we found that several conceptual and theoretical frameworks have been used regarding technological adoption. Nevertheless, according to Rouibah, Khalil and Hassanien (2009), there are basically 4 theories which have been widely used to explain technological adoption:

- TAM - Technological Acceptance Model (Davis, 1989);

- $\quad$ TRA - Theory of Reasoned Action (Ajzen \& Fishbein, 1980);

- $\quad$ TPB - Theory of Planned Behavior (Ajzen, 1985);

- DOI - Diffusion Of Innovations (Rogers, 1995). These theories are briefly described in Table 1.

There are other less known technology adoption models like the Technology-Organization Environment (TOE) framework (Tornatzky, Fleischer \& Chakrabarti, 1990), the PERM - Perceived Electronic Readiness Model (Molla \& Licker, 2005) and the Unified Theory of Acceptance and Use of Technology - UTAUT (Venkatesh, Morris, Davis \& Davis, 2003) which aims to unify various known models of acceptance, diffusion and adoption of technology. Nevertheless, they have been used to a lesser extent to explain the adoption of ICT in organizations (see Table 1). 


\begin{tabular}{|c|c|c|}
\hline Authors (Year) & Model & Description of the model \\
\hline Davis (1989) & $\begin{array}{l}\text { TAM - Technology Acceptance } \\
\text { Model }\end{array}$ & $\begin{array}{l}\text { When a user is presented with new technology, the "perceived } \\
\text { usefulness" and "the perceived ease of use" notably influence } \\
\text { how and when users utilize it. }\end{array}$ \\
\hline Ajzen and Fishbein (1980) & $\begin{array}{l}\text { TRA - Theory of Reasoned } \\
\text { Action }\end{array}$ & $\begin{array}{l}\text { This theory aims to predict individuals' behavior and attitudes. } \\
\text { The components of TRA are "behavioral intention", } \\
\text { "attitude" and "subjective norm". }\end{array}$ \\
\hline Ajzen (1985) & $\begin{array}{l}\text { TPB - Theory of Planned } \\
\text { Behavior }\end{array}$ & $\begin{array}{l}\text { This is a psychological theory that links individuals' beliefs and } \\
\text { behavior. To the three components the theory of reasoned } \\
\text { action, you add the "perceived behavioral control" construct. } \\
\text { The four constructs determine an individual's behavioral } \\
\text { intentions. }\end{array}$ \\
\hline Rogers (1995) & DOI - Diffusion of Innovation) & $\begin{array}{l}\text { The theory of Diffusion of Innovation indicates that early } \\
\text { adopters evaluate innovation for its relative advantage, } \\
\text { compatibility, complexity, trialability and observability. }\end{array}$ \\
\hline Tornatzky et al. (1990) & $\begin{array}{l}\text { TOE - Technology Organization } \\
\text { Environment Framework }\end{array}$ & $\begin{array}{l}\text { A company adopts and implements technological innovations } \\
\text { influenced by technological context variables (internal and } \\
\text { external technologies), organizational context variables (a } \\
\text { firm's characteristics and resources) and environmental } \\
\text { context variables (size and structure of a company) }\end{array}$ \\
\hline Molla and Licker (2005) & $\begin{array}{l}\text { PERM - Perceived eReadiness } \\
\text { Model }\end{array}$ & $\begin{array}{l}\text { The adoption of e-commerce in organizations depends on two } \\
\text { dimensions: POER -Perceived Organizational e-Readiness and } \\
\text { PEER- Perceived External e-Readiness. The constructs of } \\
\text { POER are conscientiousness, resources and commitment. The } \\
\text { constructs of PEER are forces including the market, } \\
\text { government and supporting industries. }\end{array}$ \\
\hline Venkatesh et al. (2003) & $\begin{array}{l}\text { UTAUT - Unified Theory of } \\
\text { Acceptance and Use of Technology }\end{array}$ & $\begin{array}{l}\text { The acceptance of the technology model which aims to } \\
\text { explain our users intention to use an information system and } \\
\text { the user's subsequent behavior based on } 3 \text { determinants of the } \\
\text { intention to use (performance expectation, effort expectation } \\
\text { and social influence) and a determinant of the behavior of use } \\
\text { (facilitating conditions) }\end{array}$ \\
\hline $\begin{array}{l}\text { Bayo-Moriones and Lera- } \\
\text { López (2007); Spanos, } \\
\text { Prastacos and } \\
\text { Poulymenakou (2002) }\end{array}$ & $\begin{array}{l}\text { Adoption of ICT at a } \\
\text { corporate level }\end{array}$ & $\begin{array}{l}\text { The adoption of ICT in companies depends on environment, } \\
\text { human capital, internal organization, strategy and a firm's } \\
\text { characteristics }\end{array}$ \\
\hline
\end{tabular}

Table 1. Technological Adoption Models

To conduct this study on the adoption of ICT in SMEs in Colombia, we did not use the TMA because it does not completely reflect social influences on an organizational context (Alkalbani, Rezgui, Vorakulpipat \& Wilson, 2013), but instead it seeks to explain and individual's intention to develop the behavior at issue (Grandon \& Mykytyn Jr, 2004). In addition, we did not use the TRA model because it is mostly oriented to predicting the fulfillment of objectives (Riemenschneider \& McKinney, 2001). Likewise, we did not use the TPB model because it is mostly focused on explaining and predicting managers' behavioral intentions and not on the adoption of technology at an organizational level (Mykytyn Jr \& Harrison, 1993). 
Of the above models for technological adoption, the DOI model (Rogers, 1995) is one of the most used to explain technological adoption in organizations, understood as a type of diffusion of innovation within companies (Brancheau \& Wetherbe, 1990; Hussin \& Noor, 2005; Kendall et al., 2001; Syed, Khatibi, Ismail \& Ahmad, 2005; Teo \& Pok, 2003; Van Slyke, Belanger \& Comunale, 2004). Some studies have even combined the constructs of the DOI model with other models like TRA, TPB and TAM (Benham \& Raymond, 1996; Limthongchai \& Speece, 2003; Tan \& Teo, 2000). Table 2 illustrates studies on the adoption of ICT in SMEs that have based themselves on the DOI model.

\begin{tabular}{|l|l|}
\hline \multicolumn{1}{|c|}{ Authors (year) } & \multicolumn{1}{c|}{ Results } \\
\hline Kendall et al. (2001) & $\begin{array}{l}\text { E-commerce was studied as a new innovation among SMEs in Singapore. Results indicate that relative } \\
\text { advantage, compatibility and trialability are factors that affect the adoption of e-commerce. } \\
\text { Observability and complexity are not significant. }\end{array}$ \\
\hline $\begin{array}{l}\text { Beatty, Shim y Jones } \\
\text { (2001) }\end{array}$ & $\begin{array}{l}\text { The factors that influence the adoption of corporate web sites were analyzed in 286 SMEs in the } \\
\text { United States. The independent variables were perceived benefits, complexity, compatibility and } \\
\text { managerial support. The most significant were perceived benefits (relative advantage) and complexity. }\end{array}$ \\
\hline $\begin{array}{l}\text { Limthongchai y } \\
\text { Speece (2003) }\end{array}$ & $\begin{array}{l}\text { The adoption of e-commerce was studied among owners, presidents and CEOs of SMEs in Thailand. } \\
\text { In addition to the five characteristics of DOI, they added security or confidentiality. Relative } \\
\text { advantage, compatibility, security or confidentiality and observability are positively correlated to the } \\
\text { adoption of e-commerce while complexity had a negative correlation. }\end{array}$ \\
\hline $\begin{array}{l}\text { Hussin y Noor } \\
(2005)\end{array}$ & $\begin{array}{l}\text { The position of SMEs in Thailand to adopt e-commerce was explored among CEOs. Relative } \\
\text { advantage, observability and complexity were significantly related to the adoption of e-commerce } \\
\text { while trialability and compatibility were not significant. }\end{array}$ \\
\hline Tan et al. (2009) & $\begin{array}{l}\text { The adoption of internet-based ICT in 406 SMEs in Malaysia was studied. Relative advantage, } \\
\text { compatibility, observability and security were significantly related in a positive way with the adoption } \\
\text { of ICT in SMEs while complexity had a negative association. }\end{array}$ \\
\hline $\begin{array}{l}\text { Moghaddam y } \\
\text { Khatoon-Abadi } \\
(2013)\end{array}$ & $\begin{array}{l}\text { Factors which affect the adoption of ICT by rural users in central Iran were studied. They proved that } \\
\text { DOI innovation characteristics had a significant positive effect on the adoption of ICT by those users } \\
\text { except for the compatibility variable. }\end{array}$ \\
\hline
\end{tabular}

Table 2. Previous studies on the adoption of ICT in SMEs based on the DOI model

In DOI, diffusion is understood as a social process by which an innovation is communicated in time via certain channels between the members of a social system (Rogers, 1995). On the other hand, innovation is defined as an idea, practice or object that is perceived as new by an individual unit or another type of adoption (Tan et al., 2009).

According to this model, there are five main influences in a user's behavior when accepting innovation: Relative advantage, complexity, compatibility, observability and trialability (He, Duan, Fu \& Li, 2006).

Rogers (1995) described each one of these key five influences or important characteristics perceived from innovation as follows: 
- Relative advantage - the degree in which innovation is perceived as being better than what it replaces.

- Compatibility - the degree in which innovation is coherent with existing values, past experiences and needs.

- Complexity - the degree in which innovation is difficult to understand and use.

- Trialability - the degree in which innovation can be submitted to experimentation or tests.

- Observability - the degree of visibility of the new results of innovation.

While it is true that many researchers have used the DOI model with its five characteristics of innovation, it is also true that many studies have proven that characteristics as compatibility and trialability are not usually significant when explaining the adoption of ICT in SMEs (Hussin \& Noor, 2005; Moghaddam \& Khatoon-Abadi, 2013; Ntemana \& Olatokun, 2012; Tan et al., 2009). Due to the above reasons, for this study regarding the adoption of ICT in SMEs in Colombia, just three of the five characteristics of DOI innovation were used (relative advantage, observability and complexity) as a reference framework for the adoption of ICT in SMEs, and the model was complemented with some constructs of benefits and barriers of the adoption of ICT.

\subsection{Relative Advantage}

Relative advantage is understood as "that degree in which innovation is perceived better than its predecessor" (Moore \& Benbasat, 1991). That is, that according to the theory of diffusion of innovation when adopting a new innovation (in this case a new ICT), potential adopters (in this case SMEs) evaluate the relative advantage it has in terms of perceived efficiencies of that innovation regarding current technologies or procedures (Rogers, 1995).

Relative advantage is seen as an important condition which influences the decision of adopting a technology, and it is considered as essential indicator for the adoption of new information systems (Alshamaila, Papagiannidis \& Li, 2013). The impact of relative advantage over technology adoption has been widely researched in previous studies (Gibbs \& Kraemer, 2004; Iacovou, Benbasat \& Dexter, 1995; Lee, 2004; Premkumar \& King, 1994; Ramdani \& Kawalek, 2007; Thong, 1999). It has been 
demonstrated that when businesses perceive a relative advantage in an innovation; then, the probability of the adoption will increase (Lee, 2004; Thong, Yap \& Raman, 1994; Thong, 1999).

Relative advantage is a function of the perceived usefulness of new or existing technologies which allows the incorporation of the influence of other technologies which would otherwise be ignored in an organization (Wang, Meister \& Wang, 2008). The decision of rational adoption in an organization requires the assessment of the possible benefits of a new technology for the business.

ICT represent a variety of earnings for companies which adopt them. For instance, according to Miller (2008) cloud computation (a type of ICT) may offer many advantages related to capacity, reliability and flexibility. This technology reduces considerably the entry costs of SMEs to access a large number of computational resources in relatively short terms of time. This allows small businesses to concentrate on the market without having to invest capital on hardware (Marston, Li, Bandyopadhyay, Zhang \& Ghalsasi, 2011). Likewise, Lin and Lin (2008) state that web technology is more likely to be adopted when organizations perceive that it will help with their businesses' information exchange. Therefore, from a perspective of ICT capacities, the relative advantages of these types of business technologies are considered a fact (Sokolov, 2009).

Then, in general terms, the adoption of ICT must be influenced positively by a relative advantage. Therefore, the following hypothesis is proposed:

H1. There is a positive relation between the adoption of ICT in SMEs and the relative advantage of those technologies.

\subsection{Observability}

Observability is defined as the degree in which the results of an innovation are visible to others. Most innovations studied in the diffusion of innovation research were technological topics (Rogers, 1995). Observability is difficult to establish in a market that is already dominated by an established technology. Therefore, it will be important to establish observability on a market niche or a new technological application before attempting to challenge the dominant technology in its market and its native application (Weiss \& Dale, 1998).

Currently, the observation of ICT like e-commerce is relatively easy for consumers and adopter industries. E-commerce increases a company's visibility because a website can be seen as a place to 
purchase. Websites allow businesses to stay open $24 / 7$ for millions of clients and potential suppliers on the Internet (Blackwood, 1997).

With technology like Internet, clients and suppliers are able to visit an SME's website look for general information and they will obtain a fast reply via e-mail or another medium anytime and anywhere. This creates convenience and flexibility so that SMEs may establish relations with sellers and purchasers.

If SMEs are able to see the benefits of the adoption of ICT, it is quite probable that they will adopt those technologies. According to Slegers, Singh and Hall (1998), one of the biggest barriers to access internet services is a lack of conscientiousness, and this leads us to formulate the following hypothesis:

H2. There is a positive relation between the adoption of ICT in SMEs and a visibility perceived using those technologies.

\subsection{Complexity}

Rogers (1995) defines complexity as the "degree in which an innovation is perceived as difficult to use". Adopting new technologies make SMEs face challenges in terms of a change in the processes in which they interact to conduct their business. New technologies may be friendly and easy to use to increase adoption rates (Parisot, 1995; Sahin, 2006).

Some authors suggest that new ideas which are easy to understand are more quickly adopted than innovations which require the adopter to develop new knowledge, skills and comprehension (Premkumar, Ramamurthy \& Nilakanta, 1994). Likewise, systems perceived as the easiest to use and the least complex have a greater possibility of being accepted and used by potential users (Agarwal \& Prasad, 1997).

It has been found in many studies that complexity is a significant factor in the decision to adopt (Chaudhury \& Bharati, 2008; Harindranath, Dyerson \& Barnes, 2008; Tiwana \& Bush, 2007). Unlike the other characteristics of DOI, this factor is negatively associated to the probability of adoption (the more complex technology is, the lower its probability of adoption). 
According to the review of literature regarding complexity, we are able to formulate the following hypothesis:

H3. There is a negative relation between the adoption of ICT in SMEs and the complexity to implement or use those technologies.

\subsection{New Business Opportunities (Benefits)}

Organizations must recognize that the adoption of innovation, either offers solutions to existing problems or presents new production opportunities including an increase in productivity and better operating efficiency (Zhu \& Kraemer, 2005). Organizations adopt a new technology when they see a need for that technology believing that it will take advantage of a business opportunity or close a gap in organizational performance (Mndzebele, 2013).

Likewise, there are business opportunities and potential benefits when SMEs implement technologies like e-commerce. Among the opportunities they obtain is the strengthening of their relation with clients, reaching new markets, upgrading business processes, reducing costs, improving business knowledge, attracting investors and creating new products and services. Also, technology represents an opportunity for SMEs to compensate additional weaknesses in fields like access to new markets and collecting and disclosing information at an international level, and this improves communication and creates greater employment flexibility (Scupola, 2001).

Technology advances at a very fast rate that undoubtedly it offers SMEs vast opportunities to access international markets and participate in the global supply chain if they adopt ICT (Fillis, Johannson \& Wagner, 2004). In order to do this, SMEs owners or managers must equip themselves with enough knowledge in this field.

According to what is stated above, undoubtedly, globalization and fast technological changes including the use of Internet and e-commerce can bring SMEs new business opportunities (Scupola, 2001). In this sense, Tan and Eze (2008) found in a study that ICT adoption among SMEs provides new business opportunities and access to information and market knowledge. Therefore, we can establish the following hypothesis:

H4. There is a positive relation between the adoption of ICT in SMEs and their potential to generate new business opportunities. 


\subsection{Effective Communication with Clients (Benefits)}

Narayanasamy et al. (2008) mention that an organization has adopted ICT internet websites as a communication platform in organizations and their stakeholders. Moreover, if the website contains an e-commerce platform, its interface may virtually enable any type of client order.

Some authors indicate that the adoption of ICT can contribute to a quality strategy in organizations. For instance, Spanos et al. (2002) indicate that ICT allow companies to reach a differentiating advantage to assure client relations via better quality and more capability to respond quickly to market changes.

Accordingly, Tan et al. (2009) found that the adoption of ICT in SMEs becomes an effective communication tool with clients. Therefore, we are able to establish the following hypothesis:

H5. There is a positive relation between the adoption of ICT in SMEs and an increase in effective client communication.

\subsection{Business Cost Reduction (Benefits)}

There is a belief that technologies like e-commerce contribute to the advance of businesses in developing countries. This is punctuated by the potential internet and communication technologies have to reduce transaction costs pushing to one side middlemen and facilitating links to global supply chains (Hempel \& Kwong, 2001; Molla \& Licker, 2005).

Also, according to Tan et al. (2009), the adoption of internet-based ICT offers benefits for SMEs in terms of cost reduction, for instance mailing costs.

The arguments above allow the formulation of the following hypothesis:

H6.There is a positive relation between the adoption of ICT in SMEs and cost reduction for SMEs.

\subsection{Government Incentives (Benefits)}

Based on the review of several authors' literature, Consoli (2012) mentions that public policies constitute a determining factor for the adoption of ICT in SMEs. In fact, governments play an important role promoting the adoption of ICT in countries. Good network infrastructure, healthy competition between network suppliers, easy access for loans, schemas and application programs are some of the initiatives that governments can adopt to encourage the use of ICT (Tan \& Eze, 2008). 
Therefore, recently, there has been a dramatic increase in the number of business-solution companies in Thailand as a consequence of the promotion of e-commerce by that country's government (Limthongchai \& Speece, 2003).

Yap, Thong and Raman (1994) assure that if the participation in a government computerization program does not result in more effective information systems, government incentives do encourage SMEs, which lack financial and technical resources, to systematize operations. Providing subsidies, low interest loans, seminars and technical experience can compensate SMEs' lack of resources and internal expertise. Many small businesses would not have systematized and would not have been successful if it had not been for those incentives.

The positive impact of ICT on productivity has been widely demonstrated and one of governments' main action lines in the search for better competitiveness is the promotion of these technologies in organizations (Bayo-Moriones \& Lera-López, 2007).

The argument above leads us to formulate the following hypothesis:

H7. There is a positive relation between the adoption of ICT in SMEs and government incentives to courage the adoption of ICT.

\subsection{Inadequate ICT for Business (Barriers)}

Among the barriers identified for the adoption of ICT in SMEs is the lack of suitability of ICT for business (Tan et al., 2009; Tan \& Eze, 2008).

In a study conducted by ECTEL (2009), the study identified that businesses cannot see the relevance of the use of Internet for transactions if they believe that their products and services are not suitable for online commerce. The lack of information and knowledge regarding available ICT solutions can also contribute to this perceived constraint. In fact, OECD (2002) has mentioned that the main reason that businesses give for not committing to e-commerce is that it is not suitable for the nature of their business.

At the same time in Colombia, according to the Colombian chamber of informatics and technology and Fedesarrollo (2013) (in Spanish, Cámara Colombiana de Informática y Tecnología \& Fedesarrollo), $83.6 \%$ of small companies in this country have realized that the main reason for not using Internet is that it is not needed for their business. 
The above leads us to propose the following hypothesis:

H8.There is a negative relation between the adoption of ICT in SMEs and the scarce adjustment of ICT for business.

\subsection{A Lack of Trust in ICT Security (barriers)}

Many studies have identified that ICT security and confidentiality are significant factors that influence the adoption of ICT (Tan et al., 2009). For instance, Aljifri, Pons and Collins (2003) identified several aspects that impact the level of reliability on e-commerce, and since this affects the possibility of doing business online between companies, they considered that the security of information was one of the main barriers that affects e-commerce in developing countries.

Likewise, via assessments of SMEs' perception regarding inhibitors of the adoption of e-commerce, it has been found that one of the most influential factors was being concerned about security (APEC Asia Pacific Economic Cooperation, 1999).

Also, even though ICT application barriers in SMEs vary depending on the economic sector and on the country, there are common barriers for all companies including security and reliability factors (security and reliability of e-commerce systems, the uncertainty of online payment methods and legal frameworks)(Arendt, 2008).

On the other hand, in a survey, those SMEs, which answered that they did not adopt e-commerce, indicated that one of the main reasons was information security (Wymer \& Regan, 2005).

There are also other studies on the adoption of ICT in which they used security and privacy as an important dimension to evaluate, complementing the DOI model. Among these studies, some outstanding works included those by Tan and Teo (2000), Limthongchai and Speece (2003) and Hussin and Noor (2005).

Then, in general terms, the adoption of ICT in SMEs seems to be influenced negatively by security factors; therefore, we proposed the following hypothesis:

H9. There is a negative relation between the adoption of ICT in SMEs and the lack of confidence in ICT security and privacy. 


\subsection{High Investment in ICT with No Benefits in Return (Barriers)}

It's difficult to have a cost-benefit analysis of the impact of computational systems on organizations. Even though, Ives and Olson (1984) attempted to define the success of information systems as an added organizational benefit they produce in comparison with alternative investments, the truth is that several studies have failed to find evidence on the fact that the use of computers or related technologies improve the performance of SMEs (Cragg, 1990; Cron \& Sobol, 1983; Yap \& Walsham, 1986).

In many SMEs, ICT are not perceived as generators of value, and medium and small businesses believe that ICT costs do not cover the benefits they generate (Chesher \& Skok, 2000). Likewise, many SMEs see ICT costs as a barrier for the use and adoption of e-commerce and online business (Wymer \& Regan, 2005).

Also, Arendt (2008) found that even if the barriers for the implementation of ICT in SMEs vary and depending on an economic sector and the country, they are common for all companies including cost factors as ICT equipment and networks, software costs and reorganization and operating costs. Based on the above arguments, we formulate the following hypothesis:

H10. There is a negative relation between the adoption of ICT in SMEs and implementation cost unbalance in comparison with the benefits received from them.

The following section describes the methodology used in this study to contrast hypotheses.

\section{Methodology}

\subsection{Sample and Procedures}

To contrast hypotheses, we used a database taken from Confecámaras which has information on Colombian companies in the different economic sectors. An electronic questionnaire was designed and administered to a sample of 474 industry and services SMEs distributed as seen in Table 3.

\begin{tabular}{|c|c|r|}
\hline \multirow{2}{*}{ Economic Sector } & Services & $72 \%$ \\
\cline { 2 - 3 } & Industry & $28 \%$ \\
\hline \multirow{2}{*}{ Size } & Less than 10 employees & $65 \%$ \\
\cline { 2 - 3 } & 11 to 200 employees & $35 \%$ \\
\hline
\end{tabular}

Table 3. Characterization of participating companies 
The questionnaire was designed based on scales developed in previous studies; just like, we explain in the following section. First, a pilot test was developed including 20 SMEs directors in the city of Medellin, and we received feedback from some academic researchers to verify the content of the scales, minimize ambiguities and adapt the questionnaire's language to the context of Colombian SMEs. Then, the adjusted virtual questionnaire was administered, and the statistical processing of the information collected was conducted using a data analysis software named Stata version 11.2. Via a principal component factorial analysis, scale validity and reliability were verified; and then, an analysis of multiple regression models was conducted to contrast proposed hypotheses.

\section{Measures}

\subsection{Dependent Variable}

To measure the dependent variable, adoption of ICT, a scale was adapted based on Lucchetti and Sterlacchini (2004) and Bayo-Moriones and Lera-Lopez's (2007) studies. We use a 5-point scale regarding a company's use of a series of information and communication technologies (5: Currently use them, 4: Plan to use them next year, 3: Plan to use them after one year, 2: Does not plan to use them, 1: Does not know about them). The technologies taken into account are personal computers (workstations or computer terminals), company e-mail, LAN or WAN, internet, intranet, company webpages, company social networks and e-commerce.

To measure the adoption of ICT, a scale composed of all the previous technologies was considered, but in the regression models, the individual adoption of each one of these technologies was considered a dependent variable.

\subsection{Independent Variables}

The measurement scales for our independent variables were obtained from literature and from previously revised studies on the adoption of ICT in which the validity and suitability of ICT had already been proven. The 10 independent variables used in this study include 5 point Likert scales (5: totally agrees, 4: Agrees partially, 3: Neither agrees or disagrees, 2: Disagrees partially, 1: Totally disagrees). The chosen items to measure the constructs of relative advantage, observability and complexity were based on Ghobakhloo, Arias-Aranda and Benitez-Amado (2011) and Ntemana and 
Olatokun's (2012) studies. To do so, the items used by these authors were translated to Spanish, and based on the pilot test, the most representative comprehensible items were selected for the target public bearing in mind the constraints of the length of the questionnaire so that it would not affect interviewees' willingness to answer the questionnaire.

The items chosen to measure the constructs of benefits (new business opportunities, effective client communication, business cost reduction, and government incentives) and barriers (inappropriate ICT for the business, a lack of reliability in ICT security, and an ICT cost and benefit unbalance) were based on Thong and Yap (1995), Premkumar (2003), Grandon and Pearson (2004), Saffu, Walker and Hinson (2007) and Al-Qirim's (2006) studies. Just like with the above scales, they were translated to Spanish and the most representative and comprehensible items were selected based on the pilot test.

To guarantee scale reliability and validity, Cronbach's Alpha coefficient was analyzed for each construct, and a principal component factorial analysis (PCA) was conducted to assure their convergent validity and discriminant validity (Hair, Anderson, Tatham \& Black, 1999).

In Table 4, we present the items used on the scale reliability analysis. You can see that almost all the scales have a Cronbach's Alpha coefficient over 0.7, just as Nunnally and Bernstein (1994) recommended it. Even so, scales for complexity, effective client communication, government incentives and ICT cost and benefit unbalance presented values below 0.7. In those cases the items corresponding to variables COJ3, CEF3, IGO3 y DES3 were eliminated, and as a result of this, Cronbach's Alpha coefficients of the scales increase to $0.852,0.771,0.869$ and 0.708 respectively.

\begin{tabular}{|c|c|c|c|c|}
\hline Construct & $\begin{array}{l}\text { Variable } \\
\text { Code }\end{array}$ & Item & $\begin{array}{c}\text { Cronbach 's Alpha } \\
\text { coefficient } \\
\text { excluding the } \\
\text { variable }\end{array}$ & $\begin{array}{c}\text { Cronbach's } \\
\text { Alpha coefficient } \\
\text { of the scale }\end{array}$ \\
\hline \multirow{3}{*}{$\begin{array}{l}\text { Relative } \\
\text { advantage } \\
\text { (REL) }\end{array}$} & REL1 & $\begin{array}{l}\text { When we use ICT, efficiency improves in my } \\
\text { company }\end{array}$ & 0.830 & \multirow{3}{*}{0.855} \\
\hline & REL2 & $\begin{array}{l}\text { There are enough advantages in ICT to consider } \\
\text { using them in the company }\end{array}$ & 0.810 & \\
\hline & REL3 & ICT help manage company time better & 0.750 & \\
\hline \multirow{3}{*}{$\begin{array}{l}\text { Observability } \\
\text { (OBS) }\end{array}$} & OBS1 & $\begin{array}{l}\text { We were influenced by what we saw as ICT } \\
\text { benefits for the company }\end{array}$ & 0.749 & \multirow{3}{*}{0.784} \\
\hline & OBS2 & $\begin{array}{l}\text { We saw other companies using ICT, and we saw } \\
\text { the advantages of using them in the company }\end{array}$ & 0.624 & \\
\hline & OBS3 & $\begin{array}{l}\text { Before using ICT in the company, we saw how } \\
\text { other companies used them }\end{array}$ & 0.743 & \\
\hline
\end{tabular}




\begin{tabular}{|c|c|c|c|c|}
\hline Construct & $\begin{array}{l}\text { Variable } \\
\text { Code }\end{array}$ & Item & $\begin{array}{l}\text { Cronbach 's Alpha } \\
\text { coefficient } \\
\text { excluding the } \\
\text { variable }\end{array}$ & $\begin{array}{l}\text { Cronbach 's } \\
\text { Alpha coefficient } \\
\text { of the scale }\end{array}$ \\
\hline \multirow{3}{*}{$\begin{array}{l}\text { Complexity } \\
\text { (COJ) }\end{array}$} & COJ1 & $\begin{array}{l}\text { ICT are difficult to understand and use in the } \\
\text { company }\end{array}$ & 0.395 & \multirow{3}{*}{0.667} \\
\hline & COJ2 & ICT are confusing in the company & 0.350 & \\
\hline & $\operatorname{COJ} 3\left(^{* *}\right)$ & $\begin{array}{l}\text { It is not complicated to learn to use ICT in the } \\
\text { company (*) }\end{array}$ & 0.852 & \\
\hline \multirow{3}{*}{$\begin{array}{l}\text { New business } \\
\text { opportunities } \\
\text { (NOP) }\end{array}$} & NOP1 & $\begin{array}{l}\text { ICT have enabled the company to increase its } \\
\text { range of coverage and reach new markets }\end{array}$ & 0.783 & \multirow{3}{*}{0.860} \\
\hline & NOP2 & $\begin{array}{l}\text { ICT have allowed the company to enter new } \\
\text { business models }\end{array}$ & 0.797 & \\
\hline & NOP3 & $\begin{array}{l}\text { Using ICT, my company has been able to } \\
\text { connect with new suppliers }\end{array}$ & 0.831 & \\
\hline \multirow{3}{*}{$\begin{array}{l}\text { Effective client } \\
\text { communicatio } \\
\text { n (CEF) }\end{array}$} & CEF1 & $\begin{array}{l}\text { In the company, we do not use ICT to } \\
\text { communicate with clients }\left(^{*}\right)\end{array}$ & 0.500 & \multirow{3}{*}{0.669} \\
\hline & CEF2 & $\begin{array}{l}\text { We prefer to use traditional media instead of ICT } \\
\text { to communicate with clients }\left(^{*}\right)\end{array}$ & 0.396 & \\
\hline & CEF3 (**) & $\begin{array}{l}\text { Using ICT my company can communicate more effectively } \\
\text { with clients }\end{array}$ & 0.771 & \\
\hline \multirow{3}{*}{$\begin{array}{l}\text { Business Cost } \\
\text { Reduction } \\
\text { (RCO) }\end{array}$} & RCO1 & $\begin{array}{l}\text { Using ICT in my company we have reduced } \\
\text { operating costs }\end{array}$ & 0.873 & \multirow{3}{*}{0.848} \\
\hline & $\mathrm{RCO} 2$ & $\begin{array}{l}\text { After implementing ICT solutions in the } \\
\text { company, processes are faster }\end{array}$ & 0.703 & \\
\hline & $\mathrm{RCO} 3$ & $\begin{array}{l}\text { The company is more productive after } \\
\text { incorporating ICT into our key processes }\end{array}$ & 0.775 & \\
\hline \multirow{3}{*}{$\begin{array}{l}\text { Government } \\
\text { incentives } \\
\text { (IGO) }\end{array}$} & IGO1 & $\begin{array}{l}\text { In the company, we implemented ICT because of } \\
\text { a government support initiative }\end{array}$ & 0.132 & \multirow{3}{*}{0.544} \\
\hline & IGO2 & $\begin{array}{l}\text { If it had not been for government incentives, we } \\
\text { would not have adopted ICT in the company }\end{array}$ & 0.027 & \\
\hline & IGO3 (**) & $\begin{array}{l}\text { Government incentives are not effective in belping } \\
\text { companies to adopt ICT }\left(^{*}\right)\end{array}$ & 0.869 & \\
\hline \multirow{3}{*}{$\begin{array}{l}\text { Unsuitable } \\
\text { ICT for the } \\
\text { business (INA) }\end{array}$} & INA1 & $\begin{array}{l}\text { We worry that ICT solutions in the company may } \\
\text { not be used to their maximum capability }\end{array}$ & 0.768 & \multirow{3}{*}{0.759} \\
\hline & INA2 & $\begin{array}{l}\text { In the company, we are concerned that ICT may } \\
\text { become obsolete very rapidly }\end{array}$ & 0.586 & \\
\hline & INA3 & $\begin{array}{l}\text { In the company, we are concerned that ICT may } \\
\text { have to be personalized to work well }\end{array}$ & 0.666 & \\
\hline \multirow{3}{*}{$\begin{array}{l}\text { A lack of } \\
\text { reliability in } \\
\text { security (FCO) }\end{array}$} & FCO1 & $\begin{array}{l}\text { We are concerned that ICT may not be secure } \\
\text { and reliable to manage company information }\end{array}$ & 0.556 & \multirow{3}{*}{0.727} \\
\hline & FCO2 & $\begin{array}{l}\text { We are concerned that people who are not part } \\
\text { of the company could have access to digital } \\
\text { information without permission }\end{array}$ & 0.521 & \\
\hline & FCO3 & $\begin{array}{l}\text { We do not like to have company information } \\
\text { leaving the company's physical headquarters }\end{array}$ & 0.805 & \\
\hline \multirow{3}{*}{$\begin{array}{l}\text { ICT cost- } \\
\text { benefit } \\
\text { unbalance } \\
\text { (DES) }\end{array}$} & DES1 & $\begin{array}{l}\text { We worry that the benefits of ICT in the } \\
\text { company will not cover the investment made }\end{array}$ & 0.407 & \multirow{3}{*}{0.642} \\
\hline & DES2 & $\begin{array}{l}\text { Future costs to maintain and update ICT is one } \\
\text { of the company's concerns }\end{array}$ & 0.482 & \\
\hline & $\operatorname{DES} 3$ (**) $^{* *}$ & $\begin{array}{l}\text { Before implementing an ICT project in the company, we } \\
\text { evaluate the benefit-cost relation }\end{array}$ & 0.708 & \\
\hline
\end{tabular}

$\left(^{*}\right)$ Inverted scale

$(* *)$ Variable not included to obtain better reliability

Table 4. Analysis of scale reliability 
When conducting a principal component analysis, initially, a Kaiser, Meyer, Olkin Measure of Sampling Adequacy of 0.838 was identified. This indicates that the sample is adequate to conduct these types of factorial analysis. In Table 5, the factorial loads obtained for each item with rotated components are presented implementing varimax rotation. Following a priori criteria used for component extraction (Hair et al., 1999), 10 factors were defined for extraction. They correspond to the number of constructs proposed in the theoretical framework. The 10 factors extracted were able to explain 77.70 percent of the variance of the original items.

Since this study involves a sample size including more than 350 observations, a factorial load higher than 0.3 is considered significant to assure the convergent validity of each item and its corresponding construct (Hair et al., 1999). Likewise, it is expected that the factorial load be close to zero regarding other constructs to ensure its discriminant validity. In Table 5, you can see that factorial loads meet those criteria.

\begin{tabular}{|c|c|c|c|c|c|c|c|c|c|c|c|}
\hline \multirow{2}{*}{ Constructs } & \multirow{2}{*}{ Variable } & \multicolumn{10}{|c|}{ Factors } \\
\hline & & 1 & 2 & 3 & 4 & 5 & 6 & 7 & 8 & 9 & 10 \\
\hline \multirow{3}{*}{$\begin{array}{l}\text { Relative } \\
\text { advantage(REL) }\end{array}$} & $\alpha$ & 0.577 & 0.041 & -0.028 & 0.024 & -0.029 & -0.059 & 0.000 & 0.031 & 0.001 & 0.019 \\
\hline & REL2 & 0.556 & -0.014 & 0.016 & -0.041 & 0.018 & 0.051 & -0.034 & 0.034 & -0.023 & -0.026 \\
\hline & REL3 & 0.587 & -0.023 & 0.017 & 0.009 & 0.000 & 0.020 & 0.022 & -0.026 & 0.016 & 0.031 \\
\hline \multirow{3}{*}{ Observability (OBS) } & S1 & -0.033 & 0.543 & 0.077 & -0.097 & 0.015 & 0.143 & -0.037 & 0.108 & -0.069 & -0.107 \\
\hline & OBS2 & 0.028 & 0.599 & -0.045 & 0.047 & -0.036 & -0.043 & 0.001 & -0.040 & 0.095 & -0.045 \\
\hline & OBS3 & 0.000 & 0.574 & -0.032 & 0.028 & 0.009 & -0.058 & 0.043 & -0.081 & -0.084 & 0.142 \\
\hline \multirow{2}{*}{ Complexity (COJ) } & COJ1 & 0.010 & -0.008 & 0.709 & 0.001 & 0.032 & -0.004 & 0.025 & 0.028 & -0.011 & -0.035 \\
\hline & & -0.010 & -0.004 & 0.655 & 0.009 & -0.078 & 0.020 & 0.001 & -0.055 & -0.036 & 0.066 \\
\hline \multirow{3}{*}{$\begin{array}{l}\text { New business } \\
\text { opportunities(NOP) }\end{array}$} & & 0.003 & 0.028 & 0.036 & 0.606 & 0.024 & -0.039 & -0.024 & 0.026 & -0.074 & -0.008 \\
\hline & & -0.009 & -0.039 & -0.016 & 0.569 & -0.031 & 0.044 & 0.017 & -0.063 & -0.063 & 0.093 \\
\hline & & 0.004 & & -0.018 & 0.523 & 01 & 0.039 & 0.000 & & 0.110 & -0.133 \\
\hline \multirow{2}{*}{$\begin{array}{l}\text { Effective client } \\
\text { communication(CEF) }\end{array}$} & & -0.022 & -0.023 & 0.004 & 0.032 & 0.689 & -0.047 & -0.023 & 0.053 & -0.043 & -0.021 \\
\hline & & 0.015 & 0.013 & -0.038 & -0.035 & 0.672 & 0.075 & 0.037 & -0.051 & -0.013 & 0.061 \\
\hline \multirow{3}{*}{$\begin{array}{l}\text { Business Cost } \\
\text { Reduction (RCO) }\end{array}$} & & -0.007 & 0.000 & -0.097 & 0.070 & -0.047 & 0.490 & 0.070 & -0.076 & -0.015 & 0.146 \\
\hline & RCO2 & 0.004 & -0.017 & 0.018 & -0.002 & -0.004 & 0.593 & -0.031 & 0.024 & 0.099 & -0.031 \\
\hline & & 0.009 & 0.029 & 0.066 & -0.013 & 0.079 & 0.558 & -0.024 & 0.049 & 0.020 & -0.056 \\
\hline \multirow{2}{*}{$\begin{array}{l}\text { Government } \\
\text { incentives(IGO) }\end{array}$} & IGO1 & -0.009 & -0.007 & 0.026 & 0.018 & -0.018 & 0.001 & 0.682 & 0.039 & 0.003 & -0.024 \\
\hline & 2 & 0.004 & 0.013 & 0.002 & -0.022 & 0.026 & -0.008 & 0.708 & -0.002 & 0.022 & 0.016 \\
\hline \multirow{3}{*}{$\begin{array}{l}\text { Unsuitable ICT for the } \\
\text { business (INA) }\end{array}$} & & 0.053 & -0.018 & -0.011 & 0.019 & 0.048 & -0.014 & 0.059 & 0.670 & -0.058 & -0.135 \\
\hline & & -0.066 & -0.033 & 0.015 & -0.061 & -0.080 & 0.108 & -0.059 & 0.486 & -0.077 & 0.165 \\
\hline & A3 & -0.033 & 0.007 & -0.140 & 0.017 & -0.152 & 0.006 & 0.047 & 0.363 & -0.060 & 0.239 \\
\hline \multirow{3}{*}{$\begin{array}{l}\text { A lack of reliability in } \\
\text { security (FCO) }\end{array}$} & FCO1 & -0.031 & 0.073 & 0.118 & 0.050 & 0.063 & -0.083 & -0.090 & 0.201 & 0.306 & 0.188 \\
\hline & FCO2 & -0.005 & 0.059 & 0.054 & 0.045 & 0.105 & -0.153 & -0.036 & 0.283 & 0.429 & -0.022 \\
\hline & FCO3 & 0.001 & -0.026 & -0.042 & -0.036 & -0.056 & 0.071 & 0.028 & -0.089 & 0.786 & -0.019 \\
\hline \multirow{2}{*}{$\begin{array}{l}\text { ICT cost-benefit } \\
\text { unbalance (DES) }\end{array}$} & & 0.016 & -0.007 & 0.033 & 0.032 & 0.048 & -0.062 & 0.021 & -0.083 & 0.191 & 0.550 \\
\hline & DES2 & 0.012 & 0.000 & 0.004 & -0.028 & 0.016 & 0.032 & -0.017 & -0.007 & -0.116 & 0.679 \\
\hline
\end{tabular}

Rotated component matrix. Method: principal component with Varimax rotation

Table 5. Principal component analysis 


\section{Analysis and Results}

The results of the variance inflation factor analysis presented in Table 6 shows for all factors, that tolerance is higher than 0.1 and the values of the variance inflation factor are less than 10 (they vary from 1.29 to 1.92). This indicates that there are no problems of multicollinearity between independent variables to conduct a multiple regression analysis afterwards (Hair et al., 1999).

\begin{tabular}{|l|r|r|}
\hline Independent variables & Tolerance & VIF \\
\hline Relative advantage(REL) & 0.680783 & 1.47 \\
\hline Observability (OBS) & 0.778189 & 1.29 \\
\hline Complexity (COJ) & 0.601220 & 1.66 \\
\hline New business opportunities(NOP) & 0.602993 & 1.66 \\
\hline Effective client communication(CEF) & 0.641279 & 1.56 \\
\hline Business Cost Reduction (RCO) & 0.525241 & 1.90 \\
\hline Government incentives(IGO) & 0.691356 & 1.45 \\
\hline Unsuitable ICT for the business (INA) & 0.573429 & 1.74 \\
\hline A lack of reliability in security (FCO) & 0.542718 & 1.84 \\
\hline ICT cost-benefit unbalance (DES) & 0.521501 & 1.92 \\
\hline
\end{tabular}

Table 6. Result of the multicollinearity analysis of variables

The Table 7 highlights that the constructs that present a higher mean are relative advantage, benefits of new business opportunities, business cost reduction and effective client communication, and barriers associated to a lack of reliability in security and ICT not suitable for the business.

\begin{tabular}{|c|c|c|c|c|c|c|c|c|c|c|c|c|c|c|c|}
\hline \multicolumn{2}{|r|}{ Variable } & Type & Media & DE & 1 & 2 & 3 & 4 & 5 & 6 & 7 & 8 & 9 & 10 & 11 \\
\hline 1 & AdopICT & DV & 3.28 & 0.513 & 1.00 & & & & & & & & & & \\
\hline 2 & REL & IV & 4.70 & 0.523 & $0.23 *$ & 1.00 & & & & & & & & & \\
\hline 3 & OBS & IV & 3.52 & 0.967 & $0.09 *$ & $0.21 *$ & 1.00 & & & & & & & & \\
\hline 4 & $\mathrm{COJ}$ & IV & 2.05 & 0.978 & $-0.08 *$ & $-0.21 *$ & $0.28 *$ & 1.00 & & & & & & & \\
\hline 5 & NOP & IV & 4.15 & 0.773 & $0.43 *$ & $0.43^{*}$ & $0.21 *$ & $-0.15 *$ & 1.00 & & & & & & \\
\hline 6 & $\mathrm{CEF}$ & IV & 3.96 & 1.050 & $0.09 *$ & $0.21 *$ & $-0.10 *$ & $-0.49 *$ & $0.15 *$ & 1.00 & & & & & \\
\hline 7 & RCO & IV & 4.12 & 0.728 & $0.31 *$ & $0.51 *$ & $0.23 *$ & $-0.21 *$ & $0.61 *$ & $0.20 *$ & 1.00 & & & & \\
\hline 8 & IGO & IV & 1.82 & 0.975 & 0.02 & $-0.12 *$ & $0.20 *$ & $0.44 *$ & -0.00 & $-0.47 *$ & -0.03 & 1.00 & & & \\
\hline 9 & INA & IV & 3.45 & 0.903 & -0.07 & -0.04 & $0.11 *$ & $0.22 *$ & -0.07 & $-0.25 *$ & $-0.10 *$ & $0.14^{*}$ & 1.00 & & \\
\hline 10 & FCO & IV & 3.53 & 0.947 & $-0.15 *$ & -0.01 & $0.22 *$ & $0.31 *$ & $-0.08 *$ & $-0.23 *$ & $-0.09 *$ & $0.11 *$ & $0.56^{*}$ & 1.00 & \\
\hline 11 & DES & IV & 3.27 & 1.018 & -0.03 & -0.07 & $0.14 *$ & $0.28 *$ & $-0.08 *$ & $-0.25 *$ & $-0.17 *$ & $0.16^{*}$ & $0.60 *$ & $0.60 *$ & 1.00 \\
\hline
\end{tabular}

Notes. DV: Dependent variable; IV: Independent variable; SD: Standard deviation; $* \mathrm{p}<0.10$

Table 7. Statistical descriptions and correlations

The results of the multiple regression analysis between the independent variables that measure the three characteristics of the DOI model, benefits and barriers and the dependent variable, adoption of ICT are presented in Table 8 . This table shows regression with the variable "adoption of ICT" made up of 
the mean of 8 technologies (PCs, e-mail, LAN/WAN, Internet, Intranet, Web Page, Social Networks and e-commerce) and each one of these ICT individually.

In the compound model, there are three variables (one benefit and 2 barriers) which are significantly associated with the adoption of ICT by SMEs in Colombia. The benefit perceived by ICT is that they allow the creation of new business opportunities. Nevertheless, a lack of reliability on ICT security and the perception that an investment on ICT does not return the benefits expected for the business (costbenefit unbalance) become barriers negatively associated to the adoption of ICT. Upon finding these statistically significant relations in the compound model, we accepted hypotheses H4, H9 and H10.

While in the compound model, none of the three independent variables associated to the DOI model are significantly associated to the adoption of ICT in SMEs. Therefore, H1, H2 and H3 were rejected. Likewise, effective client communication, business cost reduction, government incentives and ICT unsuitable for the business did not predict significantly the adoption of ICT; therefore, H5, H6, H7 and H8 were rejected.

\begin{tabular}{|c|c|c|c|c|c|c|c|c|c|}
\hline Model & 1 & 2 & 3 & 4 & 5 & 6 & 7 & 8 & 9 \\
\hline V. Dep. & $\begin{array}{l}\text { Adop } \\
\text { ICT }\end{array}$ & PCs & Email & LAN & Internet & Intranet & $\begin{array}{l}\text { Web } \\
\text { Page }\end{array}$ & $\begin{array}{c}\text { Social } \\
\text { Networks }\end{array}$ & e-commerce \\
\hline REL & $\begin{array}{c}0.049 \\
(1.01)\end{array}$ & $\begin{array}{r}0.106^{* *} \\
(2.45)\end{array}$ & $\begin{array}{r}0.145^{* * *} \\
(3.38)\end{array}$ & $\begin{array}{c}0.214 \\
(1.60)\end{array}$ & $\begin{array}{r}0.091 * * \\
(2.24)\end{array}$ & $\begin{array}{l}-0.027 \\
(-0.18)\end{array}$ & $\begin{array}{l}0.036 \\
(0.38)\end{array}$ & $\begin{array}{c}0.096 \\
(0.79)\end{array}$ & $\begin{array}{r}-0.268^{*} \\
(-1.94)\end{array}$ \\
\hline OBS & $\begin{array}{l}0.004 \\
(0.15)\end{array}$ & $\begin{array}{l}-0.017 \\
(-0.80) \\
\end{array}$ & $\begin{array}{l}-0.021 \\
(-0.97)\end{array}$ & $\begin{array}{l}-0.087 \\
(-1.28)\end{array}$ & $\begin{array}{l}0.006 \\
(0.29)\end{array}$ & $\begin{array}{l}0.033 \\
(0.44)\end{array}$ & $\begin{array}{l}-0.006 \\
(-0.12) \\
\end{array}$ & $\begin{array}{l}0.095 \\
(1.54)\end{array}$ & $\begin{array}{l}0.028 \\
(0.40)\end{array}$ \\
\hline COJ & $\begin{array}{c}0.005 \\
(0.18)\end{array}$ & $\begin{array}{l}-0.000 \\
(-0.01)\end{array}$ & $\begin{array}{r}-0.065^{* * *} * \\
(-2.67)\end{array}$ & $\begin{array}{l}-0.024 \\
(-0.31)\end{array}$ & $\begin{array}{l}-0.013 \\
(-0.55)\end{array}$ & $\begin{array}{l}-0.002 \\
(-0.03)\end{array}$ & $\begin{array}{r}0.055 \\
(1.02)\end{array}$ & $\begin{array}{l}0.011 \\
(0.16)\end{array}$ & $\begin{array}{c}0.079 \\
(1.00)\end{array}$ \\
\hline NOP & $\begin{array}{r}0.237 * * * \\
(6.72)\end{array}$ & $\begin{array}{c}0.030 \\
(0.97)\end{array}$ & $\begin{array}{l}0.044 \\
(1.42)\end{array}$ & $\begin{array}{l}0.159 \\
(1.65)\end{array}$ & $\begin{array}{l}0.020 \\
(0.69)\end{array}$ & $\begin{array}{r}0.305^{* * *} \\
(2.89)\end{array}$ & $\begin{array}{r}0.467 * * * \\
(6.94)\end{array}$ & $\begin{array}{r}0.463^{* * *} \\
(5.31)\end{array}$ & $\begin{array}{r}0.404 * * * \\
(4.07)\end{array}$ \\
\hline CEF & $\begin{array}{c}0.008 \\
(0.33)\end{array}$ & $\begin{array}{l}0.017 \\
(0.78)\end{array}$ & $\begin{array}{l}0.007 \\
(0.31)\end{array}$ & $\begin{array}{l}0.037 \\
(0.54)\end{array}$ & $\begin{array}{c}0.026 \\
(1.26)\end{array}$ & $\begin{array}{l}-0.105 \\
(-1.39)\end{array}$ & $\begin{array}{r}0.063 \\
(1.31)\end{array}$ & $\begin{array}{l}-0.018 \\
(-0.29) \\
\end{array}$ & $\begin{array}{l}0.039 \\
(0.56)\end{array}$ \\
\hline RCO & $\begin{array}{c}0.048 \\
(1.20)\end{array}$ & $\begin{array}{l}0.007 \\
(0.20)\end{array}$ & $\begin{array}{l}0.020 \\
(0.58)\end{array}$ & $\begin{array}{c}0.158 \\
(1.43)\end{array}$ & $\begin{array}{l}-0.030 \\
(-0.91)\end{array}$ & $\begin{array}{r}0.205^{*} \\
(1.71)\end{array}$ & $\begin{array}{l}-0.065 \\
(-0.85)\end{array}$ & $\begin{array}{l}-0.085 \\
(-0.85)\end{array}$ & $\begin{array}{c}0.174 \\
(1.74)\end{array}$ \\
\hline IGO & $\begin{array}{c}0.019 \\
(0.72)\end{array}$ & $\begin{array}{r}0.008 \\
(0.33)\end{array}$ & $\begin{array}{l}0.010 \\
(0.42)\end{array}$ & $\begin{array}{c}0.048 \\
(0.67)\end{array}$ & $\begin{array}{l}-0.020 \\
(-0.92)\end{array}$ & $\begin{array}{l}0.075 \\
(0.96)\end{array}$ & $\begin{array}{l}-0.045 \\
(-0.89)\end{array}$ & $\begin{array}{l}-0.010 \\
(-0.16)\end{array}$ & $\begin{array}{c}0.084 \\
(1.14)\end{array}$ \\
\hline INA & $\begin{array}{l}-0.013 \\
(-0.43)\end{array}$ & $\begin{array}{r}0.029 \\
(1.05)\end{array}$ & $\begin{array}{l}-0.026 \\
(-0.96)\end{array}$ & $\begin{array}{l}-0.081 \\
(-0.95)\end{array}$ & $\begin{array}{l}0.004 \\
(0.17)\end{array}$ & $\begin{array}{l}-0.055 \\
(-0.60)\end{array}$ & $\begin{array}{l}0.046 \\
(0.78)\end{array}$ & $\begin{array}{l}-0.056 \\
(-0.73)\end{array}$ & $\begin{array}{l}0.031 \\
(0.36)\end{array}$ \\
\hline FCO & $\begin{array}{r}-0.102^{* * *} \\
(-3.35)\end{array}$ & $\begin{array}{l}0.002 \\
(0.08)\end{array}$ & $\begin{array}{l}-0.025 \\
(-0.94)\end{array}$ & $\begin{array}{l}-0.061 \\
(-0.74)\end{array}$ & $\begin{array}{l}-0.001 \\
(-0.04)\end{array}$ & $\begin{array}{r}-0.335^{* * *} \\
(-3.70)\end{array}$ & $\begin{array}{r}-0.139 * * \\
(-2.41)\end{array}$ & $\begin{array}{l}-0.081 \\
-1.08)\end{array}$ & $\begin{array}{r}-0.171 * * \\
(-2.00)\end{array}$ \\
\hline DES & $\begin{array}{r}0.070^{* *} \\
(2.44)\end{array}$ & $\begin{array}{l}-0.018 \\
(-0.70)\end{array}$ & $\begin{array}{r}0.090^{* * *} \\
(3.59) \\
\end{array}$ & $\begin{array}{r}0.150^{*} \\
(1.90)\end{array}$ & $\begin{array}{l}0.005 \\
(0.21)\end{array}$ & $\begin{array}{r}0.147^{*} \\
(1.71)\end{array}$ & $\begin{array}{c}0.078 \\
(1.42)\end{array}$ & $0.072(1.02)$ & $\begin{array}{l}0.035 \\
(0.44)\end{array}$ \\
\hline 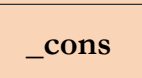 & $\begin{array}{r}1.956^{* * *} \\
(7.58)\end{array}$ & $\begin{array}{r}3.209^{* * *} \\
(14.02)\end{array}$ & $\begin{array}{r}3.003^{* * *} * \\
(13.27)\end{array}$ & $\begin{array}{c}1.110 \\
(1.57)\end{array}$ & $\begin{array}{r}3.473 * * * \\
(16.21)\end{array}$ & $\begin{array}{r}1.391^{*} \\
(1.80)\end{array}$ & $\begin{array}{r}1.395^{* * *} \\
(2.83)\end{array}$ & $\begin{array}{r}1.073^{*} \\
(1.68)\end{array}$ & $\begin{array}{c}0.997 \\
(1.37)\end{array}$ \\
\hline $\mathbf{N}$ & 474 & 474 & 474 & 474 & 474 & 474 & 474 & 474 & 474 \\
\hline R-sq & 0.212 & 0.036 & 0.112 & 0.057 & 0.035 & 0.100 & 0.151 & 0.103 & 0.090 \\
\hline adj. R-sq & 0.195 & 0.015 & 0.093 & 0.036 & 0.14 & 0.080 & 0.132 & 0.084 & 0.070 \\
\hline $\mathbf{F}$ & $12.49 * * *$ & $1.73 *$ & $5.83^{* * *}$ & $2.78^{* * *}$ & $1.68^{*}$ & $5.14 * * *$ & $8.22^{* * *}$ & $5.32 * * *$ & $4.56^{* * *}$ \\
\hline
\end{tabular}

Notes. t statistic in parenthesis; ${ }^{*} \mathrm{p}<0.1 ;{ }^{* *} \mathrm{p}<0.05 ;{ }^{* * *} \mathrm{p}<0.01$ 
Now, when we review statistically significant results of the adoption of ICT in the individual models of each one of the technologies shown in Table 8, you find that the adoption of technologies as PCs, company email and Internet, are positively related with the relative advantage of the DOI model, and ecommerce is negatively associated. Likewise, the adoption of a company e-mail is negatively associated to the factor of complexity of the DOI model.

On the other hand, the strong positive relation in the compound model between the adoptions of ICT a new business opportunities is highly influenced by the adoption of internet technologies, company web pages, social networks and ecommerce. Also, there is a big influence of Internet technologies, company webpages and e-commerce on the negative relation between the adoption of ICT in the compound model and the lack of reliability in ICT security and privacy. Finally, the technologies that most influence the relation found between the adoption of compound model ICT and the perception of ICT cost-benefit unbalance are e-mail, LANs and intranet.

Table 9 compares the results of the general multiple regression model between 10 independent variables and the dependent variable, adoption of ICT with four additional models considering classification variables as "size"and "sector", and another 4 models implementing the combinations of both variables. The variable, "size" takes a "micro" value for companies that have 1 to 10 employees and an SME value for companies that have more than 10 employees. The variable, "sector" takes the values of "industry" or "service".

When reviewing the results of the model for service companies in Table 9, we found that the independent variables of the model are statistically significant and associated to the adoption of ICT while for industry companies, just one variable was significant for the adoption of ICT. The common factor for both sectors corresponds to new business opportunities and lack of reliability on ICT, relative advantage, a return of the expected benefits on an ICT investment and decreased costs. 


\begin{tabular}{|c|c|c|c|c|c|c|c|c|c|}
\hline Model & 1 & 2 & 3 & 4 & 5 & 6 & 7 & 8 & 9 \\
\hline Sample & Total & Micro & SMEs & Service & Industry & $\begin{array}{c}\text { Micro } \\
\text { Service }\end{array}$ & $\begin{array}{c}\text { Micro } \\
\text { Industry }\end{array}$ & $\begin{array}{l}\text { SMEs } \\
\text { Service }\end{array}$ & $\begin{array}{l}\text { SMEs } \\
\text { Industry }\end{array}$ \\
\hline REL & $\begin{array}{c}0.049 \\
(1.01)\end{array}$ & $\begin{array}{l}0.096 \\
(1.59)\end{array}$ & $\begin{array}{l}-0.091 \\
(-1.11)\end{array}$ & $\begin{array}{r}0.116^{* *} \\
(2.05) \\
\end{array}$ & $\begin{array}{l}-0.111 \\
(-1.13)\end{array}$ & $\begin{array}{l}0.102 \\
(1.54)\end{array}$ & $\begin{array}{l}-0.002 \\
(-0.01)\end{array}$ & $\begin{array}{l}-0.011 \\
(-0.10) \\
\end{array}$ & $\begin{array}{l}-0.136 \\
(-1.00)\end{array}$ \\
\hline OBS & $\begin{array}{l}0.004 \\
(0.15)\end{array}$ & $\begin{array}{l}0.011 \\
(0.38)\end{array}$ & $\begin{array}{l}0.006 \\
(0.15)\end{array}$ & $\begin{array}{c}0.015 \\
(0.54)\end{array}$ & $\begin{array}{l}0.020 \\
(0.36)\end{array}$ & $\begin{array}{l}0.017 \\
(0.47)\end{array}$ & $\begin{array}{l}0.072 \\
(1.09)\end{array}$ & $\begin{array}{c}0.004 \\
(0.09)\end{array}$ & $\begin{array}{l}-0.049 \\
(-0.43)\end{array}$ \\
\hline COJ & $\begin{array}{l}0.005 \\
(0.18) \\
\end{array}$ & $\begin{array}{l}-0.022 \\
(-0.61) \\
\end{array}$ & $\begin{array}{c}0.036 \\
(0.84)\end{array}$ & $\begin{array}{l}0.020 \\
(0.63)\end{array}$ & $\begin{array}{l}-0.004 \\
(-0.07)\end{array}$ & $\begin{array}{l}-0.019 \\
(-0.46)\end{array}$ & $\begin{array}{l}-0.033 \\
(-0.41)\end{array}$ & $\begin{array}{l}0.078 \\
(1.62)\end{array}$ & $\begin{array}{l}0.036 \\
(0.39)\end{array}$ \\
\hline NOP & $\begin{array}{r}0.237 * * * \\
(6.72)\end{array}$ & $\begin{array}{r}0.226^{* * *} \\
(5.28) \\
\end{array}$ & $\begin{array}{r}0.220^{* * *} \\
(3.54) \\
\end{array}$ & $\begin{array}{r}0.231 * * * \\
(5.90)\end{array}$ & $\begin{array}{r}0.243^{* * *} * \\
(3.14) \\
\end{array}$ & $\begin{array}{r}0.235^{* * * *} \\
(4.73)\end{array}$ & $\begin{array}{r}0.199^{* *} \\
(2.29)\end{array}$ & $\begin{array}{r}0.198^{* * *} \\
(3.08)\end{array}$ & $\begin{array}{r}0.390^{* *} \\
(2.35)\end{array}$ \\
\hline CEF & $\begin{array}{l}0.008 \\
(0.33)\end{array}$ & $\begin{array}{c}0.019 \\
(0.59)\end{array}$ & $\begin{array}{l}-0.005 \\
(-0.14)\end{array}$ & $\begin{array}{l}0.032 \\
(1.13)\end{array}$ & $\begin{array}{l}-0.034 \\
(-0.65)\end{array}$ & $\begin{array}{l}0.043 \\
(1.15)\end{array}$ & $\begin{array}{l}-0.048 \\
(-0.67)\end{array}$ & $\begin{array}{l}0.017 \\
(0.40)\end{array}$ & $\begin{array}{l}-0.076 \\
(-0.75)\end{array}$ \\
\hline RCO & $\begin{array}{l}0.048 \\
(1.20) \\
\end{array}$ & $\begin{array}{l}0.049 \\
(1.02)\end{array}$ & $\begin{array}{l}0.004 \\
(0.05) \\
\end{array}$ & $\begin{array}{r}0.076^{*} \\
(1.66) \\
\end{array}$ & & $\begin{array}{l}0.076 \\
(1.34)\end{array}$ & $\begin{array}{l}-0.010 \\
(-0.10)\end{array}$ & $\begin{array}{c}0.062 \\
(0.78)\end{array}$ & $\begin{array}{l}-0.163 \\
(-0.98)\end{array}$ \\
\hline IGO & $\begin{array}{l}0.019 \\
(0.72) \\
\end{array}$ & $\begin{array}{l}0.029 \\
(0.89) \\
\end{array}$ & $\begin{array}{l}0.010 \\
(0.23) \\
\end{array}$ & $\begin{array}{l}0.014 \\
(0.48)\end{array}$ & $\begin{array}{l}0.013 \\
(0.23) \\
\end{array}$ & $\begin{array}{l}0.017 \\
(0.45)\end{array}$ & $\begin{array}{l}0.040 \\
(0.57)\end{array}$ & $\begin{array}{l}-0.011 \\
(-0.26) \\
\end{array}$ & $\begin{array}{l}-0.028 \\
(-0.19)\end{array}$ \\
\hline INA & $\begin{array}{l}-0.013 \\
(-0.43)\end{array}$ & $\begin{array}{l}-0.019 \\
(-0.51)\end{array}$ & $\begin{array}{l}0.012 \\
(0.22)\end{array}$ & $\begin{array}{l}0.009 \\
(0.26)\end{array}$ & $\begin{array}{l}-0.054 \\
(-0.84)\end{array}$ & $\begin{array}{l}-0.025 \\
(-0.57)\end{array}$ & $\begin{array}{l}-0.014 \\
(-0.19)\end{array}$ & $\begin{array}{r}0.094 \\
(1.61)\end{array}$ & $\begin{array}{l}-0.214 \\
(-1.62)\end{array}$ \\
\hline FCO & $\begin{array}{r}-0.102^{* * *} \\
(-3.35) \\
\end{array}$ & $\begin{array}{r}-0.085^{* *} \\
(-2.31) \\
\end{array}$ & $\begin{array}{r}-0.128^{* *} \\
(-2.40) \\
\end{array}$ & $\begin{array}{r}-0.130 * * * \\
(-3.81)\end{array}$ & $\begin{array}{l}-0.025 \\
(-0.39)\end{array}$ & $\begin{array}{r}-0.121 * * * \\
(-2.85)\end{array}$ & $\begin{array}{l}0.033 \\
(0.43)\end{array}$ & $\begin{array}{r}-0.121 * * \\
(-2.10) \\
\end{array}$ & $\begin{array}{l}-0.219 \\
(-1.66)\end{array}$ \\
\hline DES & $\begin{array}{r}0.070^{* *} \\
(2.44)\end{array}$ & $\begin{array}{r}0.069^{* *} \\
(2.04)\end{array}$ & $\begin{array}{c}0.052 \\
(0.92)\end{array}$ & $\begin{array}{r}0.078^{* *} \\
(2.46)\end{array}$ & $\begin{array}{l}0.040 \\
(0.64)\end{array}$ & $\begin{array}{r}0.102^{* * *} \\
(2.64)\end{array}$ & $\begin{array}{l}-0.021 \\
(-0.31)\end{array}$ & $\begin{array}{c}0.002 \\
(0.04)\end{array}$ & $\begin{array}{r}0.275^{*} \\
(1.76)\end{array}$ \\
\hline s & $\begin{array}{r}1.956^{* * *} \\
(7.58)\end{array}$ & $\begin{array}{r}1.653^{* * *} \\
(5.35)\end{array}$ & $\begin{array}{r}3.059 * * * \\
(6.43)\end{array}$ & $\begin{array}{r}1.412^{* * * *} \\
(4.71)\end{array}$ & $\begin{array}{r}3.013^{* * *} \\
(5.89)\end{array}$ & $\begin{array}{r}1.428^{* * *} \\
(3.95)\end{array}$ & $\begin{array}{r}2.307^{* * *} \\
(3.48)\end{array}$ & $\begin{array}{r}2.283^{* * *} \\
(3.94)\end{array}$ & $\begin{array}{r}4.062^{* * *} \\
(3.93)\end{array}$ \\
\hline $\mathbf{N}$ & 474 & 310 & 164 & 341 & 133 & 224 & 86 & 117 & 47 \\
\hline R-sq & 0.212 & 0.255 & 0.136 & 0.291 & 0.113 & 0.334 & 0.134 & 0.193 & 0.266 \\
\hline adj. R-sq & 0.195 & 0.230 & 0.080 & 0.270 & 0.040 & 0.303 & 0.019 & 0.117 & 0.062 \\
\hline $\mathbf{F}$ & $12.49 * * *$ & $10.23^{* * *}$ & $2.41 * *$ & $13.57 * * *$ & 1.55 & $10.69 * * *$ & 1.16 & $2.54 * * *$ & 1.31 \\
\hline
\end{tabular}

Notes. Dependent variable: AdopICT. t statistical in parenthesis; ${ }^{*} \mathrm{p}<0.1 ;{ }^{* *} \mathrm{p}<0.05 ;{ }^{* * *} \mathrm{p}<0.01$

Table 9. Multiple regression models per type of company

\section{Discussion and conclusions}

In view of the scarcity of empirical studies which analyze the adoption of technology in Colombian companies, this study provides a valuable contribution to the creation of knowledge when trying to explain the adoption of ICT by SMEs in Colombia comprehensively and quantitatively using a representative sample which allows a certain type of generalization of the results within the population we studied.

In this study, we have researched and analyzed the impact that 10 factors have on the adoption of ICT by SMEs in Colombia. To draw up the constructs, we began with Rogers' (1995) diffusion of innovation model selecting three of the five constructs he defined: Relative advantage, complexity and observability. They are complemented with other constructs that measure benefits and barriers perceived by companies when adopting ICT as new business opportunities, effective client communication, business cost reduction, government incentives, ICT unsuitable for the business, a lack 
of reliability in ICT security and ICT cost-benefit unbalance. The model designed for this resulting research was proven and validated statistically.

We formulated 10 hypotheses which were tested empirically using data taken from 474 Colombian micro companies and SMEs whose economic activities are industry and services.

The results of this research show that new business opportunities seem to be the most important benefit that affects Colombian SMEs' willingness to adopt ICT, and regarding the barriers that constrain the adoption of ICT, the most outstanding is a lack of reliability on ICT security, and ICT cost-benefit unbalance (when an investment on ICT does not yield the expected benefits).

When we analyze in depth comparing models per type of technology, additional factors arise like relative advantage, which affects positively Colombian SMEs' willingness to adopt e-mail, personal computers, intranet and e-commerce. Furthermore, complexity affects negatively the adoption of a company e-mail.

When comparing models per economic sector, we found that companies in the services sector where influenced by five factors when deciding to adopt ICT. They were relative advantage, new business opportunities, lack of reliability on ICT security, ICT cost-benefit unbalance (DES), and cost reduction. While companies in the industry sector were only influenced by the factor of new business opportunities.

The comparison of models by size of a company shows that there is a shared influence of two factors for the adoption of ICT. They are new business opportunities and a lack of reliability on ICT security. However, micro companies were also influenced by the fact that an investment on ICT does not yield the expected benefits.

This study has its own limitations because it is a cross-section research and this restricts the interpretations of causal relations between independent and dependent variables (just like you could do with a longitudinal study). Likewise, this study considers the fact that the generalization of the results is circumscribed to the geographical context and the type of companies interviewed, and we must bear in mind the fact that the grading of the scales was based on managers' perceptions instead of objective measures. Furthermore, due to the number of constructs introduced in the models and extension restrictions the questionnaire allowed, scales have been used, with less items than the originals and this may affect a bit the validity of the content. This limitation can be surpassed in future studies using the complete scales identified in the literature. 
While the results of this study do not seem to be strong confirming all the hypotheses, they may have important implications for the managers of SMEs, and for the different organizations included in different sectors, national or local government. This is an important associated benefit of the adoption of ICT besides starting to break the two barriers found in this research.

The fact, that new business opportunities are perceived as enablers of the adoption of ICT by SMEs in Colombia, has become a motivating element to embark on education and appropriation programs focused on ICT usefulness for the business.

While it is true that a lack of trust on ICT safety is a generalized perception of small, medium and large companies, it must be combated principally in SMEs because the fear of the vulnerability of the privacy of their information or the availability of their information inhibits the adoption of these technologies.

Likewise, plans and forms must be created to show the bounties, benefits and the returns of ICT investments by SMEs because it is quite clear that the way software and telecommunications suppliers and the government itself have tried to sell ICT potentials in SMEs; it is not the best because it underlies between the idea that the investment on ICT does not yield enough benefits for their businesses.

Finally, this research proposes future research lines covering the impact that the adoption of ICT has on the profitability of companies, longitudinal studies (verifying in the future how evolve the adoption of ICT in SMEs, and its preceding factors), adoption of new ICT (LTE, big data, social media, IoT, etc.), or the use of other models for the adoption of technology like TAM, TRA or TPB instead of DOI to analyze the adoption of ICT in SMEs in Colombia.

\section{References}

Agarwal, R., \& Prasad, J. (1997). The role of innovation characteristics and perceived voluntariness in the acceptance of information technologies. Decision sciences, 28(3), 557-582. http://dx.doi.org/10.1111/j.1540-5915.1997.tb01322.x

Ajzen, I. (1985). From intentions to actions: A theory of planned behavior. In J. Kuhl \& J. Beckman (Eds.), Action-control: From cognition to behavior (pp. 11-39). Berlin Heidelberg: Springer. http://dx.doi.org/10.1007/978-3-642-69746-3_2 
Ajzen, I., \& Fishbein, M. (1980). Understanding attitudes and predicting social behaviour. Englewood Cliffs, NJ: Prentice-Hall.

Al-Qirim, N. (2006). Personas of E-Commerce Adoption in Small Businesses in New Zealand. Journal of Electronic Commerce in Organizations, 4(3), 18-45. http://dx.doi.org/10.4018/jeco.2006070102

Aljifri, H.A., Pons, A., \& Collins, D. (2003). Global e-commerce: a framework for understanding and overcoming the trust barrier. Information Management \& Computer Security, 11(3), 130-138. http://dx.doi.org/10.1108/09685220310480417

Alkalbani, S., Rezgui, Y., Vorakulpipat, C., \& Wilson, I.E. (2013). ICT adoption and diffusion in the construction industry of a developing economy: The case of the sultanate of Oman. Architectural Engineering and Design Management, 9(1), 62-75. http://dx.doi.org/10.1080/17452007.2012.718861

Alshamaila, Y., Papagiannidis, S., \& Li, F. (2013). Cloud computing adoption by SMEs in the north east of England: A multi-perspective framework. Journal of Enterprise Information Management, 26(3), 250-275. http://dx.doi.org/10.1108/17410391311325225

APEC - Asia Pacific Economic Cooperation (1999). SME Electronic Commerce Study. Singapore: APEC Publishing.

Arendt, L. (2008). Barriers to ICT adoption in SMEs: how to bridge the digital divide?. Journal of Systems and Information Technology, 10(2), 93-108. http://dx.doi.org/10.1108/13287260810897738

Bayo-Moriones, A., \& Lera-López, F. (2007). A firm-level analysis of determinants of ICT adoption in Spain. Technovation, 27(6), 352-366. http://dx.doi.org/10.1016/j.technovation.2007.01.003

Beatty, R.C., Shim, J., \& Jones, M.C. (2001). Factors influencing corporate web site adoption: A timebased assessment. Information \& management, 38(6), 337-354. http://dx.doi.org/10.1016/S03787206(00)00064-1

Benham, H.C., \& Raymond, B.C. (1996). Information technology adoption: Evidence from a voice mail introduction. ACM SIGCPR Computer Personnel, 17(1), 3-25. http://dx.doi.org/10.1145/227005.227006

Blackwood, F. (1997). E-commerce benefits for small firms. San Francisco Business Times, 12(12), 25.

Brancheau, J.C., \& Wetherbe, J.C. (1990). The adoption of spreadsheet software: Testing innovation diffusion theory in the context of end-user computing. Information Systems Research, 1(2), 115-143. http://dx.doi.org/10.1287/isre.1.2.115

Cabarcas Álvarez, A., Martelo Gómez, R.J., \& Puello Marrugo, P. (2013). TICs en las Pymes: Herramienta útil para aumentar la competitividad. Ingeniator, 3(5), 10-17. 
Cámara Colombiana de Informática y Tecnología \& Fedesarollo (2013). El papel de las TIC en el desarrollo de la pequeña empresa: reflexiones de política a la luz del caso colombiano. Retrieved from: Fedesarrollo. Centro de investigación económica y social website: http://www.fedesarrollo.org.co/wpcontent/uploads/2013/12/TIC diciembre 2013.pdf

Consoli, D. (2012). Literature analysis on determinant factors and the impact of ICT in SMEs. ProcediaSocial and Behavioral Sciences, 62, 93-97. http://dx.doi.org/10.1016/j.sbspro.2012.09.016

Cragg, P.B. (1990). Information technology and small firm performance. Loughborough University.

Cron, W.L., \& Sobol, M.G. (1983). The relationship between computerization and performance: A strategy for maximizing the economic benefits of computerization. Information \& management, 6(3), 171-181. http://dx.doi.org/10.1016/0378-7206(83)90034-4

Chapman, P., James-Moore, M., Szczygiel, M., \& Thompson, D. (2000). Building internet capabilities in SMEs. Logistics Information Management, 13(6), 353-361. http://dx.doi.org/10.1108/09576050010355662

Chaudhury, A., \& Bharati, P. (2008). IT Outsourcing Adoption by Small and Medium Enterprises: A Diffusion of Innovation Approach. Paper presented at the AMCIS 2008 Proceedings. Paper 390. Available online at: http://aisel.aisnet.org/amcis2008/390

Chesher, M., \& Skok, W. (2000). Roadmap for successful information technology transfer for small businesses. Paper presented at the Proceedings of the 2000 ACM SIGCPR conference on Computer personnel research. http://dx.doi.org/10.1145/333334.333338

Davis, F.D. (1989). Perceived usefulness, perceived ease of use, and user acceptance of information technology. MIS quarterly, 13(3), 319-340. http://dx.doi.org/10.2307/249008

ECTEL (2009). Use of ICT by SMEs in the ECTEL member states. Retrieved from ECTEL website: http://www.ectel.int/index.php/resources/publications

Fillis, I., Johannson, U., \& Wagner, B. (2004). Factors impacting on e-business adoption and development in the smaller firm. International Journal of Entrepreneurial Behavior \& Research, 10(3), 178-191. http://dx.doi.org/10.1108/13552550410536762

Ghobakhloo, M., Arias-Aranda, D., \& Benitez-Amado, J. (2011). Adoption of e-commerce applications in SMEs. Industrial Management \& Data Systems, 111(8), 1238-1269. http://dx.doi.org/10.1108/02635571111170785

Gibbs, J.L., \& Kraemer, K.L. (2004). A cross-country investigation of the determinants of scope of ecommerce use: An institutional approach. Electronic Markets, 14(2), 124-137. http://dx.doi.org/10.1080/10196780410001675077 
Grandon, E.E., \& Mykytyn Jr, P.P. (2004). Theory-based instrumentation to measure the intention to use electronic commerce in small and medium sized businesses. The Journal of Computer Information Systems, 44(3), 44-57. http://dx.doi.org/10.1016/j.im.2003.12.010

Grandon, E.E., \& Pearson, J.M. (2004). Electronic commerce adoption: an empirical study of small and medium US businesses. Information \& management, 42(1), 197-216.

Hair, J.F., Anderson, R.E., Tatham, R.L., \& Black, W.C. (1999). Análisis multivariante. Madrid: Prentice Hall.

Harindranath, G., Dyerson, R., \& Barnes, D. (2008). ICT in Small Firms: Factors Affecting the Adoption and Use of ICT in Southeast England SMEs. Paper presented at the ECIS 2008 Proceedings. Paper 167.

He, Q., Duan, Y., Fu, Z., \& Li, D. (2006). An innovation adoption study of online e-payment in Chinese companies. Journal of Electronic Commerce in Organizations, 4(1), 48-69. http://dx.doi.org/10.4018/jeco.2006010104

Hempel, P.S., \& Kwong, Y.K. (2001). B2B e-Commerce in emerging economies: i-metal. com's nonferrous metals exchange in China. Journal of Strategic Information Systems, 10(4), 335-355. http://dx.doi.org/10.1016/S0963-8687(01)00058-0

Hepworth, M., \& Ryan, J. (2000). Small firms in Europe's developing information society. In K. Ducatel, J. Webster \& W. Herrmann (Eds.), The Information Society in Europe. Work and Life in an Age of Globalization (pp. 73-96). Lanham, Md.: Rowman \& Littlefield Publishers.

Hsieh, C.-T., Jones, C., \& Lin, B. (2008). The new business potential with mobile commerce. International Journal of Mobile Communications, 6(4), 436-455. http://dx.doi.org/10.1504/IJMC.2008.018052

Hussin, H., \& Noor, R.M. (2005). Innovating business through e-commerce: Exploring the willingness of Malaysian SMEs. Paper presented at the Proceedings of the Second International Conference on Innovation in IT.

Iacovou, C.L., Benbasat, I., \& Dexter, A.S. (1995). Electronic data interchange and small organizations: Adoption and impact of technology. MIS quarterly, 19(4), 465-485. http://dx.doi.org/10.2307/249629

ITU (2015). ICT Facts and Figures. Retrieved from: International Telecommunication Union website: http://www.itu.int/en/ITU-D/Statistics/Documents/facts/ICTFactsFigures2015.pdf

Ives, B., \& Olson, M.H. (1984). User involvement and MIS success: A review of research. Management science, 30(5), 586-603. http://dx.doi.org/10.1287/mnsc.30.5.586 
Kendall, J.D., Tung, L.L., Chua, K.H., Ng, C.H.D., \& Tan, S.M. (2001). Receptivity of Singapore's SMEs to electronic commerce adoption. Journal of Strategic Information Systems, 10(3), 223-242. http://dx.doi.org/10.1016/S0963-8687(01)00048-8

Kumbakara, N. (2008). Managed IT services: The role of IT standards. Information Management \& Computer Security, 16(4), 336-359. http://dx.doi.org/10.1108/09685220810908778

Lee, J. (2004). Discriminant analysis of technology adoption behavior: A case of Internet technologies in small businesses. Journal of Computer Information Systems, 44(4), 57.

Lettieri, E., \& Masella, C. (2005). Measuring the value and sustainability of internet-based ICT in healthcare organisations. International journal of healthcare technology and management, 7(3-4), 319-332.

Limthongchai, P., \& Speece, M. (2003). The effect of perceived characteristics of innovation on ecommerce adoption by SMEs in Thailand. Paper presented at the Proceedings of the Seventh International Conference on Global Business and Economic Development, Bangkok, Thailand. Available online at: http://ssrn.com/abstract $=2565392$

Lin, H.-F., \& Lin, S.-M. (2008). Determinants of e-business diffusion: A test of the technology diffusion perspective. Technovation, 28(3), 135-145. http://dx.doi.org/10.1016/j.technovation.2007.10.003

Lucchetti, R., \& Sterlacchini, A. (2004). The adoption of ICT among SMEs: Evidence from an Italian survey. Small Business Economics, 23(2), 151-168. http://dx.doi.org/10.1023/B:SBEJ.0000027667.55821.53

Marston, S., Li, Z., Bandyopadhyay, S., Zhang, J., \& Ghalsasi, A. (2011). Cloud computing-The business perspective. Decision Support Systems, 51(1), 176-189. http://dx.doi.org/10.1016/j.dss.2010.12.006

Miller, M. (2008). Cloud computing: Web-based applications that change the way you work and collaborate online. Inidanapolis: QUE publishing.

Ministerio de TIC (2014). Logros Plan Vive Digital. Available online at: http://micrositios.mintic.gov.co/vivedigital/logros-plan/index.php

Ministerio de TIC (2015). Boletín Trimestral de las TIC Cifras Cuarto Trimestre de 2014. Retrieved from: Colombia TIC Vive Digital website: http://colombiatic.mintic.gov.co/602/articles-8598 archivo pdf.pdf

Mndzebele, N. (2013). The Effects of Relative Advantage, Compatibility and Complexity in the Adoption of EC in the Hotel Industry. International Journal of Computer and Communication Engineering, 2(4), 473-476. http://dx.doi.org/10.7763/IJCCE.2013.V2.229

Moghaddam, B.K., \& Khatoon-Abadi, A. (2013). Factors affecting ICT adoption among rural users: A case study of ICT Center in Iran. Telecommunications Policy, 37(11), 1083-1094. http://dx.doi.org/10.1016/j.telpol.2013.02.005 
Molla, A., \& Licker, P.S. (2005). Perceived e-readiness factors in e-commerce adoption: An empirical investigation in a developing country. International Journal of Electronic Commerce, 10(1), 83-110.

Moore, G.C., \& Benbasat, I. (1991). Development of an instrument to measure the perceptions of adopting an information technology innovation. Information Systems Research, 2(3), 192-222. http://dx.doi.org/10.1287/isre.2.3.192

Mykytyn Jr, P.P., \& Harrison, D.A. (1993). The application of the theory of reasoned action to senior management and strategic information systems. Information Resources Management Journal, 6(2), 15. http://dx.doi.org/10.4018/irmj.1993040102

Narayanasamy, K., Santhapparaj, A., \& Eze, U.C. (2008). An empirical study of website adoption among Small and Medium Enterprises (SMEs) in Malaysia. Communications of the IBIMA, 2(28), $339-351$.

Ntemana, T.J., \& Olatokun, W. (2012). Analyzing the Influence of Diffusion of Innovation Attributes on Lecturers' Attitude Towards Information and Communication Technologies. Human Technology, 8(2), 179-197. http://dx.doi.org/10.17011/ht/urn.201211203034

Nunnally, J.C., \& Bernstein, I.H. (1994). Psychometric Theory. New York: McGraw-Hill.

OECD (2002). Information Technology Outlook 2002. Paris: OECD. http://dx.doi.org/10.1787/it_outlook-2002en

Orduz, R. (2011). Internet en Colombia: Los cambios de una década (2000-2010). Retrieved from: Colombia Digital website: http://www.colombiadigital.net/opinion/columnistas/los-numeros-de-las-tic/item/643internet-en-colombia-los-cambios-de-una-d\%C3\%A9cada-2000-2010.html

Parisot, A.H. (1995). Technology and teaching: The adoption and diffusion of technological innovations by a community college faculty. Montana State University, Bozeman, MT.

Premkumar, G. (2003). A meta-analysis of research on information technology implementation in small business. Journal of organizational computing and electronic commerce, 13(2), 91-121. http://dx.doi.org/10.1207/S15327744JOCE1302_2

Premkumar, G., \& King, W.R. (1994). Organizational characteristics and information systems planning: an empirical study. Information Systems Research, 5(2), 75-109. http://dx.doi.org/10.1287/isre.5.2.75

Premkumar, G., Ramamurthy, K., \& Nilakanta, S. (1994). Implementation of electronic data interchange: An innovation diffusion perspective. Journal of Management Information Systems, 11(2), 157-186. http://dx.doi.org/10.1080/07421222.1994.11518044 
Ramdani, B., \& Kawalek, P. (2007). SMEs \& IS innovations adoption: a review \& assessment of previous research. Academia. Revista Latinoamericana de Administración, (39), 47-70.

Riemenschneider, C.K., \& Mckinney, V.R. (2001). Assessing belief differences in small business adopters and non-adopters of web-based e-commerce. Journal of Computer Information Systems, 42(2), 101.

Rogers, E.M. (1995). Diffusion of innovations (4 ed.). New York, NY: Free Press.

Rouibah, K., Khalil, O.E., \& Hassanien, A.E. (2009). Emerging Markets and E-Commerce in Developing Economies. Hershey, New York: Information Science Reference. http://dx.doi.org/10.4018/978-1-60566$100-1$

Saffu, K., Walker, J.H., \& Hinson, R. (2007). An empirical study of perceived strategic value and adoption constructs: The Ghanaian case. Management Decision, 45(7), 1083-1101. http://dx.doi.org/10.1108/00251740710773925

Sahin, I. (2006). Detailed Review of Rogers' Diffusion of Innovations Theory and Educational Technology-Related Studies Based on Rogers' Theory. Turkish Online Journal of Educational Technology, $5(2), 14-23$.

Scupola, A. (2001). Adoption of Internet-based electronic commerce in Southern Italian SMEs. Paper presented at the 1 st Nordic Workshop on Electronic Commerce, Halmstad, Sweden.

Slegers, C., Singh, S., \& Hall, J. (1998). Small business and electronic commerce: an Australian survey. Melbourne: CIRCIT at RMIT.

Sokolov, I.P. (2009). Cloud Computing: Overview, Concepts and Business Deployment Scenarios. Vienna

Spanos, Y.E., Prastacos, G.P., \& Poulymenakou, A. (2002). The relationship between information and communication technologies adoption and management. Information \& management, 39(8), 659-675. http://dx.doi.org/10.1016/S0378-7206(01)00141-0

Syed, S.A., Khatibi, A., Ismail, H., \& Ahmad, S. (2005). Perceived benefits of e-commerce adoption in the electronic manufacturing companies in Malaysia. Journal of Social Sciences, 1(3), 188-193. http://dx.doi.org/10.3844/jssp.2005.188.193

Tan, K.S., Chong, S.C., Lin, B., \& Eze, U.C. (2009). Internet-based ICT adoption: Evidence from Malaysian SMEs. Industrial Management \& Data Systems, 109(2), 224-244. http://dx.doi.org/10.1108/02635570910930118 
Tan, K.S., \& Eze, U.C. (2008). An empirical study of internet-based ICT adoption among Malaysian SMEs. Communications of the IBIMA, 1(1), 1-12.

Tan, M., \& Teo, T.S. (2000). Factors influencing the adoption of Internet banking. Journal of the Association for Information Systems, 1(1), 1-42.

Teo, T.S.H., \& Pok, S.H. (2003). Adoption of WAP-enabled mobile phones among Internet users. Omega, 31(6), 483-498. http://dx.doi.org/10.1016/j.omega.2003.08.005

Thong, J.Y., \& Yap, C.-S. (1995). CEO characteristics, organizational characteristics and information technology adoption in small businesses. Omega, 23(4), 429-442. http://dx.doi.org/10.1016/03050483(95)00017-I

Thong, J.Y., Yap, C.-S., \& Raman, K. (1994). Engagement of external expertise in information systems implementation. Journal of Management Information Systems, 11(2), 209-231. http://dx.doi.org/10.1080/07421222.1994.11518046

Thong, J.Y.L. (1999). An integrated model of information systems adoption in small businesses. Journal of Management Information Systems, 15(4), 187-214. http://dx.doi.org/10.1080/07421222.1999.11518227

Tiwana, A., \& Bush, A.A. (2007). A comparison of transaction cost, agency, and knowledge-based predictors of IT outsourcing decisions: A US-Japan cross-cultural field study. Journal of Management Information Systems, 24(1), 259-300. http://dx.doi.org/10.2753/MIS0742-1222240108

Tornatzky, L.G., Fleischer, M., \& Chakrabarti, A.K. (1990). The processes of technological innovation. Lexington: Lexington Books.

Van Slyke, C., Belanger, F., \& Comunale, C.L. (2004). Factors influencing the adoption of web-based shopping: the impact of trust. ACM Sigmis Database, 35(2), 32-49. http://dx.doi.org/10.1145/1007965.1007969

Venkatesh, V., Morris, M.G., Davis, G.B., \& Davis, F.D. (2003). User acceptance of information technology: Toward a unified view. MIS quarterly, 27(3), 425-478.

Wang, Y., Meister, D., \& Wang, Y. (2008). Relative Advantage and Perceived Usefulness: The Adoption of Competing ICT. Paper presented at the DIGIT 2008 Proceedings. Paper 6. Available online at: http://aisel.aisnet.org/digit2008/6

Weiss, J.A., \& Dale, B.C. (1998). Diffusing against mature technology: Issues and strategy. Industrial Marketing Management, 27(4), 293-304. http://dx.doi.org/10.1016/S0019-8501(97)00062-X 
Wymer, S.A., \& Regan, E.A. (2005). Factors influencing e-commerce adoption and use by small and medium businesses. Electronic Markets, 15(4), 438-453. http://dx.doi.org/10.1080/10196780500303151

Yap, C.-S., Thong, J.Y.L., \& Raman, K.S. (1994). Effect of government incentives on computerisation in small business. European Journal of Information Systems, 3(3), 191-206.

http://dx.doi.org/10.1057/ejis.1994.20

Yap, C.S., \& Walsham, G. (1986). A survey of information technology in the UK service sector. Information \& management, 10(5), 267-274. http://dx.doi.org/10.1016/0378-7206(86)90029-7

Zhang, P., Aikman, S.N., \& Sun, H. (2008). Two types of attitudes in ICT acceptance and use. International Journal of Human-Computer Interaction, 24(7), 628-648.

http://dx.doi.org/10.1080/10447310802335482

Zhu, K., \& Kraemer, K.L. (2005). Post-adoption variations in usage and value of e-business by organizations: Cross-country evidence from the retail industry. Information Systems Research, 16(1), 61-84. http://dx.doi.org/10.1287/isre.1050.0045

\section{Versión en español}

Título: Análisis de los factores que influyen en la adopción de las TIC por parte de las PYMES de Colombia

\section{Resumen}

Objeto: El objetivo de este artículo es analizar los factores que influyen en la adopción de las Tecnologías de Información y Comunicaciones (TIC) por parte de las Pymes en Colombia.

Diseño/metodología/enfoque: Para comprobar empíricamente las hipótesis aplicamos un cuestionario a una muestra de 474 pymes de Colombia sobre la adopción de TIC y algunas variables independientes identificadas en la literatura, luego realizamos una serie de modelos de regresión múltiple con los datos obtenidos. 
Aportaciones y resultados: Desarrollamos un modelo que identifica una relación positiva entre las nuevas oportunidades de negocio que brindan las TIC y el nivel de adopción de estas. Igualmente, se identifica que la falta de confianza en la seguridad y privacidad de las TIC y la percepción de desbalance entre los costos y beneficios de las TIC, tienen un impacto negativo sobre la adopción de estas tecnologías.

Limitaciones: Este estudio presenta las limitaciones propias de una investigación de corte transversal, lo cual restringe la posibilidad de hacer análisis dinámicos entre variables independientes y dependientes. De igual forma, la generalización de los resultados se circunscribe al contexto geográfico y tipo de empresas encuestadas.

Implicaciones prácticas: Los resultados proporcionan valiosas contribuciones a la toma de decisiones por parte de los directivos de las PYME y las políticas públicas que fomenten la adopción de las TIC en las PYME, a partir del análisis de los beneficios y barreras encontrados en este estudio.

Originalidad / Valor añadido: Ante la escasez de trabajos empíricos que analicen la adopción tecnológica en empresas de países emergentes, este estudio brinda un valioso aporte de generación de conocimiento al intentar explicar la adopción de TIC con una muestra representativa de Pymes en Colombia, identificando de forma cuantitativa el impacto de beneficios y barreras de estas tecnologías.

Palabras clave: Adopción tecnológica, Difusión de innovación, TIC, Pymes, Colombia

Códigos JEL: O32, O33, M15

\section{Introducción}

Las tecnologías de información y de comunicación (TIC), en particular el uso de Internet y los servicios Web, están cambiando rápidamente la forma tradicional de hacer negocios entre las empresas. Con la progresiva globalización de la economía, las TIC son una de las herramientas más versátiles para que las pequeñas y medianas empresas puedan competir de forma rentable en mercados más grandes y dinámicos. 
El interés continuo y creciente en la adopción de las TIC también se atribuye al crecimiento exponencial en el número de usuarios de Internet en todo el mundo, con un mayor incremento reportado de los usuarios en los países en desarrollo, especialmente cuando se compara Latinoamérica con EE.UU., Europa y Asia. De hecho, en la actualidad, 3,200 millones de personas están usando internet, de las cuales 2000 millones provienen de países en desarrollo (ITU, 2015).

Si bien los países con mayor PIB per cápita, tasa de alfabetización, infraestructura de telecomunicaciones bien establecida y estabilidad política, disfrutan de una mayor difusión de internet, la brecha con los países en desarrollo se ha empezado a disminuir, en primer lugar por las tasas de penetración de Internet en los primeros y de crecimiento en los segundos, y en segundo lugar, porque nuevas tecnologías de banda ancha móvil empiezan a ser más asequibles para países en vía de desarrollo (ITU, 2015).

Por ejemplo, en países como Colombia, las conexiones de internet de banda ancha vienen creciendo a una tasa del 20.4\% interanual y la penetración de Internet en a diciembre de 2014 fue del 22.3\% (10.6 millones de suscriptores) (Ministerio de TIC, 2015), lo cual equivale aproximadamente a la quinta parte de la población, en comparación con los 4.4 millones de suscriptores en 2010 o los 874 mil suscriptores que había en el año 2000 (Orduz, 2011).

El gobierno colombiano ha venido desarrollando el Plan Vive Digital 2010 - 2014 y su extensión (Plan Vive Digital 2.0, 2014 - 2018) muy enfocado en la masificación del acceso a internet. Por ejemplo, con el proyecto Red Nacional de Fibra Óptica se multiplicó por cinco el número de municipios conectados a través de redes de fibra óptica y la subasta de espectro radioeléctrico permitió tener cobertura de $4 \mathrm{G}$ en el 50\% del país (Ministerio de TIC, 2014). Por lo tanto, se percibe una seria atención en el desarrollo de la infraestructura de telecomunicaciones y el entorno de las TIC, con el fin de garantizar que el país pueda avanzar rápidamente en la era de la información.

Quizá la tecnología de información que más ha penetrado a las pequeñas y medianas empresas (Pymes) es Internet. De hecho, en 2010 solo el 7\% de microempresas (Mipymes) estaban conectadas a Internet y a 2014 el 60.6\% ya utilizaban Internet para comprar suministros, transacciones financieras, mercadeo, etc. (Ministerio de TIC, 2014).

En Colombia, el 99.9\% de las empresas son Mipymes, lo que corresponde a cerca 1,6 millones de unidades empresariales, y tal y como ocurre en otras latitudes, estas empresas concentran el $81 \%$ de los empleados, mientras que su contribución al PIB es de apenas 37\% (Cámara Colombiana de Informática y Tecnología \& Fedesarollo, 2013). Aunque queda clara la creciente penetración del uso de Internet en 
las Pymes colombianas, es necesario que éstas alineen las TIC con sus objetivos de negocio e incluyan herramientas tecnológicas como la Web 2.0, como alternativas esenciales dentro de su actividad o de su plan de productividad (Cabarcas Álvarez, Martelo Gómez \& Puello Marrugo, 2013). Es importante que las Pymes contemplen el uso y adopción de las TIC para llegar a un mayor grupo de clientes potenciales a nivel nacional y global.

Mientras que varios autores han identificado diversos factores que afectan la adopción o no adopción de sitios web y de comercio electrónico, sigue habiendo lagunas que deben abordarse. Los estudios realizados hasta el momento proporcionan un enfoque estrecho de las Pymes, principalmente hacia la adopción del comercio electrónico.

Son múltiples los modelos teóricos que se han utilizado en trabajos previos para explicar el comportamiento de los usuarios en la adopción de nuevas innovaciones tecnológicas, tales como el modelo de difusión de la innovación (DOI - Difussion of Innovations) que ha ganado popularidad en este campo (Rogers, 1995; Tan, Chong, Lin \& Eze, 2009), o la teoría de la acción razonada, la teoría del comportamiento planificado y el modelo de aceptación de la tecnología, que en algunos estudios se combinan para la construcción de nuevos modelos (Benham \& Raymond, 1996; Limthongchai \& Speece, 2003; Tan \& Teo, 2000), lo cual ha dado lugar a diferentes resultados.

Aunque los trabajos previos han compartido cierta proximidad geográfica en cuanto a los países analizados, se ha identificado que varios estudios que utilizan el modelo DOI han mostrado resultados contradictorios (Hussin \& Noor, 2005; Kendall, Tung, Chua, Ng \& Tan, 2001; Limthongchai \& Speece, 2003). Además, hay escasez de investigación combinando las variables del modelo DOI con otros constructos asociados a los beneficios y barreras de la adopción de las TIC.

Desarrollar un estudio de este tipo en un país en desarrollo como Colombia es un factor de alta relevancia. Si bien el gobierno ha estado invirtiendo activamente en el desarrollo de la infraestructura necesaria que ha contribuido al creciente número de usuarios de Internet y la promoción de la prestación de servicios basados en Internet, también es cierto que las limitaciones tecnológicas que enfrentan las Pymes son debidas en parte, a la falta de educación y habilidades en TIC, que es un medio importante para obtener ventaja competitiva en este mercado global (Cámara Colombiana de Informática y Tecnología \& Fedesarollo, 2013).

Por tanto, esta investigación se llevó a cabo para responder a las cuestiones antes mencionadas. Este estudio tiene como objetivo analizar los factores que influyen en el grado de adopción de las TIC por parte de las Pymes en Colombia. 
En el contexto de este trabajo, se entiende por adopción de TIC la definición propuesta por Tan et al. (2009) sobre las TIC basadas en Internet, entendiéndose como el uso de tecnologías, tales como hardware, software y red por parte de las Pymes para conectarse y usar Internet. Aunque las TIC basadas en Internet se consideran nueva innovación, la terminología no es la que ha sido utilizada por otros autores al realizar investigaciones relacionadas con la tecnología (Chapman, James-Moore, Szczygiel \& Thompson, 2000; Hsieh, Jones \& Lin, 2008; Kumbakara, 2008; Lettieri \& Masella, 2005; Lucchetti \& Sterlacchini, 2004). En este trabajo, por tanto, las terminologías de adopción de TIC y adopción de TIC basadas en Internet, se utilizan indistintamente.

\section{Marco teórico}

Las TIC han sido abordadas desde diferentes ámbitos. Las Naciones Unidas las han considerado desde el enfoque del desarrollo socioeconómico, la Unión Internacional de Telecomunicaciones (ITU) las ha definido como un sector económico y los Estados Unidos y otros países han relacionado fuertemente el uso de las TIC al ámbito de la educación. Sin embargo, desde el punto de vista de los negocios, las TIC son tecnologías usadas por personas y organizaciones para el procesamiento de su información y propósitos de comunicación (Zhang, Aikman \& Sun, 2008).

La adopción de las TIC basadas en Internet hacen alusión a cómo una empresa aprovecha Internet para innovar en los negocios y al uso de sitios Web como una plataforma de comunicaciones al interior de la empresa y entre las partes interesadas de la misma (Narayanasamy, Santhapparaj \& Eze, 2008; Tan et al., 2009)

Los adoptantes tardíos de las TIC se caracterizan por un menor grado de penetración de las mismas, lo que significa que la difusión de las TIC en las Pymes puede seguir un patrón del modelo de difusión de tecnología de curva S de Rogers (Lucchetti \& Sterlacchini, 2004). Hepworth \& Ryan (2000) argumentan que las Pymes siguen el patrón de normalización de difusión de las TIC, por lo que el desarrollo de éstas da crédito a la idea de que Internet es una plataforma de crecimiento para las Pymes.

Respecto a otros estudios que se han hecho sobre la adopción de TIC en las Pymes, Bayo-Moriones y Lera-López (2007) centran su atención en las características que identifican a los determinantes claves de la adopción de las TIC en las empresas españolas. Prestaron especial atención a variables tales como la edad de los trabajadores, la presión competitiva, los mercados internacionales, las estrategias competitivas basadas en bajo costo o diferenciación y la certificación ISO, entre otras. 
Por otro lado, al revisar la literatura de los diferentes estudios de adopción de TIC en Pymes que se han hecho en otros países distintos a Colombia, se encuentra que se han utilizado diversos marcos conceptuales y teóricos de adopción de tecnología. Sin embargo, según Rouibah, Khalil y Hassanien (2009), básicamente son cuatro las teorías que se han utilizado ampliamente para explicar la adopción tecnológica:

- TAM - Modelo de Aceptación Tecnológica (Davis, 1989);

- TRA - Teoría de la Acción Razonada (Ajzen \& Fishbein, 1980);

- $\quad$ TPB - Teoría del Comportamiento Planeado (Ajzen, 1985);

- DOI - Difusión de Innovaciones (Rogers, 1995). Estas teorías se describen brevemente en la Tabla 1.

Existen otros modelos de adopción tecnológica menos conocidos como TOE - Marco de trabajo Tecnología Organización Ambiente (Tornatzky, Fleischer \& Chakrabarti, 1990), PERM - Modelo de Alistamiento Electrónico Percibido (Molla \& Licker, 2005) y el modelo UTAUT - Teoría Unificada de Aceptación y Uso de Tecnología (Venkatesh, Morris, Davis \& Davis, 2003), que pretende unificar varios modelos reconocidos de aceptación, difusión y adopción de tecnología. Sin embargo, estos han sido utilizados en menor proporción para explicar la adopción de TIC en las organizaciones (ver Tabla 1).

\begin{tabular}{|l|l|l|}
\hline \multicolumn{1}{|c|}{ Autores (Año) } & \multicolumn{1}{|c|}{ Modelo } & \multicolumn{1}{c|}{ Descripción del modelo } \\
\hline Davis (1989) & $\begin{array}{l}\text { TAM - Modelo de } \\
\text { Aceptación Tecnológica } \\
\text { (Technology Acceptance Mode) }\end{array}$ & $\begin{array}{l}\text { Cuando a un usuario se le presenta una nueva tecnología, la } \\
\text { 'utilidad percibida' y 'facilidad de uso percibida' influyen } \\
\text { notablemente sobre cómo y cuándo los usuarios la utilizarán. }\end{array}$ \\
\hline Ajzen y Fishbein (1980) & $\begin{array}{l}\text { TRA - Teoría de la Acción } \\
\text { Razonada (Theory of Reasoned } \\
\text { Action) }\end{array}$ & $\begin{array}{l}\text { Esta teoría busca predecir el comportamiento y la actitud de } \\
\text { los individuos. Los componentes de TRA son 'intención } \\
\text { comportamental', 'actitud' y 'norma subjetiva'. }\end{array}$ \\
\hline Ajzen (1985) & $\begin{array}{l}\text { TPB - Teoría del } \\
\text { comportamiento Planeado } \\
\text { (Theory of Planned Bebavior) }\end{array}$ & $\begin{array}{l}\text { Teoría psicológica que vincula creencias y comportamientos } \\
\text { en los individuos. A los tres componentes de la Teoría de } \\
\text { Acción Razonada se agrega el constructo 'Control } \\
\text { Comportamental Percibido'. Los cuatro constructos } \\
\text { determinan las intenciones comportamentales del individuo. }\end{array}$ \\
\hline Rogers (1995) & $\begin{array}{l}\text { DOI - Modelo de Difusión } \\
\text { de Innovación (Difussion of } \\
\text { Innovation) }\end{array}$ & $\begin{array}{l}\text { La teoría de difusión de innovaciones indica que los } \\
\text { adoptantes tempranos evalúan una innovación desde su } \\
\text { ventaja relativa, compatibilidad, complejidad, verificabilidad y } \\
\text { observabilidad. }\end{array}$ \\
\hline Tornatzky et al. (1990) & $\begin{array}{l}\text { TOE - Marco de trabajo } \\
\text { Tecnología Organización } \\
\text { Ambiente (Technology } \\
\text { Organization Environment } \\
\text { Framework) }\end{array}$ & $\begin{array}{l}\text { Una empresa adopta e implementa innovaciones tecnológicas } \\
\text { influenciada por variables del contexto tecnológico } \\
\text { (tecnologías internas y externas), del contexto organizacional } \\
\text { (características y recursos de la firma) y del contexto ambiental } \\
\text { (tamaño y estructura de la industria). }\end{array}$ \\
\hline
\end{tabular}




\begin{tabular}{|l|l|l|}
\hline \multicolumn{1}{|c|}{ Autores (Año) } & \multicolumn{1}{|c|}{ Modelo } & \multicolumn{1}{c|}{ Descripción del modelo } \\
\hline Molla y Licker (2005) & $\begin{array}{l}\text { PERM - Modelo de } \\
\text { Alistamiento electrónico } \\
\text { Percibido (Perceived eReadiness } \\
\text { Moded }\end{array}$ & $\begin{array}{l}\text { La adopción del comercio electrónico en las organizaciones } \\
\text { depende de dos dimensiones: POER (Perceived organizational } \\
\text { eReadiness) y PEER (Perceived external eReadiness). Los } \\
\text { constructos de POER son: conciencia, recursos, compromiso. } \\
\text { Los constructos de PEER son: fuerzas del mercado, gobierno, } \\
\text { industrias de apoyo. }\end{array}$ \\
\hline Venkatesh et al. (2003) & $\begin{array}{l}\text { UTAUT - Teoría Unificada } \\
\text { de Aceptación y Uso de } \\
\text { Tecnología (Unified Theory of } \\
\text { Acceptance and Use of } \\
\text { Technology) }\end{array}$ & $\begin{array}{l}\text { Modelo de aceptación tecnológica que busca explicar las } \\
\text { intenciones del usuario para utilizar un sistema de información } \\
\text { y su subsecuente comportamiento a partir de tres } \\
\text { determinantes de la intención de uso (expectativa de } \\
\text { desempeño, expectativa de esfuerzo, influencia social) y un } \\
\text { determinante de comportamiento de uso (condiciones } \\
\text { facilitadoras). }\end{array}$ \\
\hline $\begin{array}{l}\text { Bayo-Moriones y Lera- } \\
\text { López (2007); Spanos, } \\
\begin{array}{l}\text { Prastacos y Poulymenakou } \\
\text { (2002) }\end{array}\end{array}$ & $\begin{array}{l}\text { Adopción de TIC a nivel de } \\
\text { la empresa }\end{array}$ & $\begin{array}{l}\text { La adopción de TIC en empresas depende del ambiente, el } \\
\text { capital humano, la organización interna, la estrategia y las } \\
\text { características de la firma. }\end{array}$ \\
\hline
\end{tabular}

Tabla 1. Modelos de adopción tecnológica

Para el presente estudio de adopción de TIC en las Pymes de Colombia no se utilizó el modelo TAM porque no refleja completamente las influencias sociales en un contexto organizacional (Alkalbani, Rezgui, Vorakulpipat \& Wilson, 2013), sino que busca explicar la intención de un individuo por desarrollar el comportamiento en cuestión (Grandon \& Mykytyn Jr, 2004). Tampoco se usó el modelo TRA porque está más orientado a predecir el logro de objetivos (Riemenschneider \& McKinney, 2001). Igualmente, no se utiliza el modelo TPB porque está más enfocado en explicar y predecir las intenciones comportamentales de los gerentes y no tanto la adopción de tecnología a nivel organizacional (Mykytyn Jr \& Harrison, 1993).

De los anteriores modelos de adopción tecnológica, el modelo DOI (Rogers, 1995), es uno de los más utilizados para explicar la adopción tecnológica en las organizaciones, entendiéndose como un tipo de difusión de innovación al interior de las compañías (Brancheau \& Wetherbe, 1990; Hussin \& Noor, 2005; Kendall et al., 2001; Syed, Khatibi, Ismail \& Ahmad, 2005; Teo \& Pok, 2003; Van Slyke, Belanger \& Comunale, 2004), incluso algunos trabajos han combinado los constructos del modelo DOI con los de otros modelos como el TRA, TPB y TAM (Benham \& Raymond, 1996; Limthongchai \& Speece, 2003; Tan \& Teo, 2000). La Tabla 2 ilustra los resultados de algunos de los estudios de adopción de TIC en Pymes que se han basado en el modelo DOI. 


\begin{tabular}{|l|l|}
\hline \multicolumn{1}{|c|}{ Autores (Año) } & \multicolumn{1}{c|}{ Resultados } \\
\hline Kendall et al. (2001) & $\begin{array}{l}\text { Se investigó el e-commerce como nueva innovación entre las Pymes de Singapur. Los resultados } \\
\text { indican que la ventaja relativa, compatibilidad y verificabilidad son factores que afectan la adopción } \\
\text { de e-commerce. La observabilidad y la complejidad no son significativas. }\end{array}$ \\
\hline $\begin{array}{l}\text { Beatty, Shim y Jones } \\
(2001)\end{array}$ & $\begin{array}{l}\text { Se analizaron los factores que influyen en la adopción de sitios Web corporativos en 286 Pymes de } \\
\text { Estados Unidos. Las variables independientes fueron: beneficios percibidos, complejidad, } \\
\text { compatibilidad y soporte gerencial. Las de mayor significancia fueron beneficios percibidos (ventaja } \\
\text { relativa) y complejidad. }\end{array}$ \\
\hline $\begin{array}{l}\text { Limthongchai y } \\
\text { Speece (2003) }\end{array}$ & $\begin{array}{l}\text { Se estudió la adopción del e-commerce entre dueños, presidentes y CEOs de Pymes en Tailandia. } \\
\text { Además de las cinco características de DOI, agregaron la seguridad o confidencialidad. La ventaja } \\
\text { relativa, compatibilidad, seguridad o confidencialidad y observabilidad están positivamente } \\
\text { correlacionadas con la adopción del e-commerce, mientras que la complejidad tuvo correlación } \\
\text { negativa. }\end{array}$ \\
\hline $\begin{array}{l}\text { Hussin y Noor } \\
\text { (2005) }\end{array}$ & $\begin{array}{l}\text { Se exploró la disposición de las Pymes de Malasia al adoptar e-commerce entre los CEOs. La } \\
\text { ventaja relativa, la observabilidad y la complejidad fueron significativamente relacionadas con la } \\
\text { adopción de e-commerce, mientras que verificabilidad y compatibilidad no fueron significativas. }\end{array}$ \\
\hline Tan et al. (2009) & $\begin{array}{l}\text { Se investigó la adopción de las TIC basadas en Internet en 406 Pymes de Malasia. La ventaja } \\
\text { relativa, compatibilidad, observabilidad y seguridad estuvieron significativamente relacionadas de } \\
\text { forma positiva con la adopción de TIC en las Pymes, mientras que la complejidad tuvo una } \\
\text { asociación negativa. }\end{array}$ \\
\hline $\begin{array}{l}\text { Moghaddam y } \\
\text { Khatoon-Abadi } \\
\text { (2013) }\end{array}$ & $\begin{array}{l}\text { Se investigaron los factores que afectan la adopción de TIC de los usuarios rurales en el centro de } \\
\text { Irán. Se comprobó que las características de innovación de DOI tuvieron un efecto positivo y } \\
\text { significativo sobre la adopción de TIC por parte de dichos usuarios, con excepción de la variable } \\
\text { compatibilidad. }\end{array}$ \\
\hline
\end{tabular}

Tabla 2. Estudios previos de adopción de TIC en Pymes basados en el modelo DOI

En DOI, la difusión se entiende como el proceso social por medio del cual se comunica una innovación con el tiempo a través de ciertos canales entre los miembros de un sistema social (Rogers, 1995). Innovación, por otro lado, se define como una idea, práctica u objeto que se percibe como nuevo por una unidad individual o de otro tipo de adopción (Tan et al., 2009).

De acuerdo con este modelo, son cinco las influencias claves en el comportamiento de aceptación de una innovación por parte del usuario: ventaja relativa, complejidad, compatibilidad, observabilidad y verificabilidad (He, Duan, Fu \& Li, 2006).

Rogers (1995) describió de la siguiente forma cada una de estas cinco influencias clave o características importantes percibidas de la innovación:

- Ventaja relativa - el grado en que se percibe que la innovación es mejor que lo que se reemplaza.

- Compatibilidad - el grado en que la innovación es coherente con los valores existentes, experiencias pasadas y necesidades.

- Complejidad - el grado en que la innovación es difícil de entender y utilizar. 
- Verificabilidad - el grado en que la innovación se puede someter a experimentación o ensayo.

- Observabilidad - el grado de visibilidad de los nuevos resultados de la innovación.

Si bien muchos investigadores han usado el modelo DOI con sus cinco características de innovación, también es cierto que muchos estudios han probado que las características de compatibilidad y verificabilidad no suelen ser significativas al explicar la adopción de TIC en las Pymes (Hussin \& Noor, 2005; Moghaddam \& Khatoon-Abadi, 2013; Ntemana \& Olatokun, 2012; Tan et al., 2009).Por las razones anteriores, para el presente estudio de adopción de TIC en las Pymes de Colombia, se usaron sólo tres de las cinco características de innovación de DOI (ventaja relativa, observabilidad y complejidad), como marco de referencia para la adopción de TIC en las Pymes y se complementó el modelo con algunos constructos de beneficios y barreras de adopción de TIC.

\subsection{Ventaja Relativa}

La ventaja relativa es entendida como "el grado en el cual una innovación es percibida mejor que su predecesora" (Moore \& Benbasat, 1991). Es decir, que según la teoría de difusión de innovaciones, al adoptar una nueva innovación (en este caso una nueva TIC), los adoptantes potenciales (en este caso las Pymes), evalúan la ventaja relativa de ésta en términos de las eficiencias percibidas de dicha innovación respecto de las tecnologías o procedimientos actuales (Rogers, 1995).

La ventaja relativa es vista como una condición importante que influye sobre la decisión de adopción desde el contexto tecnológico y también es considerada como un indicador central para la adopción de nuevos sistemas de información (Alshamaila, Papagiannidis \& Li, 2013). El impacto de la ventaja relativa sobre la adopción de tecnología ha sido ampliamente investigado en estudios previos (Gibbs \& Kraemer, 2004; Iacovou, Benbasat \& Dexter, 1995; Lee, 2004; Premkumar \& King, 1994; Ramdani \& Kawalek, 2007; Thong, 1999). Se ha demostrado que cuando los negocios perciben una ventaja relativa en una innovación, entonces la probabilidad de dicha adopción se incrementará (Lee, 2004; Thong, Yap \& Raman, 1994; Thong, 1999).

La ventaja relativa es una función de la utilidad percibida de tecnologías nuevas y existentes, lo cual permite incorporar a la organización la influencia de otras tecnologías que de otra manera serían ignoradas (Wang, Meister \& Wang, 2008). Una decisión de adopción racional en una organización requiere que se evalúen los posibles beneficios de la nueva tecnología para el negocio. 
Las TIC representan una variedad de ganancias para las compañías que las adopten. Por ejemplo, de acuerdo con Miller (2008), la computación en la nube (un tipo de TIC), puede ofrecer muchas ventajas relacionadas con capacidad, confiabilidad y flexibilidad. Dicha tecnología baja considerablemente los costos de entrada de las Pymes para acceder a una gran cantidad de recursos computacionales en relativamente cortos periodos de tiempo, lo que les permite a los pequeños negocios concentrarse en el mercado sin la necesidad de inversiones de capital en hardware (Marston, Li, Bandyopadhyay, Zhang \& Ghalsasi, 2011). En este mismo sentido, Lin y Lin (2008) sostienen que la tecnología web es más probable de ser adoptada cuando las organizaciones perciben que ésta le ayudará con el intercambio de información de negocio. Así, desde una perspectiva de capacidades de TIC, las ventajas relativas de éste tipo de tecnologías para los negocios son casi dadas por hecho (Sokolov, 2009).

En términos generales entonces, la adopción de TIC debe estar positivamente influenciada por la ventaja relativa, por lo tanto se propone la siguiente hipótesis:

H1. Existe una relación positiva entre la adopción de TIC en las Pymes y la ventaja relativa de dichas tecnologías.

\subsection{Observabilidad}

La observabilidad se define como el grado en el cual los resultados de una innovación son visibles para otros. La mayoría de las innovaciones estudiadas en la investigación de difusión de innovaciones fueron temas tecnológicos (Rogers, 1995). La observabilidad es difícil de establecer en un mercado que ya esté dominado por una tecnología establecida. Por lo tanto será importante establecer observabilidad en un nicho de mercado o en una nueva aplicación de la tecnología antes de intentar retar a la tecnología dominante en su mercado y aplicación nativa (Weiss \& Dale, 1998).

En la actualidad, la observación de tecnologías TIC como por ejemplo el comercio electrónico, es relativamente fácil tanto para los consumidores como para las industrias adoptantes. El comercio electrónico incrementa la visibilidad de la compañía porque el sitio Web puede ser visto como un lugar para comprar. Los sitios Web permiten que los negocios estén abiertos 7x24 para millones de clientes y proveedores potenciales en Internet (Blackwood, 1997).

Con tecnologías como Internet, los clientes y proveedores pueden visitar el sitio Web de la Pyme para buscar información general con una rápida respuesta mediante correo electrónico u otro medio, en 
cualquier momento y desde cualquier lugar. Esto crea conveniencia y flexibilidad para que las Pymes establezcan relaciones con vendedores y compradores.

Si las Pymes pueden tener visibilidad de los beneficios de la adopción de TIC, es muy probable que adopten dichas tecnologías. De acuerdo con Slegers, Singh y Hall (1998), una de las barreras más importantes para acceder a servicios de Internet es la falta de conciencia. Es to nos lleva a formular la siguiente hipótesis:

H2. Existe una relación positiva entre la adopción de TIC en las Pymes y la visibilidad percibida del uso de dichas tecnologías.

\subsection{Complejidad}

Rogers (1995) define la complejidad como "el grado en el cual una innovación es percibida como difícil de usar". Adoptar nuevas tecnologías puede enfrentar a las Pymes a retos en términos de cambio en los procesos en los cuales interactúa para desarrollar su negocio. Las nuevas tecnologías deben ser amigables y fáciles de usar con el fin de incrementar la tasa de adopción (Parisot, 1995; Sahin, 2006).

Algunos autores sugieren que las nuevas ideas que son fáciles de entender serán adoptadas más rápidamente que las innovaciones que requieren que el adoptante desarrolle nuevos conocimientos, habilidades y entendimiento (Premkumar, Ramamurthy \& Nilakanta, 1994). Así mismo, los sistemas que son percibidos como más fáciles de usar y menos complejos, tienen mayor posibilidad de ser aceptados y utilizados por usuarios potenciales (Agarwal \& Prasad, 1997).

En muchos estudios se ha encontrado que la complejidad es un factor significativo en la decisión de adopción (Chaudhury \& Bharati, 2008; Harindranath, Dyerson \& Barnes, 2008; Tiwana \& Bush, 2007). En contraste, con las demás características de innovación de DOI, éste factor está negativamente asociado a la probabilidad de adopción (mientras más compleja la tecnología, menor será la probabilidad de adopción).

De acuerdo a la revisión de literatura sobre la complejidad, podemos formular la siguiente hipótesis:

H3. Existe una relación negativa entre la adopción de TIC en las Pymes y la complejidad de implementar o usar dichas tecnologías. 


\subsection{Nuevas Oportunidades de Negocios (Beneficios)}

Las organizaciones deben reconocer que la adopción de la innovación, o bien ofrece soluciones a los problemas existentes o bien presenta nuevas oportunidades de producción, tales como el aumento de la productividad y la mejora de la eficiencia operativa (Zhu \& Kraemer, 2005). Las organizaciones adoptan una tecnología cuando ven la necesidad de dicha tecnología, creyendo que aprovechará una oportunidad de negocio o cerrará una brecha en el desempeño organizacional (Mndzebele, 2013).

Así mismo, existen oportunidades y beneficios potenciales para el negocio cuando las Pymes implementan tecnologías como el comercio electrónico. Entre las oportunidades que obtienen está el fortalecimiento de la relación con los clientes, alcanzar nuevos mercados, optimizar procesos de negocio, reducir costos, mejorar el conocimiento del negocio, atraer inversionistas y crear nuevos productos y servicios. También, esta tecnología representa una oportunidad para que las Pymes compensen sus debilidades tradicionales en áreas como el acceso a nuevos mercados y recolectar y difundir información a escala internacional, lo cual mejora la comunicación y crea mayor flexibilidad de empleo (Scupola, 2001).

La tecnología avanza a una velocidad tan rápida que, sin lugar a dudas ofrece vastas oportunidades a las Pymes para acceder a mercados internacionales y para participar en la cadena de suministro global, siempre y cuando adopten TICs (Fillis, Johannson \& Wagner, 2004). Para poder hacer esto, los dueños o gerentes de las Pymes deben equiparse a sí mismos con suficiente conocimiento en este campo.

De acuerdo con lo anterior, sin lugar a dudas, la globalización y el rápido cambio tecnológico, incluyendo el uso de Internet y el comercio electrónico, pueden traer nuevas oportunidades de negocios para las Pymes (Scupola, 2001). En este sentido, Tan y Eze (2008) encontraron en un estudio que la adopción de TIC entre las Pymes provee nuevas oportunidades de negocio y acceso a información y conocimiento del mercado. Por lo tanto, podemos establecer la siguiente hipótesis:

H4. Existe una relación positiva entre la adopción de TIC en las Pymes y su potencial para generar nuevas oportunidades de negocios. 


\subsection{Comunicación Efectiva con los Clientes (Beneficios)}

Narayanasamy et al. (2008) menciona que una organización ha adoptado TICs cuando usan Internet mediante sitios Web como plataforma de comunicaciones entre y dentro de organizaciones y sus partes interesadas. Más aún, si un sitio Web contiene una plataforma de comercio electrónico, su interfaz puede virtualmente habilitar cualquier tipo de pedido de un cliente.

Algunos autores indican que la adopción de TIC puede contribuir a una estrategia de calidad en las organizaciones. Por ejemplo, Spanos et al. (2002) indican que las TIC les permiten a las empresas alcanzar una ventaja diferenciadora al asegurar las relaciones con los clientes a través de una mejor calidad y mayor habilidad para responder rápidamente a los cambios del mercado.

En este sentido, también Tan et al. (2009) encontraron que la adopción de TIC entre las Pymes se convierte en una efectiva herramienta de comunicación con los clientes. Por lo tanto, podemos establecer la siguiente hipótesis:

H5. Existe una relación positiva entre la adopción de TIC en las Pymes y el aumento de la efectividad de la comunicación con los clientes.

\subsection{Reducción de los Costos del Negocio (Beneficios)}

Hay una creencia de que tecnologías como el comercio electrónico contribuyen al avance de los negocios en los países en desarrollo, el cual es jalonado por el potencial de internet y las tecnologías de comunicaciones al reducir los costos de las transacciones, haciendo a un lado los intermediarios y facilitando los vínculos hacia cadenas de suministro globales (Hempel \& Kwong, 2001; Molla \& Licker, 2005).

También, de acuerdo con Tan et al. (2009), la adopción de las TIC basadas en Internet ofrece beneficios para las Pymes en términos de reducción de costos, por ejemplo costos de correspondencia. Así mismo, Mndzebele (2013) encontró en su estudio que los hoteles que adoptaban el comercio electrónico tenían dentro de sus beneficios percibidos la reducción de costos.

Los anteriores argumentos permiten formular la siguiente hipótesis:

H6. Existe una relación positiva entre la adopción de TIC en las Pymes y la reducción de costos para las Pymes. 


\subsection{Incentivos Gubernamentales (Beneficios)}

Basado en la revisión de literatura de varios autores, Consoli (2012) menciona que las políticas públicas constituyen un factor determinante para la adopción de las TIC en las Pymes. De hecho, los gobiernos juegan un rol significativo en promover la adopción de TIC en los países. La buena infraestructura de red, una sana competencia entre proveedores de redes, facilidad de préstamos, esquemas y programas de aplicación, son algunas de las iniciativas que los gobiernos pueden adoptar para alentar el uso de las TIC (Tan \& Eze, 2008).

Por eso, recientemente ha habido un incremento dramático en el número de compañías de soluciones de negocios en Tailandia, como consecuencia de la promoción del comercio electrónico por parte del gobierno de ese país (Limthongchai \& Speece, 2003).

Yap, Thong y Raman (1994) aseguran que si bien la participación en un programa de computarización gubernamental no resulta en sistemas de información más efectivos, los incentivos gubernamentales sí alientan a las Pymes que les falta recursos financieros y técnicos, para que sistematicen sus operaciones. La provisión de subsidios, préstamos de bajo interés, seminarios y experiencia técnica pueden compensar la falta de recursos y experiencia interna de las Pymes. Muchos negocios pequeños no se hubieran sistematizado y no hubiesen sido exitosos, de no ser por dichos incentivos.

El impacto positivo de las TIC en la productividad ha sido ampliamente demostrado, y una de las principales líneas de acción del gobierno en la búsqueda de la mejora de la competitividad de sus economías, es la promoción de éstas tecnologías entre las organizaciones (Bayo-Moriones \& LeraLópez, 2007).

Los anteriores argumentos nos llevan a formular la siguiente hipótesis:

H7. Existe una relación positiva entre la adopción de TIC en las Pymes y los incentivos gubernamentales para su adopción. 


\subsection{TIC inadecuadas para el negocio (Barreras)}

Entre las barreras identificadas para la adopción de TIC en las Pymes, está la falta de idoneidad de las TIC para los negocios (Tan et al., 2009; Tan \& Eze, 2008).

En un estudio llevado a cabo por ECTEL (2009), se identifica que los negocios pueden no ver la relevancia del uso de Internet para las transacciones si ellos creen que sus productos y servicios no son adecuados para el comercio en línea. La falta de información y conocimiento de las soluciones TIC disponibles también pueden contribuir a esta limitante percibida. De hecho, la OECD (2002) ha mencionado que la principal razón dada por los negocios para no comprometerse en el comercio electrónico es que éste no es adecuado para la naturaleza de sus negocios.

En Colombia a su vez, según la Cámara Colombiana de Informática y Tecnología \& Fedesarollo (2013), el 83.6\% de las microempresas en el país han reconocido que la principal razón para no usar Internet es que no se necesita para sus negocios.

Lo anterior nos lleva a plantear la siguiente hipótesis:

H8. Existe una relación negativa entre la adopción de TIC en las Pymes y la poca adecuación de las TICs para el negocio.

\subsection{Falta de confianza en la seguridad de las TIC (Barreras)}

Muchos estudios han identificado a la seguridad o confidencialidad de las TIC como un factor significativo que influye en la adopción de las mismas (Tan et al., 2009). Por ejemplo, Aljifri, Pons y Collins (2003), identificaron varios aspectos que impactan el nivel de confianza en el comercio electrónico y cómo esto afecta la posibilidad de hacer negocios en línea entre empresas, por lo cual consideraron que la seguridad de la información era una de las principales barreras que afectan el ecommerce en los países en desarrollo.

También, mediante evaluaciones sobre la percepción de las Pymes respecto a los inhibidores de la adopción de comercio electrónico por éstas, se ha encontrado que uno de los factores más influyentes era la preocupación sobre seguridad (APEC - Asia Pacific Economic Cooperation, 1999)

Así mismo, aunque las barreras para la aplicación de las TIC en las Pymes varían según el sector económico y el país, existen unas barreras comunes a todas las compañías, dentro de las cuales se 
encuentran los factores de seguridad y confianza (seguridad y confiabilidad de los sistemas de comercio electrónico, la incertidumbre de los métodos de pago en línea y los marcos legales) (Arendt, 2008).

Por su parte, en una encuesta, aquellas Pymes que respondieron que no adoptarían tecnologías de ecommerce, indicaron como una de las principales razones para esto la seguridad de la información (Wymer \& Regan, 2005).

Existen además otros estudios sobre la adopción de TIC en los cuales utilizaron la seguridad y privacidad como una dimensión importante a evaluar, complementando el modelo DOI, entre ellos se destacan los trabajos de Tan y Teo (2000), Limthongchai y Speece (2003) y Hussin y Noor (2005).

En términos generales entonces, la adopción de las TIC en las Pymes parece estar influenciada negativamente por los factores de seguridad, por lo tanto se propone la siguiente hipótesis:

H9. Existe una relación negativa entre la adopción de TIC en las Pymes y la falta de confianza en la seguridady privacidad de las mismas.

\subsection{Alta inversión en TIC sin retorno de beneficios (Barreras)}

Es difícil tener un análisis costo beneficio del impacto de los sistemas computacionales en las organizaciones. Aunque Ives y Olson (1984) intentaron definir el éxito de los sistemas de información como el beneficio organizacional agregado procedente de éstos en comparación con inversiones alternativas, la verdad es que varios estudios han fracasado en encontrar evidencia de que el uso de computadores o tecnologías relacionadas mejoren el desempeño de las Pymes (Cragg, 1990; Cron \& Sobol, 1983; Yap \& Walsham, 1986).

En muchas Pymes las TIC no son percibidas como generadoras de valor y los negocios medianos y pequeños creen que los costos de TIC no cubren los beneficios que éstas generan (Chesher \& Skok, 2000). Así mismo, muchas Pymes ven los costos de las TIC como una barrera para el uso y adopción de e-commerce y negocios en línea (Wymer \& Regan, 2005).

También, Arendt (2008) encontró que aunque las barreras para la aplicación de las TIC en las Pymes varían según el sector económico y el país, existen unas barreras comunes a todas las compañías, dentro de las cuales se encuentran los factores de costos que incluyen costos de equipos y redes de TIC, costos 
de software y reorganización y costos operativos. Por los argumentos anteriores, formulamos la siguiente hipótesis:

H10. Existe una relación negativa entre la adopción de TIC en las Pymes y el desbalance entre los costos de implementación frente a los beneficios recibidos de las mismas.

La siguiente sección describe la metodología utilizada en este estudio para contrastar las hipótesis.

\section{Metodología}

\subsection{Muestra y procedimientos}

Para el contraste de las hipótesis se utilizó una base de datos de Confecámaras con información de empresas de Colombia de distintos sectores económicos. Se diseñó y aplicó un cuestionario electrónico a una muestra de 474 Pymes de actividades de industria y servicios, distribuidas tal como se observa en la Tabla 3.

\begin{tabular}{|c|c|r|}
\hline \multirow{2}{*}{ Sector económico } & Servicios & $72 \%$ \\
\cline { 2 - 3 } & Industria & $28 \%$ \\
\hline \multirow{2}{*}{ Tamaño } & Menos de 10 empleados & $65 \%$ \\
\cline { 2 - 3 } & 11 a 200 empleados & $35 \%$ \\
\hline
\end{tabular}

Tabla 3. Caracterización de las empresas participantes

El cuestionario se diseñó a partir de escalas desarrolladas en estudios previos, tal como se explica en la siguiente sección. En un primer momento se desarrolló una prueba piloto con 20 directores de pymes de la ciudad de Medellín y se recibió la retroalimentación de algunos investigadores académicos para verificar la validez de contenido de las escalas, minimizar ambigüedades y adaptar el lenguaje del instrumento al contexto de las pymes colombianas. Posteriormente, se aplicó el cuestionario virtual ajustado y se realizó el procesamiento estadístico de la información recolectada con en el software de análisis de datos Stata versión 11.2. A través de un análisis factorial de componentes principales se verificó la validez y fiabilidad de las escalas y a continuación se desarrolló un análisis de modelos de regresión múltiple para contrastar las hipótesis propuestas. 


\section{Medidas}

\subsection{Variable dependiente}

Para medir la variable dependiente Adopción de TIC se adaptó una escala a partir de los trabajos de Lucchetti y Sterlacchini (2004) y de Bayo-Moriones y Lera-López (2007), utilizando una escala de 5 puntos con respecto al uso por parte de la empresa de una serie de tecnologías de información y comunicaciones (5: La usa actualmente, 4: Planea usarla en el próximo año, 3: Planea usarla después de un año, 2: No planea usarla, 1: No la conoce). Las tecnologías consideradas son computadores personales (estaciones de trabajo o terminales de cómputo), email de la empresa, red de área local LAN o red de área extendida WAN, Internet, intranet, página web de la empresa, redes sociales empresariales y comercio electrónico (e-commerce).

Para medir la adopción de TIC se consideró una escala compuesta por todas las tecnologías anteriores, pero en los modelos de regresión también se consideró como variable dependiente la adopción individual de cada una de estas tecnologías.

\subsection{Variables independientes}

Las escalas de medición para las variables independientes se obtuvieron de la literatura y estudios previos de adopción de TIC revisados, en los cuales la validez y adecuación de los mismos ya había sido probada. Las diez variables independientes usadas en este estudio están compuestas por escalas de Likert de 5 puntos (5: Totalmente de acuerdo, 4: Parcialmente de acuerdo, 3: Ni de acuerdo, ni en desacuerdo, 2: Parcialmente en desacuerdo, 1: Totalmente en desacuerdo). Los ítems elegidos para medir los constructos de ventaja relativa, observabilidad y complejidad se basaron en los trabajos de Ghobakhloo, Arias-Aranda y Benitez-Amado (2011) y de Ntemana y Olatokun (2012). Para ello, se tradujeron al español los ítems utilizados por dichos autores y con base en la prueba piloto se seleccionaron los ítems más representativos y comprensibles para el público objetivo, teniendo en cuenta las limitaciones de extensión que podía tener el cuestionario, de tal forma que no se afectara la disposición para responder por parte de los encuestados.

Los ítems elegidos para medir los constructos de beneficios (nuevas oportunidades de negocio, comunicación efectiva con clientes, reducción de los costos de negocio, incentivos gubernamentales) y barreras (TIC inadecuadas para el negocio, falta de confianza en la seguridad, desbalance entre costos y 
beneficios de las TIC) se basaron en los trabajos de Thong y Yap (1995), Premkumar (2003), Grandon y Pearson (2004), Saffu, Walker y Hinson (2007) y Al-Qirim (2006). Al igual que con las escalas anteriores, se tradujeron al español y se seleccionaron los ítems más representativos y comprensibles con base en la prueba piloto realizada.

Para garantizar la fiabilidad y validez de las escalas se analizó el Alpha de Cronbach de cada constructo y se realizó un análisis factorial de componentes principales (PCA) para asegurar la validez convergente y discriminante de éstas (Hair, Anderson, Tatham \& Black, 1999).

En la Tabla 4 se presentan los ítems utilizados y el análisis de fiabilidad de las escalas. Puede observarse que casi todas las escalas tienen un Alpha de Cronbach superior a 0.7, tal como lo recomiendan Nunnally y Bernstein (1994). Aun así, las escalas de Complejidad, Comunicación efectiva con los clientes, Incentivos gubernamentales y Desbalance entre costos y beneficios de las TIC presentaron valores inferiores a 0.7. En dichos casos, se eliminaron los ítems correspondientes a las variables COJ3, CEF3, IGO3 y DES3, con lo cual, los Alphas de Cronbach de estas escalas se incrementan a 0.852; $0.771 ; 0.869$ y 0.708 respectivamente.

\begin{tabular}{|c|c|c|c|c|}
\hline Constructo & $\begin{array}{l}\text { Código } \\
\text { Variable }\end{array}$ & Item & $\begin{array}{c}\text { Alpha de } \\
\text { Cronbach } \\
\text { excluyendo la } \\
\text { variable }\end{array}$ & $\begin{array}{c}\text { Alpha de } \\
\text { Cronbach de la } \\
\text { escala }\end{array}$ \\
\hline \multirow{3}{*}{$\begin{array}{l}\text { Ventaja } \\
\text { relativa (REL) }\end{array}$} & REL1 & $\begin{array}{l}\text { Cuando usamos las TIC mejora la eficiencia en } \\
\text { mi empresa }\end{array}$ & 0.830 & \multirow{3}{*}{0.855} \\
\hline & REL2 & $\begin{array}{l}\text { Hay suficientes ventajas en las TIC para } \\
\text { considerar el uso de ellas en la empresa }\end{array}$ & 0.810 & \\
\hline & REL3 & $\begin{array}{l}\text { Las TIC nos ayudan a gestionar mejor el tiempo } \\
\text { en la empresa }\end{array}$ & 0.750 & \\
\hline \multirow{3}{*}{$\begin{array}{l}\text { Observabilidad } \\
\text { (OBS) }\end{array}$} & OBS1 & $\begin{array}{l}\text { Fuimos influenciados por lo que observamos } \\
\text { como beneficios del uso de TIC para la empresa }\end{array}$ & 0.749 & \multirow{3}{*}{0.784} \\
\hline & OBS2 & $\begin{array}{l}\text { Observamos a otras empresas usando las TIC y } \\
\text { vimos las ventajas de usarlas en la empresa }\end{array}$ & 0.624 & \\
\hline & OBS3 & $\begin{array}{l}\text { Antes de usar las TIC en la empresa, hemos } \\
\text { observado a otras empresas cómo las usan }\end{array}$ & 0.743 & \\
\hline \multirow{3}{*}{$\begin{array}{l}\text { Complejidad } \\
\text { (COJ) }\end{array}$} & COJ1 & $\begin{array}{l}\text { Las TIC son difíciles de entender y utilizar en la } \\
\text { empresa }\end{array}$ & 0.395 & \multirow{3}{*}{0.667} \\
\hline & COJ2 & Las TIC son confusas en la empresa & 0.350 & \\
\hline & $\mathrm{COJ} 3$ (**) $^{* *}$ & $\begin{array}{l}\text { No es complicado aprender a usar las TIC en la empresa } \\
(*)\end{array}$ & 0.852 & \\
\hline \multirow{3}{*}{$\begin{array}{l}\text { Nuevas } \\
\text { oportunidades } \\
\text { de negocios } \\
\text { (NOP) }\end{array}$} & NOP1 & $\begin{array}{l}\text { Las TIC le han permitido a la empresa aumentar } \\
\text { el radio de cobertura y llegar a nuevos mercados }\end{array}$ & 0.783 & \multirow{3}{*}{0.860} \\
\hline & NOP2 & $\begin{array}{l}\text { Las TIC le han permitido a la empresa } \\
\text { incursionar en nuevos modelos de negocio }\end{array}$ & 0.797 & \\
\hline & NOP3 & $\begin{array}{l}\text { Por medio de las TIC mi empresa ha podido } \\
\text { conectarse con nuevos proveedores }\end{array}$ & 0.831 & \\
\hline
\end{tabular}




\begin{tabular}{|c|c|c|c|c|}
\hline Constructo & $\begin{array}{l}\text { Código } \\
\text { Variable }\end{array}$ & Item & $\begin{array}{l}\text { Alpha de } \\
\text { Cronbach } \\
\text { excluyendo la } \\
\text { variable }\end{array}$ & $\begin{array}{l}\text { Alpha de } \\
\text { Cronbach de la } \\
\text { escala }\end{array}$ \\
\hline \multirow{3}{*}{$\begin{array}{l}\text { Comunicación } \\
\text { efectiva con los } \\
\text { clientes (CEF) }\end{array}$} & CEF1 & $\begin{array}{l}\text { En la empresa no usamos las TIC para } \\
\text { comunicarnos con los clientes }\left(^{*}\right)\end{array}$ & 0.500 & \multirow{3}{*}{0.669} \\
\hline & CEF2 & $\begin{array}{l}\text { Preferimos usar los medios tradicionales en vez } \\
\text { de las TIC para comunicarnos con los clientes }\left(^{*}\right)\end{array}$ & 0.396 & \\
\hline & $\mathrm{CEF}$ (**) & $\begin{array}{l}\text { Por medio de las TIC mi empresa puede comunicarse más } \\
\text { efectivamente con los clientes }\end{array}$ & 0.771 & \\
\hline \multirow{3}{*}{$\begin{array}{l}\text { Reducción de } \\
\text { los costos de } \\
\text { negocio ( } \mathrm{RCO} \text { ) }\end{array}$} & RCO1 & $\begin{array}{l}\text { Por medio del uso de las TIC en la empresa } \\
\text { hemos logrado disminuir los costos de operación }\end{array}$ & 0.873 & \multirow{3}{*}{0.848} \\
\hline & RCO2 & $\begin{array}{l}\text { Después de implementadas las soluciones de TIC } \\
\text { en la empresa, los procesos se agilizaron }\end{array}$ & 0.703 & \\
\hline & $\mathrm{RCO} 3$ & $\begin{array}{l}\text { La empresa es más productiva con la } \\
\text { incorporación de las TIC en los procesos clave }\end{array}$ & 0.775 & \\
\hline \multirow{3}{*}{$\begin{array}{l}\text { Incentivos } \\
\text { gubernamental } \\
\text { es (IGO) }\end{array}$} & IGO1 & $\begin{array}{l}\text { En la empresa implementamos las TIC a raíz de } \\
\text { una iniciativa de apoyo del gobierno }\end{array}$ & 0.132 & \multirow{3}{*}{0.544} \\
\hline & IGO2 & $\begin{array}{l}\text { Si no hubiera sido por los incentivos } \\
\text { gubernamentales, en la empresa no hubiéramos } \\
\text { adoptado las TIC }\end{array}$ & 0.027 & \\
\hline & IGO3 (**) & $\begin{array}{l}\text { Los incentivos del gobierno no son efectivos para ayudar a } \\
\text { las empresas a adoptar las TIC (*) }\end{array}$ & 0.869 & \\
\hline \multirow{3}{*}{$\begin{array}{l}\text { TIC } \\
\text { inadecuadas } \\
\text { para el negocio } \\
\text { (INA) }\end{array}$} & INA1 & $\begin{array}{l}\text { Nos preocupa que las soluciones de TIC en la } \\
\text { empresa no se puedan aprovechar al máximo }\end{array}$ & 0.768 & \multirow{3}{*}{0.759} \\
\hline & INA2 & $\begin{array}{l}\text { En la empresa nos preocupa que las TIC se } \\
\text { queden obsoletas muy rápidamente }\end{array}$ & 0.586 & \\
\hline & INA3 & $\begin{array}{l}\text { Nos preocupa que las TIC en la empresa se } \\
\text { tengan que personalizar para que funcionen bien }\end{array}$ & 0.666 & \\
\hline \multirow{3}{*}{$\begin{array}{l}\text { Falta de } \\
\text { confianza en la } \\
\text { seguridad } \\
\text { (FCO) }\end{array}$} & FCO1 & $\begin{array}{l}\text { Nos preocupa que las TIC no sean seguras y } \\
\text { confiables para gestionar la información de la } \\
\text { empresa }\end{array}$ & 0.556 & \multirow{3}{*}{0.727} \\
\hline & FCO2 & $\begin{array}{l}\text { Nos preocupa que personas ajenas a la empresa } \\
\text { tengan acceso a la información digital sin permiso }\end{array}$ & 0.521 & \\
\hline & FCO3 & $\begin{array}{l}\text { No nos gusta que la información de la empresa } \\
\text { salga de su sede física }\end{array}$ & 0.805 & \\
\hline \multirow{3}{*}{$\begin{array}{l}\text { Desbalance } \\
\text { entre costos y } \\
\text { beneficios de } \\
\text { las TIC (DES) }\end{array}$} & DES1 & $\begin{array}{l}\text { Nos preocupa que los beneficios de las TIC en la } \\
\text { empresa, no alcancen a cubrir la inversión } \\
\text { realizada }\end{array}$ & 0.407 & \multirow{3}{*}{0.642} \\
\hline & DES2 & $\begin{array}{l}\text { Es una preocupación en la empresa, los costos } \\
\text { futuros para mantener y actualizar las TIC }\end{array}$ & 0.482 & \\
\hline & DES3 (**) & $\begin{array}{l}\text { Antes de implementar un proyecto de TIC en la empresa, } \\
\text { evaluamos la relación beneficio/ costo }\end{array}$ & 0.708 & \\
\hline
\end{tabular}

(*) Escala invertida

$(* *)$ Variable no incluida en los modelos para obtener mejor fiabilidad

Tabla 4. Análisis de fiabilidad de las escalas

Al realizar el análisis de componentes principales, se identificó inicialmente una medida de adecuación muestral KMO de 0.838; lo cual indica que la muestra es adecuada para la realización de este tipo de análisis factoriales. En la Tabla 5 se presentan las cargas factoriales obtenidas para cada ítem con los componentes rotados, aplicando una rotación varimax. Siguiendo un criterio a priori para la extracción de componentes (Hair et al., 1999), se definieron diez factores a extraer, correspondientes al número de constructos propuestos en el marco teórico. Los diez factores extraídos logran explicar el 77.74 por ciento de la varianza de los ítems originales. 
Debido a que este estudio involucra un tamaño de muestra con más de 350 observaciones, se considera significativa una carga factorial mayor a 0.3 para aceptar la validez convergente de cada ítem en su constructo correspondiente (Hair et al., 1999). De igual forma, se espera que la carga factorial de cada ítem sea cercana a cero con respecto a los demás constructos para asegurar su validez discriminante. En la Tabla 5 se observa que las cargas factoriales satisfacen dichos criterios.

\begin{tabular}{|c|c|c|c|c|c|c|c|c|c|c|c|}
\hline \multirow{2}{*}{ Constructos } & \multirow{2}{*}{ Variable } & \multicolumn{10}{|c|}{ Factores } \\
\hline & & 1 & 2 & 3 & 4 & 5 & 6 & 7 & 8 & 9 & 10 \\
\hline \multirow{3}{*}{ Ventaja relativa (REL) } & & 0.577 & 0.041 & 0.028 & 0.024 & -0.029 & 0.059 & 0.000 & 0.031 & 0.001 & 0.01 \\
\hline & & 0.556 & -0.014 & 0.016 & -0.041 & 0.018 & 0.051 & -0.034 & 0.034 & -0.023 & -0.026 \\
\hline & & 0.587 & 0.023 & 0.017 & 0.009 & 0.000 & 0.020 & 0.022 & -0.026 & 0.016 & 0.031 \\
\hline \multirow{3}{*}{ Observabilidad (OBS) } & & .033 & 0.543 & 0.077 & -0.097 & 0.015 & 0.143 & -0.037 & 0.108 & -0.069 & -0.107 \\
\hline & & 0.028 & 0.599 & -0.045 & 0.047 & -0.036 & -0.043 & 0.001 & -0.040 & 0.095 & -0.045 \\
\hline & & 0.000 & 0.574 & -0.032 & 0.028 & 0.009 & -0.058 & 0.043 & -0.081 & -0.084 & 0.142 \\
\hline \multirow{2}{*}{ Complejidad (COJ) } & & 0.010 & -0.008 & 0.709 & 0.001 & 0.032 & -0.004 & 0.025 & 0.028 & -0.011 & -0.035 \\
\hline & & .010 & & 0.655 & 0.009 & -0.078 & & 0.001 & & & 0.066 \\
\hline \multirow{3}{*}{$\begin{array}{l}\text { Nuevas oportunidades de } \\
\text { negocios (NOP) }\end{array}$} & & 0.003 & 0.028 & 0.036 & 0.606 & 0.024 & -0.039 & -0.024 & 0.026 & -0.074 & -0.008 \\
\hline & & 0.009 & -0.039 & -0.016 & 0.569 & -0.031 & & 0.017 & -0.063 & -0.063 & 0.093 \\
\hline & & & & & 0.523 & & & & & & -0.133 \\
\hline \multirow{2}{*}{$\begin{array}{l}\text { Comunicación efectiva con } \\
\text { los clientes (CEF) }\end{array}$} & & 022 & .023 & 0.004 & 0.032 & 0.689 & -0.047 & -0.023 & 0.053 & -0.043 & -0.021 \\
\hline & & 0.015 & 0.013 & -0.038 & -0.035 & 0.672 & 0.075 & 0.037 & -0.051 & -0.013 & 0.061 \\
\hline \multirow{3}{*}{$\begin{array}{l}\text { Reducción de los costos de } \\
\text { negocio (RCO) }\end{array}$} & & -0.007 & 0.000 & -0.097 & 0.070 & -0.047 & 0.490 & 0.070 & -0.076 & & \\
\hline & & 0.004 & -0.017 & 0.018 & -0.002 & -0.004 & 0.593 & -0.031 & 0.024 & 0.099 & -0.031 \\
\hline & & 0.009 & & 0.066 & -0.013 & 0.079 & 0.558 & -0.024 & & 0.020 & -0.056 \\
\hline \multirow{2}{*}{$\begin{array}{l}\text { Incentivos gubernamentales } \\
\text { (IGO) }\end{array}$} & & & & & 0.018 & -0.018 & & & & .003 & -0.024 \\
\hline & & 004 & & 0.002 & -0.022 & 0.026 & -0.008 & 0.708 & -0.002 & 0.022 & 0.016 \\
\hline \multirow{3}{*}{$\begin{array}{l}\text { TIC inadecuadas para el } \\
\text { negocio (INA) }\end{array}$} & & 0.053 & -0.018 & -0.011 & 0.019 & 0.048 & -0.014 & 0.059 & 0.670 & -0.058 & -0.135 \\
\hline & & 0.066 & -0.033 & 0.015 & -0.061 & -0.080 & 0.108 & -0.059 & 0.486 & -0.077 & 0.165 \\
\hline & & -0.033 & 0.007 & -0.140 & 0.017 & -0.152 & 0.006 & 0.047 & 0.363 & -0.060 & 0.239 \\
\hline \multirow{3}{*}{$\begin{array}{l}\text { Falta de confianza en la } \\
\text { seguridad (FCO) }\end{array}$} & & -0.031 & 0.073 & 0.118 & 0.050 & 0.063 & -0.083 & -0.090 & 0.201 & 0.306 & 0.188 \\
\hline & & -0.005 & 0.059 & 0.054 & 0.045 & 0.105 & -0.153 & -0.036 & 0.283 & 0.429 & -0.022 \\
\hline & FCO3 & 0.001 & -0.026 & -0.042 & -0.036 & -0.056 & 0.071 & 0.028 & -0.089 & 0.786 & -0.019 \\
\hline \multirow{2}{*}{$\begin{array}{l}\text { Desbalance entre costos y } \\
\text { beneficios de las TIC (DES) }\end{array}$} & & 0.016 & -0.007 & 0.033 & 0.032 & 0.048 & -0.062 & 0.021 & -0.083 & 0.191 & 0.550 \\
\hline & DES2 & 0.012 & 0.000 & 0.004 & -0.028 & 0.016 & 0.032 & -0.017 & -0.007 & -0.116 & 0.679 \\
\hline
\end{tabular}

Matriz de componentes rotados. Método: Componentes principales con rotación Varimax

Tabla 5. Análisis de componentes principales

\section{Análisis y Resultados}

Los resultados del análisis VIF (Variance Inflation Factor) presentado en la Tabla 6, muestran que para todos los factores, la tolerancia es mayor a 0.1 y los valores de inflación de la varianza VIF son menores que diez (varían desde 1.29 hasta 1.92), lo cual indica que no hay problemas de multicolinealidad entre las variables independientes para el desarrollo posterior de los análisis de regresión múltiple (Hair et al., 1999). 


\begin{tabular}{|l|r|r|}
\hline Variables independientes & Tolerancia & VIF \\
\hline Ventaja relativa (REL) & 0.680783 & 1.47 \\
\hline Observabilidad (OBS) & 0.778189 & 1.29 \\
\hline Complejidad (COJ) & 0.601220 & 1.66 \\
\hline Nuevas oportunidades de negocios (NOP) & 0.602993 & 1.66 \\
\hline Comunicación efectiva con los clientes (CEF) & 0.641279 & 1.56 \\
\hline Reducción de los costos de negocio (RCO) & 0.525241 & 1.90 \\
\hline Incentivos gubernamentales (IGO) & 0.691356 & 1.45 \\
\hline TIC inadecuadas para el negocio (INA) & 0.573429 & 1.74 \\
\hline Falta de confianza en la seguridad (FCO) & 0.542718 & 1.84 \\
\hline Desbalance entre costos y beneficios de las TIC (DES) & 0.521501 & 1.92 \\
\hline
\end{tabular}

Tabla 6. Resultados de análisis de multicolinealidad de las variables

En la Tabla 7 se presentan los principales estadísticos descriptivos de las variables compuestas, tales como la media, la desviación estándar y las correlaciones entre las diferentes variables del modelo. Se destaca que los constructos que presentan una media más alta son la ventaja relativa, los beneficios de nuevas oportunidades de negocios, reducción de los costos para el negocio y comunicaciones efectivas con los clientes, y las barreras asociadas a la falta de confianza en la seguridad y las TIC consideradas inadecuadas para el negocio.

\begin{tabular}{|c|c|c|c|c|c|c|c|c|c|c|c|c|c|c|c|}
\hline \multicolumn{2}{|r|}{ Variable } & Tipo & Media & DE & 1 & 2 & 3 & 4 & 5 & 6 & 7 & 8 & 9 & 10 & 11 \\
\hline 1 & Adop'TICs & DV & 3.28 & 0.513 & 1.00 & & & & & & & & & & \\
\hline 2 & REL & IV & 4.70 & 0.523 & $0.23 *$ & 1.00 & & & & & & & & & \\
\hline 3 & OBS & IV & 3.52 & 0.967 & $0.09 *$ & $0.21 *$ & 1.00 & & & & & & & & \\
\hline 4 & $\mathrm{COJ}$ & IV & 2.05 & 0.978 & $-0.08 *$ & $-0.21 *$ & $0.28 *$ & 1.00 & & & & & & & \\
\hline 5 & NOP & IV & 4.15 & 0.773 & $0.43^{*}$ & $0.43 *$ & $0.21 *$ & $-0.15 *$ & 1.00 & & & & & & \\
\hline 6 & CEF & IV & 3.96 & 1.050 & $0.09 *$ & $0.21 *$ & $-0.10 *$ & $-0.49 *$ & $0.15 *$ & 1.00 & & & & & \\
\hline 7 & RCO & IV & 4.12 & 0.728 & $0.31 *$ & $0.51 *$ & $0.23 *$ & $-0.21 *$ & $0.61 *$ & $0.20 *$ & 1.00 & & & & \\
\hline 8 & IGO & IV & 1.82 & 0.975 & 0.02 & $-0.12 *$ & $0.20 *$ & $0.44 *$ & -0.00 & $-0.47 *$ & -0.03 & 1.00 & & & \\
\hline 9 & INA & IV & 3.45 & 0.903 & -0.07 & -0.04 & $0.11 *$ & $0.22 *$ & -0.07 & $-0.25 *$ & $-0.10 *$ & $0.14^{*}$ & 1.00 & & \\
\hline 10 & FCO & IV & 3.53 & 0.947 & $-0.15^{*}$ & -0.01 & $0.22 *$ & $0.31 *$ & $-0.08 *$ & $-0.23 *$ & $-0.09 *$ & $0.11 *$ & $0.56^{*}$ & 1.00 & \\
\hline 11 & DES & IV & 3.27 & 1.018 & -0.03 & -0.07 & $0.14 *$ & $0.28 *$ & $-0.08 *$ & $-0.25 *$ & $-0.17 *$ & $0.16^{*}$ & $0.60 *$ & $0.60 *$ & 1.00 \\
\hline
\end{tabular}

Notas. VD: Variable dependiente; VI: Variable independiente; DE: Desviación estándar; ${ }^{*} \mathrm{p}<0.10$

Tabla 7. Estadísticos descriptivos y correlaciones

Los resultados del análisis de regresión múltiple entre las variables independientes que miden las tres características del modelo de difusión de innovación (DOI), los beneficios, y las barreras y la variable dependiente de adopción de TIC, se presentan en la Tabla 8. En dicha tabla se muestra la regresión con la variable "Adopción de TIC" compuesta por la media de las ocho tecnologías medidas (PCs, e-mail, LAN/WAN, Internet, Intranet, Web Page, Redes Sociales y e-commerce), y también con cada una de estas TIC de forma individual. 
En el modelo compuesto, hay tres variables (un beneficio y dos barreras) que están significativamente asociadas con la adopción de TIC por parte de las Pymes en Colombia. El beneficio percibido de las TIC es que permiten crear nuevas oportunidades de negocios. Sin embargo, la falta de confianza en la seguridad de TIC y la percepción de que la inversión en TIC no retorna los beneficios esperados para los negocios (Desbalance entre costos y beneficios), se convierten en barreras que se asocian negativamente con la adopción de TIC. Al encontrar en el modelo compuesto éstas relaciones estadísticamente significativas, se aceptan las hipótesis H4, H9 y H10.

A su vez, para el modelo compuesto, ninguna de las tres variables independientes asociadas al modelo DOI, está significativamente asociada con la adopción de TIC en las Pymes. Por lo tanto, H1, H2 y H3 se rechazan. Igualmente, la comunicación efectiva con los clientes, la reducción de los costos de negocio, los incentivos gubernamentales y las TIC inadecuadas para el negocio, no predicen significativamente la adopción de TIC, por lo tanto H5, H6, H7 y H8 se rechazan.

\begin{tabular}{|c|c|c|c|c|c|c|c|c|c|}
\hline Modelo & 1 & 2 & 3 & 4 & 5 & 6 & 7 & 8 & 9 \\
\hline V. Dep. & $\begin{array}{c}\text { Adop } \\
\text { TIC }\end{array}$ & PCs & Email & LAN & Internet & Intranet & $\begin{array}{l}\text { Página } \\
\text { Web }\end{array}$ & $\begin{array}{c}\text { Redes } \\
\text { Sociales }\end{array}$ & $\begin{array}{l}\text { Comercio } \\
\text { electrónico }\end{array}$ \\
\hline REL & $\begin{array}{r}0.049 \\
(1.01)\end{array}$ & $\begin{array}{r}0.106^{* *} \\
(2.45)\end{array}$ & $\begin{array}{r}0.145^{* * *} \\
(3.38)\end{array}$ & $\begin{array}{c}0.214 \\
(1.60)\end{array}$ & $\begin{array}{r}0.091 * * \\
(2.24)\end{array}$ & $\begin{array}{l}-0.027 \\
(-0.18)\end{array}$ & $\begin{array}{c}0.036 \\
(0.38)\end{array}$ & $\begin{array}{c}0.096 \\
(0.79)\end{array}$ & $\begin{array}{r}-0.268^{*} \\
(-1.94)\end{array}$ \\
\hline OBS & $\begin{array}{l}0.004 \\
(0.15)\end{array}$ & $\begin{array}{l}-0.017 \\
-0.80)\end{array}$ & $\begin{array}{l}-0.021 \\
(-0.97)\end{array}$ & $\begin{array}{l}-0.087 \\
-1.28)\end{array}$ & $\begin{array}{l}0.006 \\
(0.29)\end{array}$ & $\begin{array}{l}0.033 \\
(0.44)\end{array}$ & $\begin{array}{l}-0.006 \\
(-0.12)\end{array}$ & $\begin{array}{l}0.095 \\
(1.54)\end{array}$ & $\begin{array}{l}0.028 \\
(0.40)\end{array}$ \\
\hline $\mathrm{COJ}$ & $\begin{array}{l}0.005 \\
(0.18)\end{array}$ & $\begin{array}{l}-0.000 \\
(-0.01)\end{array}$ & $\begin{array}{r}-0.065^{* * *} \\
(-2.67)\end{array}$ & $\begin{array}{l}-0.024 \\
(-0.31)\end{array}$ & $\begin{array}{l}-0.013 \\
(-0.55)\end{array}$ & $\begin{array}{l}-0.002 \\
(-0.03)\end{array}$ & $\begin{array}{r}0.055 \\
(1.02)\end{array}$ & $\begin{array}{l}0.011 \\
(0.16)\end{array}$ & $\begin{array}{l}0.079 \\
(1.00)\end{array}$ \\
\hline NOP & $\begin{array}{r}0.237 * * * \\
(6.72)\end{array}$ & $\begin{array}{l}0.030 \\
(0.97)\end{array}$ & $\begin{array}{l}0.044 \\
(1.42)\end{array}$ & $\begin{array}{r}0.159 \\
(1.65)\end{array}$ & $\begin{array}{l}0.020 \\
(0.69)\end{array}$ & $\begin{array}{r}0.305^{* * *} \\
(2.89)\end{array}$ & $\begin{array}{r}0.467 * * * \\
(6.94)\end{array}$ & $\begin{array}{r}0.463 * * * \\
(5.31)\end{array}$ & $\begin{array}{r}0.404 * * * \\
(4.07)\end{array}$ \\
\hline CEF & $\begin{array}{l}0.008 \\
(0.33)\end{array}$ & $\begin{array}{l}0.017 \\
(0.78)\end{array}$ & $\begin{array}{l}0.007 \\
(0.31)\end{array}$ & $\begin{array}{l}0.037 \\
(0.54)\end{array}$ & $\begin{array}{c}0.026 \\
(1.26)\end{array}$ & $\begin{array}{l}-0.105 \\
(-1.39)\end{array}$ & $\begin{array}{r}0.063 \\
(1.31)\end{array}$ & $\begin{array}{l}-0.018 \\
(-0.29)\end{array}$ & $\begin{array}{l}0.039 \\
(0.56)\end{array}$ \\
\hline RCO & $\begin{array}{c}0.048 \\
(1.20)\end{array}$ & $\begin{array}{l}0.007 \\
(0.20)\end{array}$ & $\begin{array}{l}0.020 \\
(0.58)\end{array}$ & $\begin{array}{l}0.158 \\
(1.43)\end{array}$ & $\begin{array}{l}-0.030 \\
(-0.91)\end{array}$ & $\begin{array}{r}0.205^{*} \\
(1.71)\end{array}$ & $\begin{array}{l}-0.065 \\
-0.85)\end{array}$ & $\begin{array}{l}-0.085 \\
-0.85)\end{array}$ & $\begin{array}{l}0.174 \\
(1.74)\end{array}$ \\
\hline IGO & $\begin{array}{l}0.019 \\
(0.72)\end{array}$ & $\begin{array}{r}0.008 \\
(0.33)\end{array}$ & $\begin{array}{l}0.010 \\
(0.42)\end{array}$ & $\begin{array}{c}0.048 \\
(0.67)\end{array}$ & $\begin{array}{l}-0.020 \\
(-0.92)\end{array}$ & $\begin{array}{l}0.075 \\
(0.96)\end{array}$ & $\begin{array}{l}-0.045 \\
(-0.89)\end{array}$ & $\begin{array}{l}-0.010 \\
(-0.16)\end{array}$ & $\begin{array}{l}0.084 \\
(1.14)\end{array}$ \\
\hline INA & $\begin{array}{l}-0.013 \\
(-0.43)\end{array}$ & $\begin{array}{r}0.029 \\
(1.05)\end{array}$ & $\begin{array}{l}-0.026 \\
(-0.96)\end{array}$ & $\begin{array}{l}-0.081 \\
(-0.95)\end{array}$ & $\begin{array}{c}0.004 \\
(0.17)\end{array}$ & $\begin{array}{l}-0.055 \\
(-0.60)\end{array}$ & $\begin{array}{l}0.046 \\
(0.78)\end{array}$ & $\begin{array}{l}-0.056 \\
(-0.73)\end{array}$ & $\begin{array}{l}0.031 \\
(0.36)\end{array}$ \\
\hline FCO & $\begin{array}{r}-0.102^{* * *} \\
(-3.35)\end{array}$ & $\begin{array}{r}0.002 \\
(0.08)\end{array}$ & $\begin{array}{l}-0.025 \\
(-0.94)\end{array}$ & $\begin{array}{l}-0.061 \\
(-0.74)\end{array}$ & $\begin{array}{l}-0.001 \\
(-0.04)\end{array}$ & $\begin{array}{r}-0.335^{* * *} \\
(-3.70)\end{array}$ & $\begin{array}{r}-0.139 * * \\
(-2.41)\end{array}$ & $\begin{array}{l}-0.081 \\
-1.08)\end{array}$ & $\begin{array}{r}-0.171^{* *} \\
(-2.00)\end{array}$ \\
\hline DES & $\begin{array}{r}0.070^{* *} \\
(2.44)\end{array}$ & $\begin{array}{l}-0.018 \\
(-0.70) \\
\end{array}$ & $\begin{array}{r}0.090^{* * *} \\
(3.59)\end{array}$ & $\begin{array}{r}0.150^{*} \\
(1.90)\end{array}$ & $\begin{array}{l}0.005 \\
(0.21)\end{array}$ & $\begin{array}{r}0.147^{*} \\
(1.71)\end{array}$ & $\begin{array}{c}0.078 \\
(1.42)\end{array}$ & $0.072(1.02)$ & $\begin{array}{l}0.035 \\
(0.44)\end{array}$ \\
\hline$n s$ & $\begin{array}{r}1.956^{* * *} \\
(7.58)\end{array}$ & $\begin{array}{r}3.209^{* * *} \\
(14.02)\end{array}$ & $\begin{array}{r}3.003^{* * *} \\
(13.27)\end{array}$ & $\begin{array}{c}1.110 \\
(1.57)\end{array}$ & $\begin{array}{r}3.473^{* * * *} \\
(16.21)\end{array}$ & $\begin{array}{r}1.391^{*} \\
(1.80)\end{array}$ & $\begin{array}{r}1.395^{* * *} \\
(2.83)\end{array}$ & $\begin{array}{r}1.073^{*} \\
(1.68)\end{array}$ & $\begin{array}{l}0.997 \\
(1.37)\end{array}$ \\
\hline $\mathbf{N}$ & 474 & 474 & 474 & 474 & 474 & 474 & 474 & 474 & 474 \\
\hline R-sq & 0.212 & 0.036 & 0.112 & 0.057 & 0.035 & 0.100 & 0.151 & 0.103 & 0.090 \\
\hline adj. R-sq & 0.195 & 0.015 & 0.093 & 0.036 & 0.14 & 0.080 & 0.132 & 0.084 & 0.070 \\
\hline $\mathbf{F}$ & $12.49 * * *$ & $1.73 *$ & $5.83^{* * *}$ & $2.78^{* * *}$ & $1.68^{*}$ & $5.14 * * *$ & $8.22 * * *$ & $5.32 * * *$ & $4.56^{* * *}$ \\
\hline
\end{tabular}

Notas. Estadístico t en paréntesis; ${ }^{*} \mathrm{p}<0.1 ;{ }^{* *} \mathrm{p}<0.05$; ${ }^{* * *} \mathrm{p}<0.01$

Tabla 8. Modelos de regresión múltiple por tipo de TIC 
Ahora, cuando se revisan los resultados estadísticamente significativos de adopción de TIC en los modelos individuales de cada una de las tecnologías que se muestran en la Tabla 8 , se encuentra que la adopción de tecnologías como los PCs, el email de la empresa, y el internet, están positivamente relacionados con la ventaja relativa del modelo DOI, y el comercio electrónico se asocia negativamente. De igual forma, la adopción del email de la empresa está negativamente asociada con el factor de complejidad del modelo DOI.

Por otro lado, la fuerte relación positiva en el modelo compuesto entre la adopción de TIC y las nuevas oportunidades de negocio, está altamente influenciada por la adopción de las tecnologías intranet, página web de la empresa, redes sociales y comercio electrónico. También, se encuentra una gran influencia de las tecnologías Intranet, página web de la empresa y comercio electrónico sobre la relación negativa entre la adopción de TIC en el modelo compuesto y la falta de confianza en la seguridad y privacidad de las mismas. Finalmente, las tecnologías que más influyen en la relación encontrada entre la adopción de TIC del modelo compuesto y la percepción de desbalance entre costos y beneficios de las TIC, son el e-mail, las redes LAN y la intranet.

La Tabla 9 compara los resultados del modelo general de regresión múltiple entre las diez variables independientes y la variable dependiente de adopción de TIC, con cuatro modelos adicionales considerando las variables de clasificación "tamaño" y "sector", y otros cuatro modelos aplicando las combinaciones de ambas variables. La variable "tamaño" toma el valor "micro" para las empresas que tienen entre 1 y 10 empleados, y el valor "pyme" para las empresas que están por encima de 10 empleados. La variable "sector", toma los valores "industria" o "servicio".

Al revisar los resultados del modelo para las empresas de servicio en la Tabla 9, se encuentra que cinco de las diez variables independientes del modelo son estadísticamente significativas asociadas a la adopción de TIC, mientras que para las empresas industriales solo una variable influye significativamente en la adopción de las TIC. El factor común para ambos sectores corresponde a las nuevas oportunidades de negocio y los factores adicionales que influyen en las empresas de servicios, son la falta de confianza en la seguridad de TIC, la ventaja relativa, el retorno de los beneficios esperados de la inversión en TIC y la reducción de costos.

Cuando se analiza la dimensión del tamaño de la empresa, se encuentran dos factores comunes que influyen significativamente en la adopción de las TIC: las nuevas oportunidades de negocio y la falta de confianza en la seguridad de las TIC, y solo para las microempresas resulta como un factor influyente en forma significativa, el retorno de los beneficios esperados de la inversión en TIC. 


\begin{tabular}{|c|c|c|c|c|c|c|c|c|c|}
\hline Modelo & 1 & 2 & 3 & 4 & 5 & 6 & 7 & 8 & 9 \\
\hline Muestra & Total & Micro & Pymes & Servicio & Industria & $\begin{array}{c}\text { Micro } \\
\text { Servicio }\end{array}$ & $\begin{array}{c}\text { Micro } \\
\text { Industria }\end{array}$ & $\begin{array}{l}\text { Pymes } \\
\text { Servicio }\end{array}$ & $\begin{array}{c}\text { Pymes } \\
\text { Industria }\end{array}$ \\
\hline REL & $\begin{array}{r}0.049 \\
(1.01)\end{array}$ & $\begin{array}{c}0.096 \\
(1.59) \\
\end{array}$ & $\begin{array}{l}-0.091 \\
(-1.11) \\
\end{array}$ & $\begin{array}{r}0.116^{* *} \\
(2.05)\end{array}$ & $\begin{array}{l}-0.111 \\
(-1.13)\end{array}$ & $\begin{array}{c}0.102 \\
(1.54)\end{array}$ & $\begin{array}{l}-0.002 \\
(-0.01)\end{array}$ & $\begin{array}{l}-0.011 \\
(-0.10)\end{array}$ & $\begin{array}{l}-0.136 \\
(-1.00)\end{array}$ \\
\hline OBS & $\begin{array}{l}0.004 \\
(0.15)\end{array}$ & $\begin{array}{l}0.011 \\
(0.38)\end{array}$ & $\begin{array}{l}0.006 \\
(0.15)\end{array}$ & $\begin{array}{l}0.015 \\
(0.54)\end{array}$ & $\begin{array}{l}0.020 \\
(0.36)\end{array}$ & $\begin{array}{l}0.017 \\
(0.47)\end{array}$ & $\begin{array}{c}0.072 \\
(1.09)\end{array}$ & $\begin{array}{l}0.004 \\
(0.09)\end{array}$ & $\begin{array}{l}-0.049 \\
(-0.43)\end{array}$ \\
\hline $\mathrm{COJ}$ & $\begin{array}{c}0.005 \\
(0.18)\end{array}$ & $\begin{array}{r}-0.022 \\
(-0.61) \\
\end{array}$ & $\begin{array}{c}0.036 \\
(0.84)\end{array}$ & $\begin{array}{l}0.020 \\
(0.63)\end{array}$ & $\begin{array}{l}-0.004 \\
(-0.07)\end{array}$ & $\begin{array}{l}-0.019 \\
(-0.46)\end{array}$ & $\begin{array}{c}-0.033 \\
(-0.41)\end{array}$ & $\begin{array}{l}0.078 \\
(1.62)\end{array}$ & $\begin{array}{r}0.036 \\
(0.39)\end{array}$ \\
\hline NOP & $\begin{array}{r}0.237 * * * \\
(6.72)\end{array}$ & $\begin{array}{r}0.226^{* * *} \\
(5.28)\end{array}$ & $\begin{array}{r}0.220^{* * *} \\
(3.54)\end{array}$ & $\begin{array}{r}0.231^{* * *} \\
(5.90)\end{array}$ & $\begin{array}{r}0.243^{* * *} \\
(3.14)\end{array}$ & $\begin{array}{r}0.235^{* * *} \\
(4.73)\end{array}$ & $\begin{array}{r}0.199 * * \\
(2.29)\end{array}$ & $\begin{array}{r}0.198^{* * *} \\
(3.08)\end{array}$ & $\begin{array}{r}0.390^{* *} \\
(2.35)\end{array}$ \\
\hline CEF & $\begin{array}{l}0.008 \\
(0.33)\end{array}$ & $\begin{array}{c}0.019 \\
(0.59)\end{array}$ & $\begin{array}{l}-0.005 \\
(-0.14)\end{array}$ & $\begin{array}{l}0.032 \\
(1.13)\end{array}$ & $\begin{array}{l}-0.034 \\
(-0.65)\end{array}$ & $\begin{array}{c}0.043 \\
(1.15)\end{array}$ & $\begin{array}{l}-0.048 \\
(-0.67)\end{array}$ & $\begin{array}{l}0.017 \\
(0.40)\end{array}$ & $\begin{array}{l}-0.076 \\
(-0.75)\end{array}$ \\
\hline RCO & $\begin{array}{c}0.048 \\
(1.20)\end{array}$ & & & $\begin{array}{r}0.076^{*} \\
(1.66)\end{array}$ & & $\begin{array}{l}0.076 \\
(1.34)\end{array}$ & $\begin{array}{c}-0.010 \\
(-0.10)\end{array}$ & $\begin{array}{l}0.062 \\
(0.78)\end{array}$ & $\begin{array}{l}-0.163 \\
(-0.98) \\
\end{array}$ \\
\hline IGO & $\begin{array}{l}0.019 \\
(0.72)\end{array}$ & $\begin{array}{l}0.029 \\
(0.89)\end{array}$ & $\begin{array}{l}0.010 \\
(0.23)\end{array}$ & $\begin{array}{l}0.014 \\
(0.48)\end{array}$ & $\begin{array}{l}0.013 \\
(0.23)\end{array}$ & $\begin{array}{l}0.017 \\
(0.45)\end{array}$ & $\begin{array}{l}0.040 \\
(0.57)\end{array}$ & $\begin{array}{l}-0.011 \\
(-0.26)\end{array}$ & $\begin{array}{l}-0.028 \\
(-0.19)\end{array}$ \\
\hline INA & $\begin{array}{l}-0.013 \\
(-0.43)\end{array}$ & $\begin{array}{l}-0.019 \\
(-0.51)\end{array}$ & $\begin{array}{l}0.012 \\
(0.22)\end{array}$ & $\begin{array}{l}0.009 \\
(0.26)\end{array}$ & $\begin{array}{l}-0.054 \\
(-0.84)\end{array}$ & $\begin{array}{l}-0.025 \\
(-0.57)\end{array}$ & $\begin{array}{l}-0.014 \\
(-0.19)\end{array}$ & $\begin{array}{c}0.094 \\
(1.61)\end{array}$ & $\begin{array}{l}-0.214 \\
(-1.62)\end{array}$ \\
\hline FCO & $\begin{array}{r}-0.102^{* * *} \\
(-3.35) \\
\end{array}$ & $\begin{array}{r}-0.085^{* *} \\
(-2.31) \\
\end{array}$ & $\begin{array}{r}-0.128^{* *} \\
(-2.40) \\
\end{array}$ & $\begin{array}{r}-0.130 * * * \\
(-3.81)\end{array}$ & $\begin{array}{l}-0.025 \\
(-0.39)\end{array}$ & $\begin{array}{r}-0.121 * * * \\
(-2.85)\end{array}$ & $\begin{array}{l}0.033 \\
(0.43)\end{array}$ & $\begin{array}{r}-0.121 * * \\
(-2.10) \\
\end{array}$ & $\begin{array}{l}-0.219 \\
(-1.66)\end{array}$ \\
\hline DES & $\begin{array}{r}0.070^{* *} \\
(2.44)\end{array}$ & $\begin{array}{r}0.069^{* *} \\
(2.04)\end{array}$ & $\begin{array}{c}0.052 \\
(0.92)\end{array}$ & $\begin{array}{r}0.078^{* *} \\
(2.46)\end{array}$ & $\begin{array}{l}0.040 \\
(0.64)\end{array}$ & $\begin{array}{r}0.102^{* * *} \\
(2.64)\end{array}$ & $\begin{array}{l}-0.021 \\
(-0.31)\end{array}$ & $\begin{array}{l}0.002 \\
(0.04)\end{array}$ & $\begin{array}{r}0.275^{*} \\
(1.76)\end{array}$ \\
\hline ns & $\begin{array}{r}1.956^{* * *} \\
(7.58)\end{array}$ & $\begin{array}{r}1.653^{* * *} \\
(5.35)\end{array}$ & $\begin{array}{r}3.059 * * * \\
(6.43)\end{array}$ & $\begin{array}{r}1.412^{* * *} \\
(4.71)\end{array}$ & $\begin{array}{r}3.013^{* * *} \\
(5.89)\end{array}$ & $\begin{array}{r}1.428^{* * *} \\
(3.95)\end{array}$ & $\begin{array}{r}2.307 * * * \\
(3.48)\end{array}$ & $\begin{array}{r}2.283^{* * *} \\
(3.94)\end{array}$ & $\begin{array}{r}4.062^{* * *} \\
(3.93)\end{array}$ \\
\hline $\mathbf{N}$ & 474 & 310 & 164 & 341 & 133 & 224 & 86 & 117 & 47 \\
\hline R-sq & 0.212 & 0.255 & 0.136 & 0.291 & 0.113 & 0.334 & 0.134 & 0.193 & 0.266 \\
\hline adj. R-sq & 0.195 & 0.230 & 0.080 & 0.270 & 0.040 & 0.303 & 0.019 & 0.117 & 0.062 \\
\hline $\mathbf{F}$ & $12.49^{* * *}$ & $10.23^{* * *}$ & $2.41 * *$ & $13.57 * * *$ & 1.55 & $10.69^{* * *}$ & 1.16 & $2.54 * * *$ & 1.31 \\
\hline
\end{tabular}

Notas. Variable Dependiente: AdopTIC. Estadístico t en paréntesis; ${ }^{*} \mathrm{p}<0.1 ;{ }^{* *} \mathrm{p}<0.05 ;{ }^{* * *} \mathrm{p}<0.01$

Tabla 9. Modelos de regresión múltiple por tipo de empresa

\section{Discusión y conclusiones}

Ante la escasez de trabajos empíricos que analicen la adopción tecnológica en empresas colombianas, este estudio brinda un valioso aporte de generación de conocimiento al intentar explicar la adopción de TIC por parte de las Pymes en Colombia de manera integral y cuantitativa, con una muestra representativa, que permite cierta generalización de los resultados dentro de la población estudiada.

En este trabajo hemos investigado y analizado el impacto que tienen diez factores sobre la adopción de las TIC por parte de las Pymes colombianas. Para la elaboración de los constructos, se partió del modelo de difusión de innovación de Rogers (1995), seleccionando tres de los cinco constructos definidos en él: ventaja relativa, complejidad y observabilidad; complementados con otros constructos que miden los beneficios y barreras percibidos por las empresas para la adopción de las TIC: nuevas oportunidades de negocios, comunicación efectiva con los clientes, reducción de los costos del negocio, incentivos gubernamentales, las TIC inadecuadas para el negocio, falta de confianza en la seguridad de 
las TIC y el desbalance entre costos y beneficios de las TIC. El modelo diseñado para esta investigación resultante fue probado y validado estadísticamente.

Formulamos diez hipótesis, que fueron probadas empíricamente con los datos de una muestra de 474 microempresas y pymes colombianas, de actividades económicas de industria y servicios.

Los resultados de la investigación muestran que en términos generales, las nuevas oportunidades de negocios parece ser el beneficio más importante que afecta la disposición de las Pymes colombianas para adoptar las TIC y en cuanto a las barreras que limitan la adopción de TIC se resalta la falta de confianza en la seguridad de las TIC y el desbalance entre costos y beneficios de las TIC (que la inversión en TIC no retorne los beneficios esperados).

Cuando se profundiza en el análisis comparando los modelos por el tipo de tecnología, aparecen factores adicionales como la ventaja relativa, que afecta positivamente la disposición de las Pymes colombianas para la adopción del email, los computadores personales, la intranet y el comercio electrónico, así como la complejidad, que afecta negativamente la adopción del email en la empresa.

Al comparar los modelos por sector económico, se encuentra que las empresas del sector servicios son influenciadas por cinco factores para adoptar las TIC: la ventaja relativa, las nuevas oportunidades de negocio, la falta de confianza en la seguridad de las TIC, el retorno de los beneficios esperados de la inversión en TIC y la reducción de costos; mientras que las empresas del sector industria, solo son influenciadas por el factor de nuevas oportunidades de negocio.

La comparación de los modelos por tamaño de empresa, muestra que se comparte la influencia de dos factores para la adopción de las TIC: nuevas oportunidades de negocio y falta de confianza en la seguridad de las TIC, pero para las microempresas también influye el retorno de los beneficios esperados de la inversión en TIC.

Este estudio presenta las limitaciones propias de una investigación de corte transversal, lo cual restringe la interpretación de relaciones de causalidad entre variables independientes y dependientes (tal como se podría hacer con un estudio longitudinal). De igual forma, se considera el hecho de que la generalización de los resultados se circunscribe al contexto geográfico y tipo de empresas encuestadas, y se debe tener en cuenta el hecho de que la calificación de las escalas está basada en las percepciones de los gerentes, en lugar de medidas objetivas. Adicionalmente, debido a la cantidad de constructos introducidos en los modelos y las restricciones de extensión que permitía el cuestionario, se han utilizado escalas con menos ítems que las originales, lo cual puede afectar un poco su validez de 
contenido. Esta limitación podría superarse en futuras investigaciones utilizando las escalas completas identificadas en la literatura.

Si bien los resultados de este estudio no parecen ser contundentes con la confirmación de todas las hipótesis, pueden tener importantes implicaciones para los gerentes de las Pymes, y para los distintos gremios que las abarcan en distintos sectores, así como para el gobierno nacional o local, dado que es importante reforzar el beneficio asociado a la adopción de las TIC, además de empezar a romper las dos barreras encontradas en ésta investigación.

El hecho de que las nuevas oportunidades de negocio se perciban como un habilitador de la adopción de las TIC por parte de las Pymes de Colombia, se constituye en un elemento motivador para que se emprendan programas de educación y apropiación más enfocados en la utilidad de las TIC para el negocio.

Si bien es cierto que la falta de confianza en la seguridad de las TIC es una percepción generalizada en pequeñas, medianas y grandes empresas, se debe combatir principalmente en las Pymes, porque el miedo a la vulnerabilidad de la privacidad de sus datos o a la disponibilidad de su información, inhibe la adopción de estas tecnologías.

Así mismo, deben crearse planes y formas más innovadoras de mostrar las bondades, beneficios y retornos de las inversiones en TIC por parte de las Pymes, porque queda claro que la manera como vendedores de software, proveedores de telecomunicaciones y el gobierno mismo han tratado de vender el potencial de las TIC a las pymes, no es la mejor, dado que subyace entre éstas la idea que la inversión en TIC no retorna beneficios suficientes para sus negocios.

Finalmente, se proponen como futuras líneas de investigación, abarcar el impacto que tiene la adopción de las TIC en la rentabilidad de las empresas, estudios longitudinales (verificar a futuro cómo evoluciona la adopción de TIC en las Pymes y sus factores antecedentes), tener en cuenta la adopción de nuevas TIC (LTE, big data, social media, IoT, etc), o también usar otros modelos de adopción tecnológica como TAM, TRA o TPB, en lugar de DOI para analizar la adopción de las TIC en las Pymes de Colombia. 


\section{Referencias}

Agarwal, R., \& Prasad, J. (1997). The role of innovation characteristics and perceived voluntariness in the acceptance of information technologies. Decision sciences, 28(3), 557-582. http://dx.doi.org/10.1111/j.1540-5915.1997.tb01322.x

Ajzen, I. (1985). From intentions to actions: A theory of planned behavior. In J. Kuhl \& J. Beckman (Eds.), Action-control: From cognition to behavior (pp. 11-39). Berlin Heidelberg: Springer. http://dx.doi.org/10.1007/978-3-642-69746-3_2

Ajzen, I., \& Fishbein, M. (1980). Understanding attitudes and predicting social behaviour. Englewood Cliffs, NJ: Prentice-Hall.

Al-Qirim, N. (2006). Personas of E-Commerce Adoption in Small Businesses in New Zealand. Journal of Electronic Commerce in Organizations, 4(3), 18-45. http://dx.doi.org/10.4018/jeco.2006070102

Aljifri, H.A., Pons, A., \& Collins, D. (2003). Global e-commerce: a framework for understanding and overcoming the trust barrier. Information Management \& Computer Security, 11(3), 130-138. http://dx.doi.org/10.1108/09685220310480417

Alkalbani, S., Rezgui, Y., Vorakulpipat, C., \& Wilson, I.E. (2013). ICT adoption and diffusion in the construction industry of a developing economy: The case of the sultanate of Oman. Architectural Engineering and Design Management, 9(1), 62-75. http://dx.doi.org/10.1080/17452007.2012.718861

Alshamaila, Y., Papagiannidis, S., \& Li, F. (2013). Cloud computing adoption by SMEs in the north east of England: A multi-perspective framework. Journal of Enterprise Information Management, 26(3), 250-275. http://dx.doi.org/10.1108/17410391311325225

APEC - Asia Pacific Economic Cooperation (1999). SME Electronic Commerce Study. Singapore: APEC Publishing.

Arendt, L. (2008). Barriers to ICT adoption in SMEs: how to bridge the digital divide?. Journal of Systems and Information Technology, 10(2), 93-108. http://dx.doi.org/10.1108/13287260810897738

Bayo-Moriones, A., \& Lera-López, F. (2007). A firm-level analysis of determinants of ICT adoption in Spain. Technovation, 27(6), 352-366. http://dx.doi.org/10.1016/j.technovation.2007.01.003

Beatty, R.C., Shim, J., \& Jones, M.C. (2001). Factors influencing corporate web site adoption: A timebased assessment. Information \& management, 38(6), 337-354. http://dx.doi.org/10.1016/S03787206(00)00064-1 
Benham, H.C., \& Raymond, B.C. (1996). Information technology adoption: Evidence from a voice mail introduction. ACM SIGCPR Computer Personnel, 17(1), 3-25. http://dx.doi.org/10.1145/227005.227006

Blackwood, F. (1997). E-commerce benefits for small firms. San Francisco Business Times, 12(12), 25.

Brancheau, J.C., \& Wetherbe, J.C. (1990). The adoption of spreadsheet software: Testing innovation diffusion theory in the context of end-user computing. Information Systems Research, 1(2), 115-143. http://dx.doi.org/10.1287/isre.1.2.115

Cabarcas Álvarez, A., Martelo Gómez, R.J., \& Puello Marrugo, P. (2013). TICs en las Pymes: Herramienta útil para aumentar la competitividad. Ingeniator, 3(5), 10-17.

Cámara Colombiana de Informática y Tecnología \& Fedesarollo (2013). El papel de las TIC en el desarrollo de la pequeña empresa: reflexiones de política a la luz del caso colombiano. Retrieved from: Fedesarrollo. Centro de investigación económica y social website: http://www.fedesarrollo.org.co/wpcontent/uploads/2013/12/TIC diciembre 2013.pdf

Consoli, D. (2012). Literature analysis on determinant factors and the impact of ICT in SMEs. ProcediaSocial and Behavioral Sciences, 62, 93-97. http://dx.doi.org/10.1016/j.sbspro.2012.09.016

Cragg, P.B. (1990). Information technology and small firm performance. Loughborough University.

Cron, W.L., \& Sobol, M.G. (1983). The relationship between computerization and performance: A strategy for maximizing the economic benefits of computerization. Information \& management, 6(3), 171-181. http://dx.doi.org/10.1016/0378-7206(83)90034-4

Chapman, P., James-Moore, M., Szczygiel, M., \& Thompson, D. (2000). Building internet capabilities in SMEs. Logistics Information Management, 13(6), 353-361. http://dx.doi.org/10.1108/09576050010355662

Chaudhury, A., \& Bharati, P. (2008). IT Outsourcing Adoption by Small and Medium Enterprises: A Diffusion of Innovation Approach. Paper presented at the AMCIS 2008 Proceedings. Paper 390. Available online at: http://aisel.aisnet.org/amcis2008/390

Chesher, M., \& Skok, W. (2000). Roadmap for successful information technology transfer for small businesses. Paper presented at the Proceedings of the 2000 ACM SIGCPR conference on Computer personnel research. http://dx.doi.org/10.1145/333334.333338

Davis, F.D. (1989). Perceived usefulness, perceived ease of use, and user acceptance of information technology. MIS quarterly, 13(3), 319-340. http://dx.doi.org/10.2307/249008

ECTEL (2009). Use of ICT by SMEs in the ECTEL member states. Retrieved from ECTEL website: http://www.ectel.int/index.php/resources/publications 
Fillis, I., Johannson, U., \& Wagner, B. (2004). Factors impacting on e-business adoption and development in the smaller firm. International Journal of Entrepreneurial Behavior \& Research, 10(3), 178-191. http://dx.doi.org/10.1108/13552550410536762

Ghobakhloo, M., Arias-Aranda, D., \& Benitez-Amado, J. (2011). Adoption of e-commerce applications in SMEs. Industrial Management \& Data Systems, 111(8), 1238-1269. http://dx.doi.org/10.1108/02635571111170785

Gibbs, J.L., \& Kraemer, K.L. (2004). A cross-country investigation of the determinants of scope of ecommerce use: An institutional approach. Electronic Markets, 14(2), 124-137. http://dx.doi.org/10.1080/10196780410001675077

Grandon, E.E., \& Mykytyn Jr, P.P. (2004). Theory-based instrumentation to measure the intention to use electronic commerce in small and medium sized businesses. The Journal of Computer Information Systems, 44(3), 44-57. http://dx.doi.org/10.1016/j.im.2003.12.010

Grandon, E.E., \& Pearson, J.M. (2004). Electronic commerce adoption: an empirical study of small and medium US businesses. Information \& management, 42(1), 197-216.

Hair, J.F., Anderson, R.E., Tatham, R.L., \& Black, W.C. (1999). Análisis multivariante. Madrid: Prentice Hall.

Harindranath, G., Dyerson, R., \& Barnes, D. (2008). ICT in Small Firms: Factors Affecting the Adoption and Use of ICT in Southeast England SMEs. Paper presented at the ECIS 2008 Proceedings. Paper 167.

He, Q., Duan, Y., Fu, Z., \& Li, D. (2006). An innovation adoption study of online e-payment in Chinese companies. Journal of Electronic Commerce in Organizations, 4(1), 48-69. http://dx.doi.org/10.4018/jeco.2006010104

Hempel, P.S., \& Kwong, Y.K. (2001). B2B e-Commerce in emerging economies: i-metal. com's nonferrous metals exchange in China. Journal of Strategic Information Systems, 10(4), 335-355. http://dx.doi.org/10.1016/S0963-8687(01)00058-0

Hepworth, M., \& Ryan, J. (2000). Small firms in Europe's developing information society. In K. Ducatel, J. Webster \& W. Herrmann (Eds.), The Information Society in Europe. Work and Life in an Age of Globalization (pp. 73-96). Lanham, Md.: Rowman \& Littlefield Publishers.

Hsieh, C.-T., Jones, C., \& Lin, B. (2008). The new business potential with mobile commerce. International Journal of Mobile Communications, 6(4), 436-455. http://dx.doi.org/10.1504/IJMC.2008.018052 
Hussin, H., \& Noor, R.M. (2005). Innovating business through e-commerce: Exploring the willingness of Malaysian SMEs. Paper presented at the Proceedings of the Second International Conference on Innovation in IT.

Iacovou, C.L., Benbasat, I., \& Dexter, A.S. (1995). Electronic data interchange and small organizations: Adoption and impact of technology. MIS quarterly, 19(4), 465-485. http://dx.doi.org/10.2307/249629

ITU (2015). ICT Facts and Figures. Retrieved from: International Telecommunication Union website: http://www.itu.int/en/ITU-D/Statistics/Documents/facts/ICTFactsFigures2015.pdf

Ives, B., \& Olson, M.H. (1984). User involvement and MIS success: A review of research. Management science, 30(5), 586-603. http://dx.doi.org/10.1287/mnsc.30.5.586

Kendall, J.D., Tung, L.L., Chua, K.H., Ng, C.H.D., \& Tan, S.M. (2001). Receptivity of Singapore's SMEs to electronic commerce adoption. Journal of Strategic Information Systems, 10(3), 223-242. http://dx.doi.org/10.1016/S0963-8687(01)00048-8

Kumbakara, N. (2008). Managed IT services: The role of IT standards. Information Management \& Computer Security, 16(4), 336-359. http://dx.doi.org/10.1108/09685220810908778

Lee, J. (2004). Discriminant analysis of technology adoption behavior: A case of Internet technologies in small businesses. Journal of Computer Information Systems, 44(4), 57.

Lettieri, E., \& Masella, C. (2005). Measuring the value and sustainability of internet-based ICT in healthcare organisations. International journal of healthcare technology and management, 7(3-4), 319-332.

Limthongchai, P., \& Speece, M. (2003). The effect of perceived characteristics of innovation on ecommerce adoption by SMEs in Thailand. Paper presented at the Proceedings of the Seventh International Conference on Global Business and Economic Development, Bangkok, Thailand. Available online at: http://ssrn.com/abstract $=2565392$

Lin, H.-F., \& Lin, S.-M. (2008). Determinants of e-business diffusion: A test of the technology diffusion perspective. Technovation, 28(3), 135-145. http://dx.doi.org/10.1016/j.technovation.2007.10.003

Lucchetti, R., \& Sterlacchini, A. (2004). The adoption of ICT among SMEs: Evidence from an Italian survey. Small Business Economics, 23(2), 151-168. http://dx.doi.org/10.1023/B:SBEJ.0000027667.55821.53

Marston, S., Li, Z., Bandyopadhyay, S., Zhang, J., \& Ghalsasi, A. (2011). Cloud computing-The business perspective. Decision Support Systems, 51(1), 176-189. http://dx.doi.org/10.1016/j.dss.2010.12.006

Miller, M. (2008). Cloud computing: Web-based applications that change the way you work and collaborate online. Inidanapolis: QUE publishing. 
Ministerio de TIC (2014). Logros Plan Vive Digital. Available online at: http://micrositios.mintic.gov.co/vivedigital/logros-plan/index.php

Ministerio de TIC (2015). Boletín Trimestral de las TIC Cifras Cuarto Trimestre de 2014. Retrieved from: Colombia TIC Vive Digital website: http://colombiatic.mintic.gov.co/602/articles-8598_archivo_pdf.pdf

Mndzebele, N. (2013). The Effects of Relative Advantage, Compatibility and Complexity in the Adoption of EC in the Hotel Industry. International Journal of Computer and Communication Engineering, 2(4), 473-476. http://dx.doi.org/10.7763/IJCCE.2013.V2.229

Moghaddam, B.K., \& Khatoon-Abadi, A. (2013). Factors affecting ICT adoption among rural users: A case study of ICT Center in Iran. Telecommunications Policy, 37(11), 1083-1094. http://dx.doi.org/10.1016/j.telpol.2013.02.005

Molla, A., \& Licker, P.S. (2005). Perceived e-readiness factors in e-commerce adoption: An empirical investigation in a developing country. International Journal of Electronic Commerce, 10(1), 83-110.

Moore, G.C., \& Benbasat, I. (1991). Development of an instrument to measure the perceptions of adopting an information technology innovation. Information Systems Research, 2(3), 192-222. http://dx.doi.org/10.1287/isre.2.3.192

Mykytyn Jr, P.P., \& Harrison, D.A. (1993). The application of the theory of reasoned action to senior management and strategic information systems. Information Resources Management Journal, 6(2), 15. http://dx.doi.org/10.4018/irmj.1993040102

Narayanasamy, K., Santhapparaj, A., \& Eze, U.C. (2008). An empirical study of website adoption among Small and Medium Enterprises (SMEs) in Malaysia. Communications of the IBIMA, 2(28), 339-351.

Ntemana, T.J., \& Olatokun, W. (2012). Analyzing the Influence of Diffusion of Innovation Attributes on Lecturers' Attitude Towards Information and Communication Technologies. Human Technology, 8(2), 179-197. http://dx.doi.org/10.17011/ht/urn.201211203034

Nunnally, J.C., \& Bernstein, I.H. (1994). Psychometric Theory. New York: McGraw-Hill.

OECD (2002). Information Technology Outlook 2002. Paris: OECD. http://dx.doi.org/10.1787/it_outlook-2002en

Orduz, R. (2011). Internet en Colombia: Los cambios de una década (2000-2010). Retrieved from: Colombia Digital website: http://www.colombiadigital.net/opinion/columnistas/los-numeros-de-las-tic/item/643internet-en-colombia-los-cambios-de-una-d\%C3\%A9cada-2000-2010.html 
Parisot, A.H. (1995). Technology and teaching: The adoption and diffusion of technological innovations by a community college faculty. Montana State University, Bozeman, MT.

Premkumar, G. (2003). A meta-analysis of research on information technology implementation in small business. Journal of organizational computing and electronic commerce, 13(2), 91-121. http://dx.doi.org/10.1207/S15327744JOCE1302_2

Premkumar, G., \& King, W.R. (1994). Organizational characteristics and information systems planning: an empirical study. Information Systems Research, 5(2), 75-109. http://dx.doi.org/10.1287/isre.5.2.75

Premkumar, G., Ramamurthy, K., \& Nilakanta, S. (1994). Implementation of electronic data interchange: An innovation diffusion perspective. Journal of Management Information Systems, 11(2), 157-186. http://dx.doi.org/10.1080/07421222.1994.11518044

Ramdani, B., \& Kawalek, P. (2007). SMEs \& IS innovations adoption: a review \& assessment of previous research. Academia. Revista Latinoamericana de Administración, (39), 47-70.

Riemenschneider, C.K., \& Mckinney, V.R. (2001). Assessing belief differences in small business adopters and non-adopters of web-based e-commerce. Journal of Computer Information Systems, 42(2), 101.

Rogers, E.M. (1995). Diffusion of innovations (4 ed.). New York, NY: Free Press.

Rouibah, K., Khalil, O.E., \& Hassanien, A.E. (2009). Emerging Markets and E-Commerce in Developing Economies. Hershey, New York: Information Science Reference. http://dx.doi.org/10.4018/978-1-60566$100-1$

Saffu, K., Walker, J.H., \& Hinson, R. (2007). An empirical study of perceived strategic value and adoption constructs: The Ghanaian case. Management Decision, 45(7), 1083-1101. http://dx.doi.org/10.1108/00251740710773925

Sahin, I. (2006). Detailed Review of Rogers' Diffusion of Innovations Theory and Educational Technology-Related Studies Based on Rogers' Theory. Turkish Online Journal of Educational Technology, $5(2), 14-23$.

Scupola, A. (2001). Adoption of Internet-based electronic commerce in Southern Italian SMEs. Paper presented at the 1 st Nordic Workshop on Electronic Commerce, Halmstad, Sweden.

Slegers, C., Singh, S., \& Hall, J. (1998). Small business and electronic commerce: an Australian survey. Melbourne: CIRCIT at RMIT.

Sokolov, I.P. (2009). Cloud Computing: Overview, Concepts and Business Deployment Scenarios. Vienna 
Spanos, Y.E., Prastacos, G.P., \& Poulymenakou, A. (2002). The relationship between information and communication technologies adoption and management. Information \& management, 39(8), 659-675. http://dx.doi.org/10.1016/S0378-7206(01)00141-0

Syed, S.A., Khatibi, A., Ismail, H., \& Ahmad, S. (2005). Perceived benefits of e-commerce adoption in the electronic manufacturing companies in Malaysia. Journal of Social Sciences, 1(3), 188-193. http://dx.doi.org/10.3844/jssp.2005.188.193

Tan, K.S., Chong, S.C., Lin, B., \& Eze, U.C. (2009). Internet-based ICT adoption: Evidence from Malaysian SMEs. Industrial Management \& Data Systems, 109(2), 224-244. http://dx.doi.org/10.1108/02635570910930118

Tan, K.S., \& Eze, U.C. (2008). An empirical study of internet-based ICT adoption among Malaysian SMEs. Communications of the IBIMA, 1(1), 1-12.

Tan, M., \& Teo, T.S. (2000). Factors influencing the adoption of Internet banking. Journal of the Association for Information Systems, 1(1), 1-42.

Teo, T.S.H., \& Pok, S.H. (2003). Adoption of WAP-enabled mobile phones among Internet users. Omega, 31(6), 483-498. http://dx.doi.org/10.1016/j.omega.2003.08.005

Thong, J.Y., \& Yap, C.-S. (1995). CEO characteristics, organizational characteristics and information technology adoption in small businesses. Omega, 23(4), 429-442. http://dx.doi.org/10.1016/03050483(95)00017-I

Thong, J.Y., Yap, C.-S., \& Raman, K. (1994). Engagement of external expertise in information systems implementation. Journal of Management Information Systems, 11(2), 209-231. http://dx.doi.org/10.1080/07421222.1994.11518046

Thong, J.Y.L. (1999). An integrated model of information systems adoption in small businesses. Journal of Management Information Systems, 15(4), 187-214. http://dx.doi.org/10.1080/07421222.1999.11518227

Tiwana, A., \& Bush, A.A. (2007). A comparison of transaction cost, agency, and knowledge-based predictors of IT outsourcing decisions: A US-Japan cross-cultural field study. Journal of Management Information Systems, 24(1), 259-300. http://dx.doi.org/10.2753/MIS0742-1222240108

Tornatzky, L.G., Fleischer, M., \& Chakrabarti, A.K. (1990). The processes of technological innovation. Lexington: Lexington Books. 
Van Slyke, C., Belanger, F., \& Comunale, C.L. (2004). Factors influencing the adoption of web-based shopping: the impact of trust. ACM Sigmis Database, 35(2), 32-49.

http://dx.doi.org/10.1145/1007965.1007969

Venkatesh, V., Morris, M.G., Davis, G.B., \& Davis, F.D. (2003). User acceptance of information technology: Toward a unified view. MIS quarterly, 27(3), 425-478.

Wang, Y., Meister, D., \& Wang, Y. (2008). Relative Advantage and Perceived Usefulness: The Adoption of Competing ICT. Paper presented at the DIGIT 2008 Proceedings. Paper 6. Available online at: http://aisel.aisnet.org/digit2008/6

Weiss, J.A., \& Dale, B.C. (1998). Diffusing against mature technology: Issues and strategy. Industrial Marketing Management, 27(4), 293-304. http://dx.doi.org/10.1016/S0019-8501(97)00062-X

Wymer, S.A., \& Regan, E.A. (2005). Factors influencing e-commerce adoption and use by small and medium businesses. Electronic Markets, 15(4), 438-453. http://dx.doi.org/10.1080/10196780500303151

Yap, C.-S., Thong, J.Y.L., \& Raman, K.S. (1994). Effect of government incentives on computerisation in small business. European Journal of Information Systems, 3(3), 191-206.

http://dx.doi.org/10.1057/ejis.1994.20

Yap, C.S., \& Walsham, G. (1986). A survey of information technology in the UK service sector. Information \& management, 10(5), 267-274. http://dx.doi.org/10.1016/0378-7206(86)90029-7

Zhang, P., Aikman, S.N., \& Sun, H. (2008). Two types of attitudes in ICT acceptance and use. International Journal of Human-Computer Interaction, 24(7), 628-648.

http://dx.doi.org/10.1080/10447310802335482

Zhu, K., \& Kraemer, K.L. (2005). Post-adoption variations in usage and value of e-business by organizations: Cross-country evidence from the retail industry. Information Systems Research, 16(1), 61-84. http://dx.doi.org/10.1287/isre.1050.0045

Intangible Capital, 2016 (www.intangiblecapital.org)

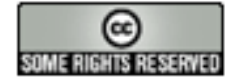

Article's contents are provided on an Attribution-Non Commercial 3.0 Creative commons license. Readers are allowed to copy, distribute and communicate article's contents, provided the author's and Intangible Capital's names are included. It must not be used for commercial purposes. To see the complete license contents, please visit http://creativecommons.org/licenses/by-nc/3.0/. 